NBER WORKING PAPER SERIES

\title{
SHOULD GERMANY HAVE BUILT A NEW WALL? MACROECONOMIC LESSONS FROM THE 2015-18 REFUGEE WAVE
}

\author{
Christopher Busch \\ Dirk Krueger \\ Alexander Ludwig \\ Irina Popova \\ Zainab Iftikhar \\ Working Paper 26973 \\ http://www.nber.org/papers/w26973
}

\author{
NATIONAL BUREAU OF ECONOMIC RESEARCH \\ 1050 Massachusetts Avenue \\ Cambridge, MA 02138 \\ April 2020
}

This paper is being prepared for the Carnegie-Rochester Conference on Migration Policy: Implications for Growth, Inequality, and Welfare. We thank our discussants Tommaso Porzio, Sebastian Heise and Chris Moser, as well as Friedrich Breyer, Alfred Garloff, Chiara Lacava, Joan Llull, Kjetil Storesletten and Marco Weißler and seminar participants at Houston, Montreal, Northwestern, UQAM, Wharton, the UPenn Macro Public Finance, EIEF and Carnegie Rochester NYU Conferences, the Norface TRISP Meeting and the Macro Inequality Group at UAB for very helpful comments and Anna-Maria Maurer for excellent research assistance. Chris Busch gratefully acknowledges financial support from the Spanish Ministry of Economy and Competitiveness, through the Severo Ochoa Programme for Centres of Excellence in R\&D. Alex Ludwig gratefully acknowledges financial support by NORFACE Dynamics of Inequality across the Life-Course (TRISP) grant 462-16-120 and by the Research Center SAFE, funded by the State of Hessen initiative for research LOEWE. Dirk Krueger thanks the NSF for support under grant SES 1757084. The views expressed herein are those of the authors and do not necessarily reflect the views of the National Bureau of Economic Research.

NBER working papers are circulated for discussion and comment purposes. They have not been peer-reviewed or been subject to the review by the NBER Board of Directors that accompanies official NBER publications.

(C) 2020 by Christopher Busch, Dirk Krueger, Alexander Ludwig, Irina Popova, and Zainab Iftikhar. All rights reserved. Short sections of text, not to exceed two paragraphs, may be quoted without explicit permission provided that full credit, including $\odot$ notice, is given to the source. 
Should Germany Have Built a New Wall? Macroeconomic Lessons from the 2015-18 Refugee

Wave

Christopher Busch, Dirk Krueger, Alexander Ludwig, Irina Popova, and Zainab Iftikhar

NBER Working Paper No. 26973

April 2020

JEL No. E20,F22,H55

\section{ABSTRACT}

In 2015-2016 Germany experienced a wave of predominantly low-skilled refugee immigration. We evaluate its macroeconomic and distributional effects using a quantitative overlapping generations model calibrated using German micro data to replicate education and productivity differentials between foreign born and native workers. Workers are modelled as imperfect substitutes in aggregate production leading to endogenous wage differentials. We simulate the dynamic effects of this refugee wave, with specific focus on the welfare impact on low skilled natives. Our results indicate that the small losses this group suffers can be compensated by welfare gains of other parts of the native population.

Christopher Busch

Universitat Autonoma de Barcelona,

MOVE, and Barcelona GSE

Campus de Bellaterra-UAB Edifici B

ES-08193 Cerdanyola del Vallès

Barcelona, Spain

chris.busch@movebarcelona.eu

Dirk Krueger

Economics Department

University of Pennsylvania

The Ronald O. Perelman Center

for Political Science and Economics

133 South 36th Street

Philadelphia, PA 19104

and NBER

dkrueger@econ.upenn.edu

Alexander Ludwig

Goethe University Frankfurt

House of Finance

Theodor-W.-Adorno-Platz 3

D-60629 Frankfurt am Main

Ludwig@safe.uni-frankfurt.de
Irina Popova

Goethe University Frankfurt

House of Finance

Theodor-W.-Adorno-Platz 3

D-60629 Frankfurt am Main

popova@econ.uni-frankfurt.de

Zainab Iftikhar

Goethe University Frankfurt

House of Finance

Theodor-W.-Adorno-Platz 3

Frankfurt 60629

Germany

iftikhar@econ.uni-frankfurt.de 


\section{Introduction}

In the last few years, Europe has experienced a massive refugee wave from Africa and Asia (and Syria specifically) that has brought in an inflow of mostly young, mostly unskilled refugees, substantially increasing the flow in-migration into Western Europe from other parts of the world. Germany has been the recipient of a large share of these refugees. In 2015 and 2016 alone, ca. 2 million political and economic migrants came to Germany, making it by far the largest recipient of migration in Europe. This flow is large in absolute terms, relative to a total population in Germany of 82 million, and has created substantial political backlash and the rise of right-wing political parties. The flow is comparable to the flow of individuals migrating from East to West Germany after World War II, inducing the political regime in East Germany to build the Berlin Wall in 1961. It is larger than the net inflow of ca. 1.8 million individuals from East Germany into West Germany from 1989 to 2006 after the wall came down in 1989, see Glorius (2010). This in-migration of young foreigners occurs against the backdrop of a secular massive ageing of the native German population, raising the possibility that reforms of the public pay-as-you-go pension system necessitated by population ageing could be postponed or moderated.

Motivated by these observations we evaluate the macroeconomic and welfare implications of the large wave of refugee inflows into the German economy in the short- and in the long run. Simply put, we ask whether it would have been in the economic interest of the local population to erect, figuratively speaking, a new wall to fence Germany off from the observed migration flow. The more nuanced question we answer is what are the economic characteristics of the group of natives that are most bound to gain and to lose from a large-scale refugee wave into a high-income ageing domestic economy with a mature social insurance system. We view Germany as an interesting case study with excellent micro data on migrants (which aids the calibration and estimation of our model) that was subject to a large migrant inflow in recent years, but the main economic forces, and thus the main results, we conjecture, apply to other recipient countries as well.

To answer our research question we employ a quantitative OLG model with time-varying demographic structure and neoclassical production that is subject to empirically realistic inflows of a low-skilled migrant population. To gain intuition we first construct a simple twoperiod version of the model as in Diamond (1965), and show analytically that these migrant inflows have four main impacts on welfare of natives. First, they raise overall labor supply and thus lower the capital-labor ratio and wages, and increase rates of return, as long as the economy is closed. Second, the migration inflow changes the supply of low-skilled foreign workers, relative to that of their native counterparts, and relative to high-skilled labor. 
If these workers are imperfect substitutes, then wages of skilled native workers rise (since skilled workers have become relatively scarcer), but the impact on wages of unskilled native workers is ambiguous. Unskilled workers are now more abundant, which lowers their wages. But to the extent that unskilled native and unskilled migrant workers are also imperfect substitutes, the native unskilled are now scarcer as well. The net effect on the relative wage of this group is then determined by the substitutability of skilled v/s unskilled labor relative to the substitutability between unskilled native and migrant labor. If this latter substitution elasticity is high relative to the former (as we estimate empirically), relative wages of native unskilled workers fall. Third, since migrants are young, an increase in their inflow reduces the old-age dependency ratio and increases the relative return on the PAYGO social security system for native contributors. Finally, the inflow of low-skilled migrants leads to an increase of tax-financed administrative government expenditures which reduces welfare of the natives.

We then extend our analysis to a quantitative OLG economy with a national labor market in order to quantify the relative importance of these four channels. As in the simple model workers with differential skill levels and different migratory background are imperfect substitutes in production. We model the public social security system closely following the actual German system, and introduce a realistic demographic structure, including a demographic transition towards an ageing population. This demographic transition necessitates reforms of the social security system in the absence of migration inflows of young workers. Our main thought experiment consists of a sudden, unexpected inflow of refugees of the size and composition experienced in the years 2015 to 2018. We compute the transition induced by this refugee wave and contrast it with the scenario in which the refugee inflow does not occur, and thus the ageing of the population continues at pre-refugee speed. By comparing these scenarios we quantify the macroeconomic, distributional and welfare consequences for natives in different skill classes from this recent refugee wave in the short- and long run.

In order to conduct our quantitative analysis we require as inputs aggregate migration flows, the skill composition of migrants, as well as micro estimates for wage profiles and assimilation speeds of migrants. To derive the latter two we turn to micro data from the German Socio-Economic Panel Study (SOEP). The structure of the SOEP allows us to measure wages of immigrants from different geographic origins over a long period of time (1984-2017). We use this information to estimate the elasticity of substitution between different groups of natives and immigrants, key ingredients in the aggregate production function for the quantitative analysis. Apart from the core samples of the SOEP we also use data from the IAB-SOEP Migration Sample (2013-2017), which oversamples immigrants from Arab and Islamic countries, the main source countries of the immigration wave from 2015-18 we study, as well as the IAB-BAMF-SOEP Refugee sample (2015-2017), which 
samples the refugee population that arrived in Germany in the years of interest. We use this information to characterize the incumbent migrant population and the incoming refugees.

Figure 1: Wages since Immigration

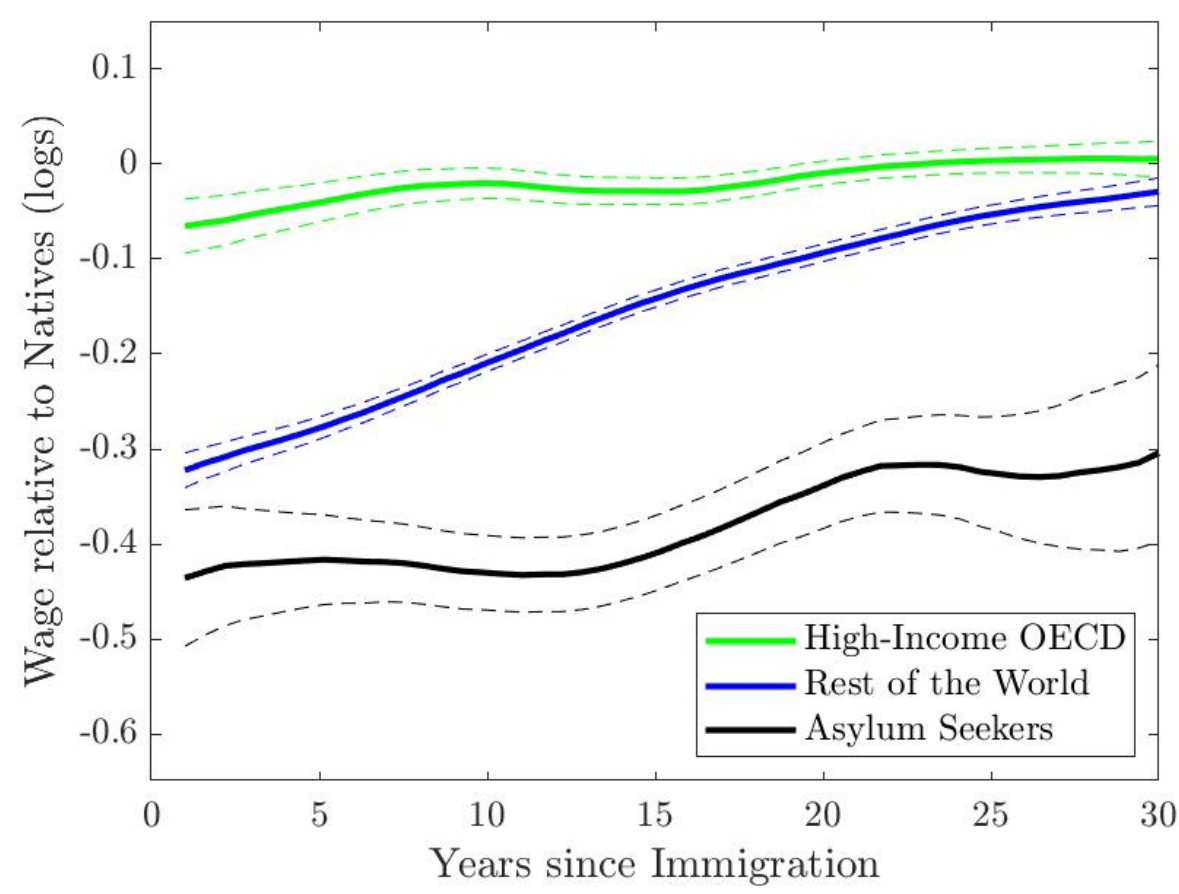

Note: Wages of migrants, relative to natives with the same level of education, age and family background, as a function of the time since arrival in Germany. Source: Own calculations based on SOEP.

Figure 1 displays wages of migrants, relative to natives with the same level of education, age and family background, as a function of the time since arrival in Germany. It shows very sizeable initial wage discounts, in the order of $30 \%$ for economic immigrants from poor countries, and in excess of $40 \%$ for political asylum seekers, with very significant convergence towards native German wages over time, especially for the economic migrants, but at much slower speed for political refugees. ${ }^{1}$ As documented in Brell, Dustmann, and Preston (2020), labor market participation rates of asylum seekers are initially low and increase slowly over time. In our evaluation of the economic impact of the low-skill in-migration to Germany low wages, low participation rates and slow convergence are key data moments targeted in the quantitative analysis. The availability of rich micro data on migrant labor market outcomes is an important benefit of quantitatively analyzing the German experience in the context of world-wide immigration flows in the last five years. Equipped with these key

\footnotetext{
${ }^{1}$ The much slower convergence among the asylum seekers might not be due to slow productivity convergence, but also reflects restricted access to the German labor market by this group stemming from administrative hurdles described in section 3. It could also stem from reduced incentives to accumulate Germany-specific human capital since political refugees face a much larger probability of return migration.
} 
empirical ingredients we simulate the macroeconomic and distributional impacts of the recent migration wave. We feed into our model the actual evolution of the domestic and migrant population (the high migration scenario), as well as two counterfactual scenarios in which we either fix migration flows to relatively low average levels (the baseline scenario) or only let political refugees in-migrate (the refugee migration scenario). We obtain three main quantitative findings. First, gross wages of unskilled natives deteriorate, on account of increased competition on the labor market from equally unskilled refugees. This effect is only partially offset by lower effective contributions to social security; thus net wages of low skilled natives decrease throughout our entire projection period. Thus, low skilled individuals currently alive experience welfare losses. Second, medium and high skilled natives experience significant welfare gains. Third, the aggregate gains, measured as consumption equivalent variation, are larger than the aggregate losses. This suggests that a compensation of low skilled natives is possible. Our aggregate welfare measure shows that the overall effects are positive, small initially, and increasing over time. Importantly, this aggregate measure masks the fact that the migration impact is very heterogeneous across the skill distribution and sizable for some population groups. Specifically, we find that the welfare losses of a young low-skilled native in the refugee migration scenario of age 35 in 2013 are about $-0.2 \%$ in terms of a consumption equivalent variation. This contrasts with welfare gains accruing to young high and middle-skilled natives also of $0.2 \%$.

Based on these results our answer to the question motivating this paper is that Germany should not have built a new wall. This is true even from the perspective of the native low-skilled population, which, among the natives, is most severely subjected to migrant competition on the German labor market, but only if this population group is compensated for their net wage losses using the gains of the rest of the German population.

\section{$2 \quad$ Related Literature}

Our work relates to four strands of the literature. First, we build on a large literature studying the effects of immigration on incomes and welfare of natives. An early influential paper is Borjas (1999) who shows that an influx of immigrants into a host country (the U.S. in his context) leads to redistribution among natives towards capital income and an overall net benefit to native households which Borjas calls the 'Immigration Surplus'. As long as capital and (immigrant) labor are not perfect substitutes in production. Ben-Gad (2004) extends the analysis to endogenous labor supply and capital accumulation and finds a smaller immigration surplus to the U.S. The same effect of an inflow of labor will be present in our analysis, and it dampens the long-run decline in the labor force due to the demographic 
transition of the native population. Motivated by the evidence in Borjas (2003) subsequent papers assume imperfect substitution among workers with different characteristics so that immigration lowers the wages of competing workers. Ben-Gad (2008) allows for heterogeneous skills and concludes the immigration surplus is maximized with skilled immigration due to complementarity between skilled labor and capital. In a study for Germany D'Amuri et al. (2010) show that the pre-2010 inflow of immigrants leaves wages and employment levels of natives broadly unaffected; incumbent immigrants are adversely affected by the inflow of new migrants. Felbermayr et al. (2010) and Ottaviano and Peri (2012) largely confirm these conclusions. In our model we take these relative gross wage responses into account by allowing for a flexible substitution structure across skill and nationality groups. This approach is subject to the criticism by Dustmann et al. (2016) who argue that the assumption that migrants can be sorted into conventional experience-, education- and nationality cells might be invalid, a concern we address through sensitivity analyses. ${ }^{2}$

Second, our paper is related to the literature on assimilation of economic migrants and political refugees. Borjas (1985) emphasizes the (positive) selection problem of immigrants who stay in a given country when estimating assimilation speeds. More recently, the work by Weiss et al. (2003) and Eckstein and Weiss (2004) study the adjustment of occupational choices and human capital formation for wage growth of immigrants to Israel. Dustmann and Preston (2012) point to initial skill degradation among immigrants upon arrival into host countries and Lessem and Sanders (2019) find skill upgrading of immigrants over time, emphasizing the role played by language skill in this process. ${ }^{3}$ Finally, Brell et al. (2020) discuss the differences in the labor market integration between economic immigrants and political refugees. Motivated by this evidence we capture assimilation by allowing the conditional productivity of immigrants to increase with time since immigration, by estimating assimilation speeds of wage processes, based on the data shown in Figure 1. Consistent with Brell et al. (2020) we allow these speeds to differ between economic and political migrants.

Third, regarding the fiscal effects of migration we follow Storesletten (2000), who analyzes the impact of immigration to the U.S. from 1960-1990 in a general equilibrium overlapping generation economy such as ours. The key finding is that only medium- and high-skilled immigration can ease the fiscal burden in the country, whereas low-skilled immigration cannot. In the context of the ageing population of Japan, Imrohoroglu et al. (2017) reach similar

\footnotetext{
${ }^{2}$ Llull (2018) observes that imperfect substitutability across workers is an endogenous outcome of heterogeneous education, labor supply and career choices of natives and incumbent immigrants to show that natives who are close competitors of immigrants are adversely affected. Similarly, Colas (2019) argues that natives and old immigrants respond to new immigrants by internally migrating to different labor markets, and Burstein et al. (2017) analyze how these adjustments vary across tradeable and non-tradeable sectors.

${ }^{3}$ Lagakos et al. (2018) show that returns to experience in the birth country before migrating to the U.S. are positively correlated with birth-country GDP per capita.
} 
conclusions. Chojnicki et al. (2011) perform a retrospective analysis of the immigration wave of 1945-2000 to the U.S. and conclude that welfare gains could have been achieved for natives had the past immigration to the U.S. been dominantly high-skilled. Similarly, the findings of Guerreiro et al. (2019) suggest that free immigration is welfare-maximizing for natives if immigrants can be excluded from the social welfare system. Finally, using SOEP data Kirdar (2012) estimates the fiscal impact of immigration on Germany in the presence of an endogenous return migration choice. ${ }^{4}$

Finally, this paper contributes to studies on the impact of the most recent immigration wave into the European Union, and into Germany specifically. d'Artis Kancs and Lecca (2018) analyze alternative refugee integration scenarios for the period 2016-2040 to show that full repayment of investment in refugees' integration is achieved in 9 to 19 years and that immigration has a positive growth effect on the German economy. ${ }^{5}$ Scharfbillig and Weissler (2019) find no evidence that immigrants displace employment of natives but that employment of incumbent migrants is adversely affected. Their results suggest that natives and immigrants are imperfect substitutes in production despite having similar qualifications, whereas the degree of substitution between asylum seekers and other migrant residents of Germany is higher, which is a key ingredient in our quantitative model.

\section{Institutional Background}

In this section we give a brief description of the historical and institutional background of the large migration inflow in 2015-16. The goal is not to provide a comprehensive review, but rather give the background for justifying key modelling assumptions in the quantitative model. More than 50\% of the increase in migrants in 2015 and 2016 stems from political refugees originating in Syria. The Syrian civil war officially began on March 15, 2011. Political asylum seekers started arriving in numbers via land and sea in Europe in 2013, and in 2015 the crisis reached its peak when the EU received more than one million asylum applications from Syria alone. Germany was the main destination country for these refugees. Chancellor Angela Merkel announced in August 2015 a temporarily suspension of the EU Dublin regulations which required refugees to apply for asylum in the country to which they arrive first. In September 2015 Germany agreed to let refugees from Hungary enter Germany.

As the flow of new asylum seekers subsided in 2017, the focus of policy shifted towards the integration of these refugees into the German labor market. In August, 2016 the Pri-

\footnotetext{
${ }^{4}$ A related recent literature uses frictional labor market models and stresses positive effects on immigration on host economies through endogenous job creation, see e.g. Chassamboulli and Palivos (2014), Nanos and Schluter (2014), Moreno-Galbis and Tritah (2016), Battisti et al. (2017) and Iftikhar and Zaharieva (2019).

${ }^{5}$ Related, Stähler (2017) studies the macroeconomic effects of (failed) integration.
} 
ority Review policy that restricted the access of the immigrants to the labor markets was suspended until August of 2019. In June 2019 "The Alien Employment Promotion Act" was adopted to promote assistance with asylum procedures and integration into the labor market. Whereas officially recognized political refugees (those having been granted asylum) have unrestricted access to German job market and have the same rights as German citizens, asylum seekers cannot access the labor market during the first three months of their arrival in Germany. After this waiting period is over access to the labor market is granted with restrictions. In order to get a work permit the asylum seeker must have a job offer and the German job centers examine that neither an EU citizen nor a non-EU citizen with a residence permit is displaced as a result of hiring the asylum seeker. All asylum seekers are barred from taking up self-employment for the duration of their asylum procedure. These restrictions negatively impact employment opportunities of asylum seekers and their wages. Consequently, wages and earnings of these immigrants are initially low, as Figure 1 showed.

Finally, in order to evaluate the fiscal consequences of the recent migration wave, it is important to assess the extent to which migrants qualify for social assistance. Asylum Seekers are provided social and medical benefits in accordance with the Asylum-Seekers' Benefits Act. Benefits include food, housing, heating, healthcare, personal hygiene, assistance in sickness, pregnancy and birth as well as household durables and consumables. In October, 2015 the level of social benefits were raised and 'in kind' benefits were substituted by 'in cash' benefits. Furthermore asylum seekers are entitled to standard social benefits and full healthcare after receiving social benefits under Asylum Seekers' Benefits Act for 15 months. Thus, it is a fairly accurate approximation of reality to assume that asylum seekers are eligible for the same type of social assistance payments as natives, certainly after an initial

period in which these benefits are moderately lower. For a refugee (i.e. a successful asylum applicant) the same statement applies.

Against this background, and motivated by the massive inflow of migrants in 2015-16 into an aging German labor market, we first develop a simple model to study the qualitative impact of these developments, before quantifying them in a more realistic version intended to capture the institutional details of Germany in this period more accurately.

\section{Simple Model}

We now develop an OLG model with two-period lived households whose basic structure will also form the foundation for the quantitative model in the next section. The purpose of this simple model is to clarify the main trade-offs from the recent migration inflows. On one hand, the asylum seeking immigrants are on average young, and thus help to stabilize 
social security budgets. On the other hand, at least initially these migrants have low labor productivity in the German labor market and the migration system has to be administered which is costly. In addition, migrants impact wages of natives in the labor market.

Competitive firms operate a technology that uses capital and three types of labor. There are high-skilled $(h i)$ native $(n a)$ workers and low skilled workers $(l o)$, which might either also be natives or foreigners $\left(f_{o}\right)$. These three different groups of workers are assumed to be imperfect substitutes in production. This structure captures three main effects of an inflow of migrants on gross wages: First, it increases the relative scarcity of high skilled workers, which, in a model with imperfect substitutability, increases relative wages of the high skilled and reduces wages of low-skilled workers. Second, if low-skilled natives and low-skilled foreigners are imperfect substitutes, an increase in migration raises the relative wage of low-skilled natives. Finally, an inflow of workers increases overall labor supply, and thus decreases the wage level in general equilibrium.

We assume that the economy is ageing, modelled as an exogenous population growth rate $\gamma^{n}<1$ of the native population. In addition, in every period a number of young migrants enter the country, shifting the demographic composition of the population towards younger individuals. In retirement, households earn social security income, which is related to past contributions in a Bismarckian pay-as-you-go (PAYGO) pension scheme. Pensions in the PAYGO system are financed by levying the contribution rate $\tau$ on labor income of young workers. Migration increases the population growth rate and thus the share of the young in the population, thereby raising the implicit rate of return of the pension system.

\subsection{Population}

There are two-period lived households. We denote by $N_{t}(0)$ the size of the period $t$ young population and by $N_{t}(1)$ the size of the old. We ignore mortality risk, thus $N_{t+1}(1)=N_{t}(0)$. The young population in each period $t$ consists of native high skill workers, $N_{t}(0, h i, n a)$, and low skill native- and foreign-born workers $N_{t}\left(0, l_{o}\right)=N_{t}(0, l o, n a)+N_{t}\left(0, l_{o}, f o\right)$. There is a constant share $\omega$ of high skilled workers in the native population, thus $N_{t}(0, h i, n a)=$ $\omega N_{t}(0, n a)$ and $N_{t}(0, l o, n a)=(1-\omega) N_{t}(0, n a)$. The population grows at an exogenous rate $\gamma_{t}$ and thus the young population in $t$ is given by $N_{t}(0)=\gamma_{t} N_{t-1}(0)$.

This population growth rate $\gamma_{t}$ is determined jointly by the fertility rate of the native population and the migration rate of individuals from abroad. Let $\gamma_{t}^{n}$ denote the birth rate of the native population. We assume that once migrants enter the country, they have the same fertility rate as native individuals. We further express the exogenous migration flow as a share of the population stock of the young population in the previous period, 
$N_{t}(0, l o, f o)=\mu_{t} N_{t-1}(0)$. Combining these two assumptions yields

$$
N_{t}(0)=\gamma_{t}^{n} N_{t-1}(0)+N_{t}(0, l o, f o)=\left(\gamma_{t}^{n}+\mu_{t}\right) N_{t-1}(0)=\gamma_{t} N_{t-1}(0)
$$

Therefore the population growth rate $\gamma_{t}=\gamma_{t}^{n}+\mu_{t}$ is the sum of the fertility rate $\gamma_{t}^{n}$ of individuals living in the country when young, and the migration rate $\mu_{t}$. A positive immigration $\mu_{t}>0$ then acts like an increase in the fertility rate of the economy.

\subsection{Technology}

Production takes place with a nested CES-Cobb-Douglas production function of form

$$
\begin{aligned}
Y_{t} & =K_{t}^{\alpha} L_{t}^{1-\alpha} \\
L_{t} & =\left(L_{t}(l o)^{1-\frac{1}{\sigma_{l h}}}+L_{t}(h i)^{1-\frac{1}{\sigma_{l h}}}\right)^{\frac{1}{1-\frac{1}{\sigma_{l h}}}} . \\
L_{t}(l o) & =\left(L_{t}(l o, n a)^{1-\frac{1}{\sigma_{n f}}}+L_{t}(l o, f o)^{1-\frac{1}{\sigma_{n f}}}\right)^{\frac{1}{1-\frac{1}{\sigma_{n f}}}} .
\end{aligned}
$$

Here $\sigma_{l h}$ is the elasticity of substitution between skilled and unskilled labor, and $\sigma_{n f}$ is the elasticity of substitution between low-skilled labor supplied by natives and migrants. Notice that $L_{t}(s, i)$ are efficiency weighted units of labor with productivity differences across types of workers described next. Finally, there is full depreciation of capital, $\delta=1$.

\subsection{Households}

Households work one unit of time when young and have skill-specific $(s \in\{l o, h i\})$ labor productivity $\epsilon(s, i)$ that also depends on their migratory origin $i \in\left\{n a, f_{o}\right\}$. We normalize $\epsilon(h i, n a)=1$. The group-specific wage per unit of time is denoted by $w_{t}(s, i)$, on which individuals pay social security contributions at rate $\tau_{t}$. When young they consume $c_{t}(0, s, i)$ and save $a_{t+1}(s, i)$. Assets earn a gross risk-free interest rate $R_{t+1}$. When old they receive retirement income $b_{t+1}(s, i)$ and consume $c_{t+1}(1, s, i)$. The budget constraints in the two periods of life for workers in group $s, i$, are thus given by

$$
\begin{gathered}
c_{t}(0, s, i)+a_{t+1}(s, i)=w_{t}(s, i)\left(1-\tau_{t}\right) \\
c_{t+1}(1, s, i)=a_{t+1}(s, i) R_{t+1}+b_{t+1}(s, i) .
\end{gathered}
$$


Households born in period $t$ of type $(s, i)$ have logarithmic preferences over consumption, discount the future with factor $\beta$. Thus, their lifetime utility function $U_{t}(s, i)$ given by

$$
U_{t}(s, i)=\ln \left(c_{t}(0, s, i)\right)+\beta \ln \left(c_{t+1}(1, s, i)\right)
$$

\subsection{Government}

The government organizes a PAYG pension system. We assume that pensions of workers of group $i$ are proportional to their wages when young, where the replacement rate $\rho_{t}$ determines the size of the social security system, and is assumed to be constant across all groups:

$$
b_{t}(s, i)=\rho_{t} \tau_{t-1} w_{t-1}(s, i) .
$$

The pension system is Bismarckian in that benefits are tied to past contributions, an accurate approximation of the actual German system. ${ }^{6}$ Note that $\rho_{t}$ can be interpreted as the internal gross return of the pension system since (7) implies

$$
\rho_{t}=\frac{b_{t}(s, i)}{\tau_{t-1} w_{t-1}(s, i)}
$$

In addition, the government has to finance the bureaucracy in charge of integrating migrants into Germany. We assume that per migrant a resource cost of $\tilde{\kappa}_{t} \tau_{t} w_{t}$ is required to administer the migration system. These resource costs constitute lost output rather than transfers, and for analytical convenience we express them as a share $\tilde{\kappa}_{t}$ of tax payments. The total cost for migrants then depends on the number of migrants, and is given by

$$
\tilde{\kappa}_{t} \tau_{t} w_{t} N_{t}\left(0, l o, f_{o}\right)=\kappa_{t} \tau_{t} w_{t} L_{t}
$$

where $\kappa_{t}=\tilde{\kappa}_{t} \frac{N_{t}(0, l o, f o)}{L_{t}}$. Written in this way, the cost $\kappa_{t}$ captures both the cost per migrant $\tilde{\kappa}_{t}$, a parameter of the model, and the effect of an increase in the number of migrants (as parameterized by $\left.\mu_{t}\right)$, since the ratio $\frac{N_{t}(0, l o, f o)}{L_{t}}$ is strictly increasing in $\mu_{t}$.

Finally, we assume that the government budget is balanced in every period, which requires that the sequence of payroll tax rates $\left\{\tau_{t}\right\}$ satisfies:

$$
\tau_{t} \sum_{s, i} w_{t}(s, i) N_{t}(0, s, i)=\rho_{t} \tau_{t-1} \sum_{s, i} w_{t-1}(s, i) N_{t}(1, s, i)+\kappa_{t} \tau_{t} w_{t} L_{t} .
$$

\footnotetext{
${ }^{6}$ The German legislation does not feature dependency on $\tau_{t-1}$, but this is just a rescaling of the replacement rate because we could equivalently write $b_{t}(s, i)=\tilde{\rho}_{t} w_{t-1}(s, i)$ with $\tilde{\rho}_{t}=\rho_{t} \tau_{t-1}$.
} 
By assumption there are no high-skilled migrants in the model, and thus $N_{t}(0, h i, f o)=$ $N_{t}\left(1, h i, f_{o}\right)=0$. Given labor income taxes $\left\{\tau_{t}\right\}$ and costs for administrating migration (as parameterized by $\kappa_{t}$ ), the social security replacement rate $\rho_{t}$ adjusts to changes in the demographic composition of the population, to ensure government budget balance. ${ }^{7}$

\subsection{Characterization of Equilibrium}

We relegate a formal definition of the competitive equilibrium to appendix A. The key variable describing the dynamics of the competitive equilibrium is the capital-labor ratio $k_{t}=$ $\frac{K_{t}}{L_{t}}$. Starting from an initial capital $K_{0}$ and for an exogenous sequence of skilled and unskilled labor determined by fertility and migration rates as well as exogenous policy (social security replacement rates), once the dynamics of the capital-labor ratio is determined, factor prices, relative wages of each group, factor demands and consumption allocations of all private households follow directly from the firm's optimality conditions and the household budget constraints. The dynamics of the capital labor ratio itself is determined by private household savings decisions and asset market clearing.

We first characterize wages for a given sequence of the capital-labor-ratio, then demonstrating that in the model all households optimally choose the same saving rate, which determines the law of motion of the capital-labor ratio. We finally provide comparative statics with respect to the size of migration flow determined by $\mu_{t}$.

\subsubsection{Firm Optimization and Equilibrium Wages}

The representative firm hires three types of labor $L_{t}(h i), L_{t}(l o, n a), L_{t}\left(l o, f_{o}\right)$, combines them into a labor composite $L_{t}$ and uses $L_{t}$ and capital $K_{t}$ to produce output. Profit maximization implies that the gross return on capital and the wage per unit of the labor composite equal the marginal products of capital and labor, respectively:

$$
\begin{aligned}
1+r_{t} & =R_{t}=\alpha k_{t}^{\alpha-1} \\
w_{t} & =(1-\alpha) k_{t}^{\alpha}
\end{aligned}
$$

Furthermore, the wage per unit of time for a worker of type $(s, i)$ is determined by the product of its labor efficiency units $\epsilon(s, i)$, the wage per efficiency unit of the labor composite $w_{t}$ and

\footnotetext{
${ }^{7}$ For analytical convenience, in the simple model we consolidate the social security and general government revenue budget; the quantitative model will separate these two budgets, as is realistic for the German case.
} 
the marginal product of labor of type $(s, i)$ in producing the labor composite $L_{t}$, that is,

$$
w_{t}(s, i)=w_{t} \cdot \epsilon(s, i) \cdot \frac{\partial L_{t}}{\partial L_{t}(s, i)}
$$

Exploiting equations (3) and (4) yields wages (and thus labor incomes) as functions of the common wage per labor efficiency units as well as the relative scarcity of different demographic groups, whose impact is controlled by the substitution elasticities between skilled and unskilled labor $\sigma_{l h}$ and between unskilled labor of natives and migrants $\sigma_{n f}$.

$$
\begin{aligned}
w_{t}(h i) & =w_{t} \cdot\left(\frac{L_{t}}{L_{t}(h i)}\right)^{\frac{1}{\sigma_{l h}}} \\
w_{t}(l o, i) & =w_{t} \cdot \epsilon(l o, i) \cdot\left(\frac{L_{t}}{L_{t}(l o)}\right)^{\frac{1}{\sigma_{l h}}} \cdot\left(\frac{L_{t}(l o)}{L_{t}(l o, i)}\right)^{\frac{1}{\sigma_{n f}}}
\end{aligned}
$$

Exploiting the market clearing conditions (49) and (50) and the demographic relationships to express labor efficiency units in terms of demographic variables gives:

Proposition 1. Equilibrium wages of the different groups are determined as

$$
\begin{aligned}
w_{t}(h i) & =w_{t} \mathcal{W}_{h i}\left(\mu_{t} / \gamma_{t}^{n}\right) \\
w_{t}(l o, n a) & =w_{t} \mathcal{W}_{l o}\left(\mu_{t} / \gamma_{t}^{n}\right) \cdot \mathcal{W}_{n a}\left(\mu_{t} / \gamma_{t}^{n}\right)
\end{aligned}
$$

where the exogenous demographic factors $\mathcal{W}_{h i}\left(\mu_{t} / \gamma_{t}^{n}\right), \mathcal{W}_{n a}\left(\mu_{t} / \gamma_{t}^{n}\right)$ are increasing in $\mu_{t} / \gamma_{t}^{n}$ and $\mathcal{W}_{l o}\left(\mu_{t} / \gamma_{t}^{n}\right)$ is decreasing in $\mu_{t} / \gamma_{t}^{n}$. The wage $w_{t}$ per labor efficiency unit is a strictly increasing function purely of the aggregate capital-labor ratio $k_{t}$.

\subsubsection{Household Optimization}

To derive the equilibrium dynamics of the capital-labor ratio it is useful to restate the household maximization problem in terms of household saving rates

$$
s_{t}(s, i)=\frac{a_{t+1}(s, i)}{w_{t}(s, i)} .
$$

Using this definition and the household budget constraints we can rewrite lifetime utility as

$$
\begin{aligned}
U_{t}(s, i)= & (1+\beta) \ln \left(w_{t}\right)+\beta \ln \left(R_{t+1}\right)+(1+\beta) \ln \left(\frac{w_{t}(s, i)}{w_{t}}\right) \\
& +\ln \left(\left(1-\tau_{t}\right)-s_{t}(s, i)\right)+\beta \ln \left(s_{t}(s, i)+\frac{\rho_{t+1} \tau_{t}}{R_{t+1}}\right) .
\end{aligned}
$$


This expression clarifies the three forces impacted by population ageing and migration. First, demographic changes affect aggregate factor prices $\left(w_{t}, R_{t+1}\right)$ unless we analyze a small open economy. Second, it changes relative wages of the different population groups, as summarized by proposition 1 . Third, it changes the relative return on the social security system measured by $\frac{\rho_{t+1}}{R_{t+1}}$, and with it, the optimal saving decisions of households.

Taking first order conditions with respect to (17) gives the optimal saving rate as

$$
s_{t}(s, i)=\frac{\beta\left(1-\tau_{t}\right)-\frac{\rho_{t+1}}{R_{t+1}} \tau_{t}}{1+\beta}
$$

Note that the saving rate is identical across all population groups and only depends on the fiscal side of the model characterizing the PAYGO social security system. The only remaining endogenous variable in the saving rate and thus in the welfare of a given generation is the relative return of the social security system $\frac{\rho_{t+1}}{R_{t+1}}$. Using the budget constraint of the government (10) and noting that $N_{t+1}(i, 1)=N_{t}(i, 0)$ we have

$$
\begin{aligned}
\frac{\rho_{t+1}}{R_{t+1}} & =\frac{\tau_{t+1}}{\tau_{t} R_{t+1}} \frac{\sum_{s, i} w_{t+1}(s, i) N_{t+1}(s, i, 0)-\kappa_{t+1} w_{t+1} L_{t+1}}{\sum_{s, i} w_{t}(s, i) N_{t}(s, i, 0)} \\
& =\frac{\tau_{t+1}}{\tau_{t} R_{t+1}} \frac{\left(1-\kappa_{t+1}\right) w_{t+1} L_{t+1}}{w_{t} L_{t}}=\frac{\left(1-\kappa_{t+1}\right) \tau_{t+1}}{\tau_{t}} \frac{w_{t+1}}{R_{t+1} w_{t}} \gamma_{t+1}^{L}
\end{aligned}
$$

where $\gamma_{t+1}^{L}=\frac{L_{t+1}}{L_{t}}$ is the growth rate of aggregate labor supply in efficiency units, a function purely of the exogenous demographics of the model, as lemma 1 below shows. The general equilibrium term $\frac{w_{t+1}}{R_{t+1} w_{t}}$ is still endogenous and depends on the dynamics of the capital-labor ratio. To establish a benchmark we first characterize the saving rate and welfare in a small open economy where the interest rate $R$ is constant and exogenous, which, from the firm optimality conditions, implies a constant exogenous wage $w_{t}=w$ per labor efficiency unit and a constant exogenous capital-labor ratio.

\subsubsection{The Savings Rate and Welfare in a Small Open Economy}

With an exogenous interest rate $R$ the term $\frac{w_{t+1}}{R_{t+1} w_{t}}$ in equation (19) is exogenous and equals $\frac{w_{t+1}}{R_{t+1} w_{t}}=\frac{1}{R}$. The following proposition immediately follows from equations (18) and (17): 
Proposition 2. In a small open economy, the equilibrium saving rate and welfare of an individual of type $(s, i)$ born at time $t$ are given by

$$
\begin{aligned}
s_{t}(s, i) & =\frac{\beta\left(1-\tau_{t}\right)-\left(1-\kappa_{t+1}\right) \tau_{t+1} \frac{\gamma_{t+1}^{L}}{R}}{1+\beta}=s_{t} \\
U_{t}(s, i) & =(1+\beta) \ln (w)+\beta \ln (R)+(1+\beta) \ln \left(\frac{w_{t}(s, i)}{w_{t}}\right) \\
& +\beta \ln (\beta)-(1+\beta) \ln (1+\beta)+(1+\beta) \ln \left(1-\tau_{t}+\left(1-\kappa_{t+1}\right) \tau_{t+1} \frac{\gamma_{t+1}^{L}}{R}\right)
\end{aligned}
$$

\subsubsection{The Dynamics of the Capital-Labor Ratio in General Equilibrium}

In general equilibrium the ratio $\frac{w_{t+1}}{R_{t+1} w_{t}}$ is endogenous and determined by the dynamics of the capital-labor ratio. The market-clearing condition on the capital market implies

$$
K_{t+1}=\sum_{s, i} a_{t+1}(s, i) N_{t}(0, s, i)=\sum_{s, i} s_{t}(s, i) w_{t}(s, i) N_{t}(0, s, i)=s_{t} w_{t} L_{t}
$$

and thus

$$
\begin{aligned}
\frac{K_{t+1}}{L_{t+1}} & =k_{t+1}=s_{t} \frac{(1-\alpha) k_{t}^{\alpha}}{\gamma_{t+1}^{L}} \\
\frac{w_{t+1}}{R_{t+1} w_{t}} & =\frac{(1-\alpha) k_{t+1}^{\alpha}}{\alpha k_{t+1}^{\alpha-1}(1-\alpha) k_{t}^{\alpha}}=\frac{(1-\alpha) s_{t}}{\alpha \gamma_{t+1}^{L}} \\
\frac{\rho_{t+1}}{R_{t+1}} \tau_{t} & =\frac{\tau_{t+1}}{\tau_{t}} \frac{\left(1-\kappa_{t+1}\right) w_{t+1}}{R_{t+1} w_{t}} \gamma_{t+1}^{L} \tau_{t}=\frac{\left(1-\kappa_{t+1}\right) \tau_{t+1}(1-\alpha) s_{t}}{\alpha}
\end{aligned}
$$

Equations (18) and (26) can be solved for the saving rate in general equilibrium, which in turn determines general equilibrium welfare. These results are summarized in the following

Proposition 3. The general equilibrium saving rate and welfare of an individual of type $(s, i)$ born at time $t$ are given by

$$
\begin{aligned}
s_{t}(s, i) & =s_{t}=\frac{\alpha \beta\left(1-\tau_{t}\right)}{\alpha(1+\beta)+(1-\alpha)\left(1-\kappa_{t+1}\right) \tau_{t+1}} \\
U_{t}(s, i) & =(1+\beta) \ln \left(w_{t}\right)+\beta \ln \left(R_{t+1}\right)+(1+\beta) \ln \left(\frac{w_{t}(s, i)}{w_{t}}\right) \\
& +\beta \ln (\beta)+(1+\beta) \ln \left(1-\tau_{t}\right)+(1+\beta) \ln \left(\frac{\alpha+(1-\alpha) \tau_{t}}{\alpha(1+\beta)+(1-\alpha)\left(1-\kappa_{t+1}\right) \tau_{t+1}}(2) 8\right)
\end{aligned}
$$




\subsection{Comparative Statics: An Increase in the Migration Rate $\mu$}

In this subsection we derive the comparative statics of the model with respect to the migration rate $\mu_{t}$ and the fertility rate (population growth rate) $\gamma_{t}^{n}$ of the native population. From propositions 2 and 3 we know that these are completely determined by the demographic factors driving relative wages $\mathcal{W}_{h i}\left(\mu_{t} / \gamma_{t}^{n}\right), \mathcal{W}_{n a}\left(\mu_{t} / \gamma_{t}^{n}\right)$ as well as the growth in aggregate labor $\gamma_{t+1}^{L}\left(\mu_{t}, \gamma_{t}^{n}\right)$. The following lemma, proved in appendix A, summarizes the impact of migration and fertility rates on these exogenous demographic factors.

Lemma 1. Consider a change in the migration and/or native fertility rate $\left(\mu_{t}, \gamma_{t}^{n}\right)$

1. The relative wage factors $\mathcal{W}_{h i}\left(\mu_{t} / \gamma_{t}^{n}\right), \mathcal{W}_{n a}\left(\mu_{t} / \gamma_{t}^{n}\right)$ are strictly increasing in $\mu_{t} / \gamma_{t}^{n}$ and $\mathcal{W}_{l o}\left(\mu_{t} / \gamma_{t}^{n}\right)$ is strictly decreasing in $\mu_{t} / \gamma_{t}^{n}$.

2. Suppose the changes in $\mu_{t}, \gamma_{t}^{n}$ are permanent. Then the growth rate of aggregate labor $\gamma_{t+1}^{L}\left(\mu_{t}, \gamma_{t}^{n}\right)$ is strictly increasing in $\mu_{t}, \gamma_{t}^{n}$, and the share of migrants in labor $\frac{N_{t}(0, l o, \text { fo })}{L_{t}}$ and the resource cost of migration $\kappa_{t}$ are strictly increasing in $\mu_{t}$.

Equipped with this result and propositions 2 and 3 we now can state

Theorem 1. Consider an unexpected but permanent increase in the migration rate $\mu_{t}$.

1. First consider a small open economy:

(a) Welfare of all young native households is negatively impacted by an increase in the effective cost from migrants $\kappa_{t+1}$, positively impacted by an increase in the relative return on social security $\frac{\gamma_{t+1}^{L}}{R}$. The relative wage effect $\mathcal{W}_{h i}\left(\mu_{t} / \gamma_{t}^{n}\right)$ is unambiguously positive for high-skilled natives, but $\mathcal{W}_{l o}\left(\mu_{t} / \gamma_{t}^{n}\right) \cdot \mathcal{W}_{n a}\left(\mu_{t} / \gamma_{t}^{n}\right)$ is ambiguous for low-skilled natives.

(b) Therefore as long as migrants are not too costly $\left(\tilde{\kappa}_{t+1}\right.$ and thus $\kappa_{t+1}$ is sufficiently small), welfare of young high-skilled natives at the time of the migration boom, $U_{t}(h i, n a)$ increases due to the boom.

(c) The welfare consequences for young low-skilled natives $U_{t}(l o, n a)$ are ambiguous, but positive as long as their relative wages do not decline too much. This is true as long as long as $\sigma_{n f}$ is sufficiently small relative to $\sigma_{l h}$.

2. In general equilibrium the migration cost and the relative wage effects are identical to those in the small open economy and the impact on the relative return on social security $\frac{\gamma_{t+1}^{L}}{R}$ is absent. The wage level $w_{t}$ falls and the real return $R_{t+1}$ increases, and the overall general equilibrium effect $(1+\beta) \ln \left(w_{t}\right)+\beta \ln \left(R_{t+1}\right)$ is negative as long as 
the capital share $\alpha$ is sufficiently large or the increase in labor in $t$ and $t+1$ is of similar magnitude. ${ }^{8}$ In this case the welfare consequences from the migration boom shift down for all groups relative to the small open economy.

The proof follows directly from lemma 1 as well as propositions 2 and 3 . A similar theorem can be derived for a decline in the population growth rate of the native population. The main upshot of the simple model is that the welfare consequences of the 2015-106 immigration boom depends on four factors: i) the relative wage effects determined by the relative substitution pattern of skilled, unskilled native and skilled native labor, ii) the adjustment of the PAYGO pension system, iii) the costs to administer the migration system and iv) general equilibrium level effects on wages and interest rates. We now seek to quantify these effects in a more realistic large-scale overlapping generations economy.

\section{The Quantitative Model}

The quantitative model we employ is a large-scale overlapping generations model in the tradition of Auerbach and Kotlikoff (1987), but with time-varying, deterministic demographic structure. The key model ingredients are i) a detailed demographic model that accurately describes migrant flows into and out of the country, ii) a production technology that allows for flexible substitution patterns across workers with different skills and migratory backgrounds and leads to relative wages that depend on the relative labor supplies of the different population groups, iii) households with consumption-savings and labor supply decisions, and iv) a government that administers the migration system, a basic social insurance system, a pay-as-you-go social security scheme, and that collects taxes.

We now first describe the underlying demographic model that captures the flow of migrants and asylum seekers into Germany. We then turn to the description of the economic model, its production technology, endowments and preferences of households, as well as government policies. The recursive formulation of the household problem and the formal definition of equilibrium is relegated to the appendix. To provide an overview and set up notation, Table 1 summarizes the state variables used in the model.

\subsection{Demographics and Population Flows}

We distinguish between the native population and the foreign-born population. Foreigners are composed of those that entered the country as regular immigrants and those that came

\footnotetext{
${ }^{8}$ The appendix provides conditions on fundamentals for this to be true; they are easily verifiable in the quantitative model.
} 
Table 1: List of State Variables

\begin{tabular}{ccc}
\hline \hline State Var. & Values & Interpretation \\
\hline$j$ & $j \in\{0,1, \ldots, J\}$ & Model Age \\
$s$ & $s \in\{l o, m e, h i\}$ & Skill (education) \\
$i$ & $i \in\{n a, h o, r w, a s\}$ & Nationality \\
$g$ & $g \in\{f e, m a\}$ & Gender \\
$a$ & $a \geq 0$ & Assets \\
$e$ & $e \in\{e m, r e\}$ & Labor Market Status \\
\hline \hline
\end{tabular}

Notes: List of state variables of the economic model. lo: low, me: medium, hi: high eduction; na: natives, ho: high income OECD, HIOECD; rw: rest of the world, RW; as: asylum seekers, AS; fe: female, ma: male; em: employed, re retired.

as refugees and are asylum seekers. ${ }^{9}$ The basic difference between native households and foreigners is in their labor productivity, their access to government transfers as well as the fact that labor inputs supplied by natives and foreigners are imperfect substitutes in production.

We consider four nationalities denoted by $i \in\{n a, h o, r w, a s\}$, where nationality determines an individual's labor productivity and the extent to which individuals have access to the German social insurance system. Within the group of regular immigrants we distinguish between the population from high income OECD countries $(h o)$ and the rest of the world $(r w)$. Each population group is composed of three education groups, the low, medium, and high skilled, denoted by $s \in\{l o, m e, h i\}$. Within each group we consider females and males, $g \in\{f e, m a\}$. We assume that mortality and fertility rates are homogeneous across skill groups.

Households are born at age $j=0$ and live at most until age $J>0$. The number of people alive at time $t$, of age $j$, nationality $i$, and gender $g$ is denoted by $N_{t}(j, i, g)$. Households may die from $t, j$ to $t+1, j+1$ and we denote the according exogenous survival rates by $\psi_{t}(j, i, g)$. Further, we denote by $\mu_{t}(j, i, g)$ the net migration rate, i.e., the percentage net addition to the stock of $(j, i, g)$ type individuals from period $t$ to $t+1$, which accommodate leaving and assimilation flows: Each period the stock $N_{t}(j, a s, g) \psi_{t}(j, a s, g)$ of asylum seekers that survives to the next period faces the probability $\pi^{l}$ of having to leave the country. Those that stay face a probability $\pi^{a r}$ to assimilate to population group $r w$. Likewise, we assume that in each period a fraction $\pi^{r h}$ of the stock of population group rw assimilates to population group ho. Appendix B.1 describes how we accordingly construct the net

\footnotetext{
${ }^{9}$ We use the terms "refugees" and "asylum seekers" interchangeably. Empirically the latter group includes all successful asylum seekers as well as those waiting for a decision of their application, and finally those that have either been denied protection or lost their humanitarian residence title but remain in the country.
} 
migration rates $\mu_{t}(j, i, g)$. The dynamics of the size of each population group then obey

$$
N_{t+1}(j+1, i, g)=\left(\psi_{t}(j, i, g)+\mu_{t}(j, i, g)\right) N_{t}(j, i, g)
$$

where $\psi_{t}(J, i, g)=\mu_{t}(J, i, g)=0$.

Denote by $\chi_{t}(j, i)$ the time $t$, age $j$, group $i$ specific fertility rate and by $\phi$ the exogenous fraction of baby girls, assumed to be constant over time and across population groups. We further assume that all newborns of group $i$ are natives. We denote by $j_{f}$ the first $f$ ertile age of a woman and by $j_{c}$ the age of completed fertility. The number of native newborns of gender $g$ in period $t+1$ is then given by

$$
\begin{aligned}
N_{t+1}(0, n a, f e) & =\phi \sum_{i \in\{a a, h o, r w, a s\}} \sum_{j=j_{f}}^{j_{c}} \chi_{t}(j, i) N_{t}(j, i, f e) \\
N_{t+1}(0, n a, m a) & =(1-\phi) \sum_{i \in\{n a, h o, r w, a s\}} \sum_{j=j_{f}}^{j_{c}} \chi_{t}(j, i) N_{t}(j, i, f e) .
\end{aligned}
$$

Since all babies born in Germany are treated as natives, the foreign age 0 population groups are those that migrated from $t$ to $t+1$ to Germany as babies, thus for $i \in\{h o, r w, a s\}$

$$
N_{t+1}(0, i, g)=M_{t+1}(0, i, g) .
$$

\subsection{Technology}

Output $Y_{t}$ is produced with a neoclassical production function that displays constant returns to scale in capital $K_{t}$, a labor aggregate $L_{t}$ and a technology level $A_{t}$, which grows at constant rate $\lambda$. Firms operate in perfectly competitive output and factor markets, and thus earn zero profits in equilibrium. Given these assumptions, without loss of generality we consider the problem of a representative firm. We assume a Constant Elasticity of Substitution (CES) aggregate production function of the form

$$
Y_{t}=\left(\alpha K_{t}^{1-\frac{1}{\vartheta}}+(1-\alpha)\left(A_{t} L_{t}\right)^{1-\frac{1}{\vartheta}}\right)^{\frac{1}{1-\frac{1}{\vartheta}}},
$$

where $\vartheta$ is the substitution elasticity between capital and the labor aggregate. 
Aggregate labor in turn is a CES aggregate of labor supplied by the different skill groups $s \in\{l e, m e, h i\}, L_{t}(s)$, with substitution elasticity $\sigma_{l m h}$ :

$$
L_{t}=\left(\sum_{s \in\{l o, m e, h i\}} L_{t}(s)^{1-\frac{1}{\sigma_{l m h}}}\right)^{\frac{1}{1-\frac{1}{\sigma l m h}}} .
$$

Next, labor of skill group $s$ is the aggregate of different age (experience) groups $\bar{j}$ which we model as perfect substitutes,

$$
L_{t}(s)=\sum_{\bar{j}=1}^{n_{\bar{j}}} L_{t}(\bar{j}, s)
$$

where $n_{\bar{j}}$ denotes the number of experience groups. These education and experience group specific labor inputs are the CES aggregate of natives and foreigners with substitution elasticity $\sigma_{n f}$ :

$$
L_{t}(\bar{j}, s)=\left(L_{t}(\bar{j}, s, n a)^{1-\frac{1}{\sigma_{n f}}}+\tilde{L}_{t}(\bar{j}, s, f o)^{1-\frac{1}{\sigma_{n f}}}\right)^{\frac{1}{1-\frac{1}{\sigma_{n f}}}}
$$

where $\tilde{L}_{t}(\bar{j}, s, f o)$ is a CES aggregate of foreigners given by

$$
\tilde{L}_{t}(\bar{j}, s, f o)=\left(L_{t}(\bar{j}, s, h o)^{1-\frac{1}{\sigma_{h r}}}+\left(\sum_{i \in\{r w, a s\}} L_{t}(\bar{j}, s, i)\right)^{1-\frac{1}{\sigma_{h r}}}\right)^{\frac{1}{1-\frac{1}{\sigma_{h r}}}} .
$$

We thus assume that conditional on education and experience, population groups $r w$ and as are perfect substitutes in production, ${ }^{10}$ whereas the composite of this group and foreigners from group ho are imperfect substitutes with substitution elasticity $\sigma_{h r}$. Those skill, experience group, and nationality specific labor inputs are aggregates of productivity-weighted age-specific hours worked by men and women. We assume perfect substitution across age and gender, so that we have

$$
L_{t}(\bar{j}, s, i)=\sum_{g \in\{f e, m a\}} \sum_{j=j_{l}(\bar{j}, s)}^{j_{h}(\bar{j}, s)} \epsilon(j, s, i, g) L_{t}(j, s, i, g),
$$

\footnotetext{
${ }^{10}$ This is due to data limitations since we do not separately observe asylum seekers for all sample years used in estimation of the production function, which inhibits the estimation of a substitution elasticity parameter.
} 
where $\left[j_{l}(\bar{j}, s), \ldots, j_{h}(\bar{j}, s)\right]$ is the education-specific age bracket of experience group $\bar{j}, L_{t}(j, s, i, g)$ are hours worked, and $\epsilon(j, s, i, g)$ is labor productivity of age, skill, nationality and gender group $j, s, i, g$. We further assume a constant gender specific shifter of productivity profiles, which gives productivity as $\epsilon(j, s, i, g)=\epsilon(j, s, i) \epsilon(g)$. Finally, capital is assumed to depreciate at constant rate $\delta$. The first-order conditions of the firm problem are provided in Appendix B.

\subsection{Households}

\subsubsection{Timing of Work and Retirement}

Agents (females and males) work from age $j_{a}$ until at most the mandatory retirement age $j_{r}$. At age $j_{a}$ the idiosyncratic elements of the wage process $\epsilon(j, s, i, g)$ realize. Skill $s$ is exogenously given at economic birth and we do not consider any inter-generational spillovers of skills. There are three skill levels, low, medium and high, $s \in\{l o, m e, h i\}$. While agents know their skill levels already at age $j_{a}$, for an initial working period $j \in\left\{j_{a}, \ldots, j_{s}\right\}$ agents of skill $s$ lose fraction $\varrho(s) \in(0,1)$ of labor productivity, standing in for time spend on formal education, whereby the "education period" increases in the skill level, $j_{h i}>j_{m e}>j_{l o}=j_{a}-1$. Labor supply of groups $i \in\{n a, h o, r w\}$ can be chosen from the discrete set $\left\{l_{1}, \ldots, l_{n}\right\}$. Asylum seekers instead are restricted to supply an exogenous amount of hours. In the first year of arrival asylum seekers only work amount $\underline{l}^{a}$, and after the first year those that have not assimilated and thus stayed in nationality group as work amount $\bar{l}^{a}>\underline{l}^{a}$. All agents retire exogenously at age $j_{r}<J$, and we denote the employment status by $e \in\{\mathrm{em}, \mathrm{re}\}$, where $\mathrm{em}$ is employment, re is retirement.

\subsubsection{Endowments}

Agents are endowed with one unit of productive time. An agent of skill $s$, age $j$, nationality $i$, gender $g$ earns an hourly gross wage of

$$
w_{t}(j, s, i) \epsilon(j, s, i) \epsilon(g)
$$

where $w_{t}(j, s, i)$ is the age $j$, education $s$ and nationality $i$ specific aggregate wage component, determined in equilibrium, $\epsilon(j, s, i)$ is age-, skill-, nationality-specific productivity, and $\epsilon(g)$ is the gender-specific productivity component, see Section 5.2. Whereas agents know their education at age $j_{a}$, agents at age $j_{a} \leq j \leq j_{s}$ are still in formal education and thus experience a reduction of their productivity by factor $\varrho(s)$. 
In retirement, agents receive a pension income, which depends on all fixed observable characteristics that measure productivity, $(s, i, g)$, to proxy the non-redistributive elements of the German pension system. This component of pension income is denoted by $p(s, i, g)$. Pension benefits are further indexed by the benefit level $\rho_{t}$, which adjusts to clear the aggregate pension budget in each period, so that pension income is

$$
b_{t}^{p}(s, i, g)=\rho_{t} \cdot p(s, i, g) \text {. }
$$

Agents start their economic life with zero assets. From then on they have access to a risk-free savings technology with gross interest rate $r_{t}$. In case of death assets are confiscated and redistributed as accidental bequests, lump-sum across the population alive in period $t, t r_{t}$. For asylum seekers, we distinguish between the first period in which they are asylum seekers and all other periods in which they are accepted or tolerated (we do not distinguish between those). ${ }^{11}$ In that first period, under the restricted access to labor market with exogenous hours worked of $\underline{l}^{a}$, asylum seekers receive transfer payments $b_{t}^{a}(n)$, which are adjusted to household size by the number of children $n$. At the end of the first period, conditional on surviving to the next period they face the probability $\pi^{l}$ with which they have to leave the country and, conditional on staying, the probability $\pi^{a r}$ with which they assimilate to the foreign population group $r w$. The remaining fraction $\left(1-\pi^{l}\right)\left(1-\pi^{a r}\right)$ stays in state as as accepted or tolerated asylees. From now on, asylees who remain in state as have full access to the tax and transfer insurance scheme but their labor supply is fixed at $\bar{l}^{a}$, which is lower than for population group $r w{ }^{12}$ At the end of each period they continue to face the leaving and assimilation shocks conditional on surviving with respective probability $\pi^{l}$ and conditional probability $\pi^{a r}$. Asylees take into account the leaving probability in their decision problem and we assume that the continuation value when leaving is the lifetime utility from consuming the annuitized value of their total wealth in each period, assuming that they work full time each period, with all details provided in Appendix B. Similar to asylum seekers, foreigners from group $r w$ face a constant, state-independent probability of assimilation to population group ho, denoted by $\pi^{r h}$.

Finally, all agents pay contributions to a PAYG financed social security and health insurance, non-linear labor income taxes, consumption taxes and capital income taxes all of which we specify in Section 5.4.

\footnotetext{
${ }^{11}$ According to factual legislation, some are non-accepted asylees but are tolerated to stay. Of others the status may still be pending after one year. Economically, there is little difference across these different types.

${ }^{12}$ The focus of our analysis lies on the fiscal and general equilibrium consequences of refugee immigration, which is why it is unproblematic not to model the refugee decisions in the same way as for the other groups of the population.
} 


\subsubsection{Preferences}

Households derive per period utility from the consumption of a market good $c$ and leisure. The household period utility function for gender $g$ and skill $s$ at age $j$ is thus

$$
u\left(\frac{c}{1+\zeta n}, 1-l\right)
$$

where $\zeta$ is a child equivalence parameter and $n$ denotes the number of children of the household. The recursive formulation of the life-cycle household model is contained in Appendix B.5.

\subsection{Government}

There are three separate government budgets, one for the pension system, one for the health insurance system and one for the general tax and transfer system.

Pension System. Labor income is taxed at the linear rate $\tau_{t}^{p}$ to finance pension income. We assume that all contributions to the pension system are paid by workers and are taxexempt. We also assume a balanced budget in the pension system, thus in each period the sum of contributions is equal to all layouts.

Health Insurance System. In addition, earnings (labor income and pension income) are taxed at rate $\tau_{t}^{h}$ to finance average age, gender, and time-specific health expenditures of households $b_{t}^{h}(j, g)$. These transfers are used to cover health expenditures that perfectly restore the health stock. Thus, at the household level expenditures on health and transfer payments received net to zero, and the average transfer payments $b_{t}^{h}(j)$ only show up explicitly in the budget of the health care system, which is balanced each period.

General Tax and Transfer System. The government also collects linear taxes on consumption and on capital at rates $\left(\tau_{t}^{c}, \tau_{t}^{k}\right)$. Labor income (net of pension contributions) and pension income is taxed through a non-linear labor income tax code $T_{t}\left(y_{t}\right)$, where $y_{t}$ is taxable income of the household, i.e., labor income net of pension contributions during the working period and pensions in retirement. Government revenues net of transfers implied by tax code $T_{t}\left(y_{t}\right)$ are used to finance an exogenous stream of government expenditures $G_{t}$, transfers to asylum seekers, $b_{t}^{a}$, and transfers to leavers, $b_{t}^{l}$, all of which we aggregate in total transfers $Z_{t}$, as well as administrative expenses per asylum seeker $g_{t}^{a}$, aggregated as $E_{t}$. 


\section{Calibration}

Most parameters are calibrated externally to aggregate data or are based on our direct empirical estimates. We calibrate endogenously the discount factor $\beta$, gender specific leisure weights in the utility function and the level parameter of the income tax function. These parameters are determined jointly so that the benchmark model in a steady state of 2010 matches moments for the period 1980-2010. ${ }^{13}$ A summary of the calibration is contained in Table 5, provided in Appendix C.

\subsection{Time}

We assume that the economy is in an initial steady state in 1960 (model period $t=0$ ), the first year for which we have comprehensive population data from the Human Mortality Database (HMD). On the basis of the HMD mortality rates we compute in 1960 a steady state population distribution with a constant population, taking as given the size of the age-0 population in 1960. From 1961 on we take actual population data, and thus assume that, by surprise, the economy jumps to the actual demographic dynamics from beginning of year 1961. The period until (including) year 2012 is a phase-in period, through which we make sure that the demographic distribution in the model is consistent with the actual demographic dynamics in the data, and that the dynamics of macroeconomic aggregates and their distribution in the period of interest starting in 2013 is not affected by our initialization in 1960-61.

From 2013 on we consider three demographic scenarios across which we vary the size of predominantly low-skill immigration to Germany. As our baseline scenario we assume time-varying and age-specific mortality- and fertility rates and take time-varying migration numbers as observed in the data, but ignore the migration inflow of population groups $r w$ and as from 2013-2018. The high refugee migration scenario takes the immigration by the population of asylum seekers, and the high migration scenario additionally includes the higher migration numbers from population group $r w$ in those years. In all scenarios we model the additional inflow of immigrants as a zero probability event, and assume a trend reversal to long-run average migration from 2018 to 2022, assuming zero emigration of natives.

\footnotetext{
${ }^{13}$ We compute a year 2010 steady state for calibration purposes only. Otherwise year 2010 is a year in our transition of the phase-in period.
} 


\subsection{Population}

The details underlying the population dynamics in all three demographic model variants are described in Appendix C.1. Figure 2 shows the spike in the inflow of asylum seekers from 2013 to 2017 , and the fact that most of these migrants are 20 to 40 years old. ${ }^{14}$

Figure 2: Net Migration, Population Group as

(a) Aggregate Inflow

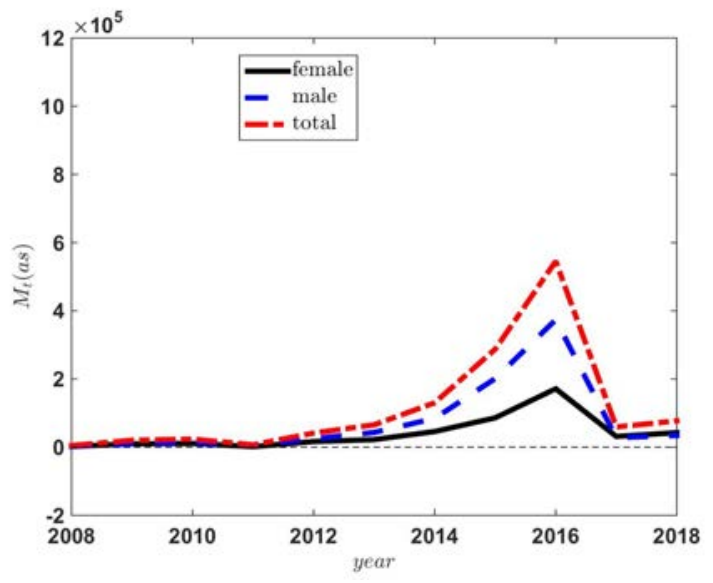

(b) Age Distribution

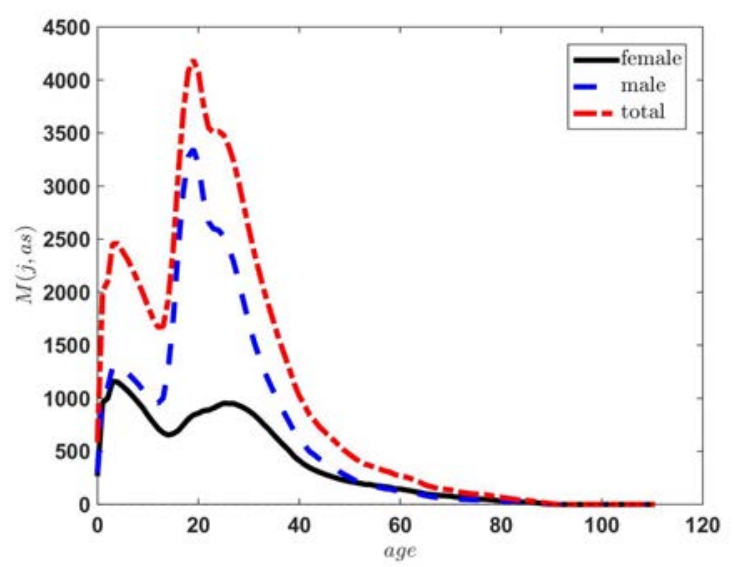

Notes: Migration inflow of asylees. Panel (a): aggregate net immigration, Panel (b): average age distribution of net immigration during 2008-2018. Source: Own calculations based on Central Foreign Population Registry (Ausländerzentralstatistik, AZR).

Figure 3 shows the corresponding age distribution in the native population and in the population of group as; the respective distributions for the other population groups, ho and $r w$, are shown in Appendix C, Figure 15. Overall, the foreign population stock is much younger than the native population.

To predict future mortality rates we extract data from the Human Mortality Database, estimate a Lee-Carter model (Lee and Carter 1992) and hold predicted mortality rates constant from year 2100 onwards. Finally, we combine data from the Federal Statistical Office on age-specific fertility rates with the population stock data and the number of births to determine age and time specific fertility distributions. We assume that those are identical for all groups and adjust them such that from 2100 onwards the number of newborns is constant. The model reaches a stationary population distribution by about $2200 .{ }^{15}$

\footnotetext{
${ }^{14}$ Numbers for the other population groups are contained in Figures 13 and 14 of the Appendix.

${ }^{15}$ During the phase-in period from 1960 to 2012 we have the exact data on the separate population stocks only from 2008 onwards. Leading towards 2008 we forward shoot on the population dynamics using data on the annual flow of migration and distribute those across the four groups such that we minimize the distance between the model implied population stocks in the four groups in 2008 and the respective actual population stock.
} 
Figure 3: Age Distribution of Population Stock

(a) Group na

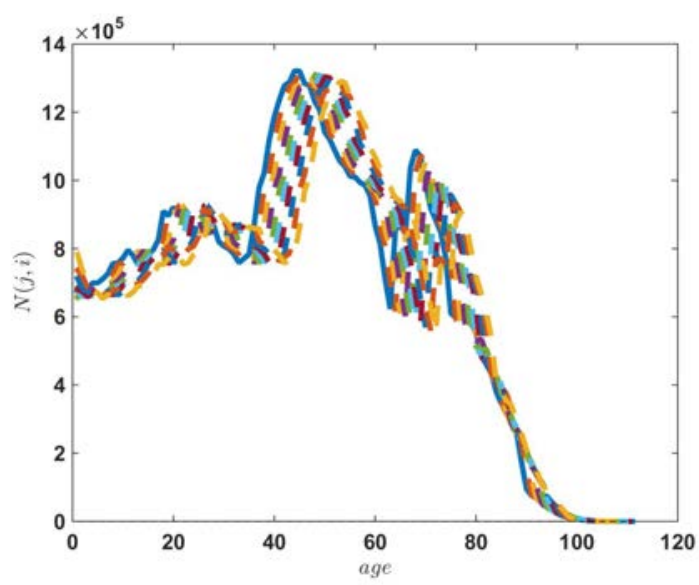

(b) Group as

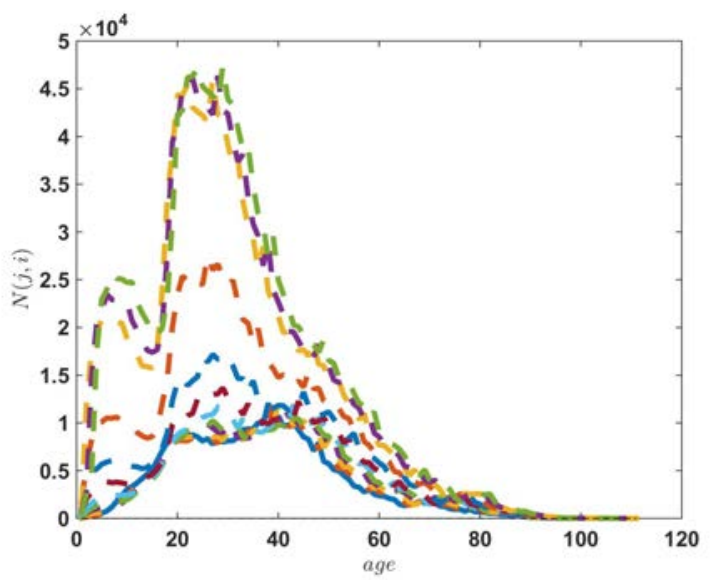

Notes: Age distribution in the population during 2008-2018 among natives and foreigners of the population group asylees. Source: Own calculations based on Central Foreign Population Registry (Ausländerzentralstatistik, AZR) and German Federal Statistical Office (Statistisches Bundesamt).

\subsection{Age and Experience}

Households start their economic life at age $j_{a}=17$, thus education of the low skilled is completed at age $j_{l o}=16$. We set the age at which medium-skilled and high-skilled complete education to $j_{m e}=20$ and $j_{h i}=24$, respectively. Consistent with the data on fertility rates the first fertile year is $j_{f}=15$, and fertility is completed by the age of $j_{c}=50$. The fixed retirement age is $j_{r}=66$, and the maximum age is $J=100$.

We consider three experience groups of workers, $n_{\bar{j}}=3$, for years of experience $1-9$, $10-19$, and $\geq 20$ years, assume that during education workers do not accumulate experience and, since labor market experience is not a state variable in our model, experience increases linearly with age after completion of education.

\subsection{Firms}

We follow the literature (cf., e.g., Borjas 2003) to estimate the elasticities of substitution in production, summarized in Table $2 .{ }^{16}$ These estimates point to a relatively low degree of substitutability across education groups, consistent with estimates from other studies for Germany. ${ }^{17}$ The obtained elasticity of substitution between natives and immigrants lies

\footnotetext{
${ }^{16}$ The point estimates of the inverse (and negative) of the elasticities with clustered standard errors in parentheses are $\sigma_{l m h}=-0.33(0.12), \sigma_{n f}=-0.08(0.01)$, and $\sigma_{h r}=-0.04(0.01)$.

${ }^{17}$ The point estimate in D'Amuri et al. (2010) is 2.0, while Brücker and Jahn (2011) estimate an elasticity of about 6.5. For the United States, estimates are typically slightly lower: Borjas (2003) estimates an
} 
in between similar estimates for Germany found in the literature. ${ }^{18}$ Finally, the degree of substitutability within the group of foreigners is highest.

Table 2: Substitution Elasticities in Production

\begin{tabular}{cccc}
\hline \hline & Education $\left(\sigma_{l m h}\right)$ & Foreigners/Natives $\left(\sigma_{n f}\right)$ & Within Foreigners $\left(\sigma_{h r}\right)$ \\
\hline$\sigma$ & 3.05 & 13.22 & 22.61 \\
\hline \hline
\end{tabular}

Notes: Substitution elasticities in aggregate production. $\sigma_{l m h}$ : s.e. between lo, medium, high skilled; $\sigma_{n f}$ : s.e. between natives and foreigners; $\sigma_{h r}$ : s.e. between HIOECD and RW. Source: Own calculations based on SOEP.

In terms of the remaining parameters of the production function we set $\alpha=0.33$ and set $\delta=0.05$. The rate of exogenous technological progress is set to $\lambda=0.015$. Throughout, we detrend the economy by the technology level and thus can think of model wages as detrended. We normalize the initial 2010 technology level $A_{0}$ such that the detrended aggregate wage rate is equal to one in that year. We also normalize the age $j$, education $s$, group $i$, gender $g$-specific productivity profiles $\epsilon(j, s, i, g)$ so that in the 2010 steady state of the model we match the wage premia across groups that are implied by the age wage profiles $w(j, s, i) \epsilon(j, s, i, g)$.

\subsection{Households}

\subsubsection{Preferences}

The per-period utility function features logarithmic utility from consumption and linearadditive logarithmic utility from leisure according to

$$
\ln (c)+\phi(g) \ln (1-l(g))
$$

with gender specific leisure share parameters $\phi(g)$. We discretize the labor supply choice into three possible options for low participation, part-time and full-time work. For a week with 112 hours of available time (corresponding to seven days with 16 hours each), a full time job with 40 hours corresponds to $36 \%$ of time endowment. We accordingly set $l(g) \in$ $\{0.036,0.18,0.36\}$. Given this choice set, we calibrate $\phi(g)$ to match average hours worked in the period 2000-2009 of 25-65 year olds in the SOEP by gender $g \in\{f e, m a\}$. A worker

elasticity of about 1.3, and Katz and Murphy (1992) of about 1.4. This is not surprising since we use three education groups for Germany, whereas the US estimates are based on two education categories.

${ }^{18}$ Felbermayr et al. (2010) find a substitution elasticity around 7, D'Amuri et al. (2010) of about 22, and Brücker and Jahn (2011) about 15-20, respectively. For comparison, Ottaviano and Peri (2008) also find a slightly higher estimate of around 20 for the US. 
is counted as participating if she works at least 520 hours in a given year. We compute gender specific participation rates and annual hours worked conditional on participation in the SOEP and translate the resulting unconditional annual hours into fractions of maximum annual work hours of 2080 (52 weeks with 40 hours per year). This gives fractions of full time hours of $47.5 \%$ for females and of $81.5 \%$ for males, our calibration targets for the nationality specific leisure shares $\phi(g)$. The resulting parameter values are $\phi(f e)=2.846$ and $\phi(m a)=1.227$.

Households discount future utility at rate $\beta$. We calibrate it to match a rate of return on capital of $4 \%$, which, given a depreciation rate of $5 \%$, is consistent with a capital to output ratio of 3.6. This gives $\beta=0.983$.

\subsubsection{Skill Distribution}

On the basis of the population of the 26-60 year olds in the SOEP we estimate the skill distribution by gender and nationality group, summarized in Table 3. The high-skilled are graduates from university and college, medium skill refers to the various vocational degrees of the German education system, and low skilled are those with only general elementary schooling or without any formal education. Observe that the biggest difference between the native population and immigrants is a relatively low fraction of the population being low skilled. Among group as about 50\% have no formal education, and are thus in the low skill group.

Table 3: Skill Distribution in Population

\begin{tabular}{lcccccc}
\hline \hline & \multicolumn{3}{c}{ Females } & \multicolumn{3}{c}{ Males } \\
\hline Educ $s$ / Region $i$ & low & medium & high & low & medium & high \\
\hline Natives & 0.0954 & 0.7457 & 0.1589 & 0.0525 & 0.7151 & 0.2324 \\
Foreigners $h o$ & 0.2458 & 0.5829 & 0.1713 & 0.2362 & 0.5839 & 0.1799 \\
Foreigners $r w$ & 0.2588 & 0.5388 & 0.2023 & 0.1921 & 0.6803 & 0.1276 \\
Foreigners as & 0.5270 & 0.2580 & 0.2150 & 0.5019 & 0.2381 & 0.2600 \\
\hline \hline
\end{tabular}

Notes: Shares with education $s \in\{l o, m e, h i\}$ among population groups $i \in\{n a, h o, r w, a s\}$ by gender. Source: Own calculations based on SOEP.

\subsubsection{Wage Process}

Recall from equation (38) that hourly wages are

$$
w_{t}(j, s, i) \epsilon(j, s, i) \epsilon(g) \text {. }
$$


We normalize male efficiency units $\epsilon(m a)=1$ and estimate $\epsilon(f e)=0.8074$ for average female efficiency. The proportional productivity loss while still obtaining education is set to $\varrho(s)=0.5$, for $s \in\{m e, h i\}$. As discussed in Section 5.2, asylum seekers and foreigners from the "rest of the world" are perfect substitutes, an assumption we make for reasons of data limitations. We estimate the productivity of asylum seekers relative to "rest of the world" foreigners, which we also translate into the calibrated assimilation probability $\pi^{a r}$ in Section 6.5.4. For the remaining groups $i \in\{n a, h o, r w\}$, the productivity profiles $\epsilon(j, s, i)$, respectively age wage profiles $w(s, i) \epsilon(j, s, i)$, are estimated from individual level data by Mincer regressions of log wages on population group and education specific second-order polynomials in age and a set of fixed effects. ${ }^{19}$ Figure 16 in Appendix C displays the age wage profiles for natives in Panel (a) and foreigners from group "rest of the world" in Panel (b) for the three education categories $s \in\{l o, m e, h i\} .{ }^{20}$

\subsubsection{Immigrants' Unemployment, Assimilation and Leave Probabilities}

We base the calibration of the probability $\pi^{a r}$ of nationality group as assimilating to nationality group $r w$, and of the probability $\pi^{r h}$ of group $r w$ assimilating to ho on estimates of relative (residual) wages as a function of years since immigration. We take a sample from SOEP of males who have completed their education and who work at least 520 hours. We first purge log wages from a set of explanatory variables, ${ }^{21}$ and then fit a linear regression of residual wages on years since immigration separately for the three immigrant groups. The fitted regressions imply a wage gap upon immigration of as to $r w$ immigrants of $23.76 \%$, which gives $\epsilon(j, s, a s)=0.7624 \cdot \epsilon(j, s, r w)$. Fitting a linear function to the relative productivity of as to rw over years since immigration implies that this gap would hypothetically be closed half-way after about 87 years. This translates into an annual assimilation probability of $\pi^{a r}=0.8 \%$. By the same logic, we estimate a half-time of the relative productivity of $r w$ to ho of about 11 years, which gives an annual assimilation probability of $\pi^{r h}=6.0 \%$.

To compute the emigration probability $\pi^{l}$, we take data on the flow of leavers from the Federal Office for Migration and Refugees (Bundesamt für Migration und Flüchtlinge, BAMF). Since we do not distinguish in our analysis between involuntary and voluntary leaves, we base our calculation on the total number of leaves. We relate those to the stock

\footnotetext{
${ }^{19} \log$ wages are adjusted by the gender-specific productivity shifter from the production function estimation, and the Mincer regressions are specified to be exactly consistent with the first order conditions of the firm problem. Given persistent wage differences between East and West Germany, see Heise and Porzio (2019), we also adjust wages for an East German wage penalty.

${ }^{20}$ Population group "high income OECD countries", ho, is in between.

${ }^{21}$ We control for year fixed effects, education fixed effects, quadratic polynomials over time and age for each education group, age fixed effects, a West Germany fixed effect, household size, marital status, and marriage and divorce effects.
} 
of asylum seekers in a given year for the period 2008-2018. On average, about 6\% leave, and there is little variation over the 10 years of data. We thus take $\pi^{l}=0.06$. All probabilities, i.e., $\pi^{a r}, \pi^{r h}, \pi^{l}$, apply only up to age $j_{r}-1=65$.

In case of leaving, asylum seekers are also assumed to have lower labor productivity in the destination country, which is reflected in parameter $\eta$. Since most asylum seekers came from Syria, we proxy productivity differences by estimating aggregate production functions for Germany and Syria and by comparing the resulting Solow residual. This gives $\eta=0.45$. The calculation of the annuity stream of leavers is outlined in Appendix B.

\subsubsection{Participation Rates of Asylum Seekers}

For asylees, we estimate labor market participation as a function of years since immigration for the refugee cohort under study. We proceed in two steps. First, we estimate a Linear Probability Model of participation using micro data on immigrants including the recent refugee wave. Second, we use this model to predict the participation rate in the incoming refugee cohort in our simulation, using the distribution of refugees over education, age, and sex at the peak of the refugee immigration in 2015.

Our estimation is based on data from the IAB-SOEP migration, and the IAB-BAMFSOEP refugee samples. From those samples we use data for sample years 2014-2017, and we consider immigrants that immigrated in 2010 or later. We then regress participation ${ }^{22}$ on years since immigration and a refugee fixed effect (also controlling for age groups and education groups). Using the estimated model gives an initial participation among the 2565 year old refugees of $16.6 \%$. After seven years, the predicted participation is at $43.2 \%$. We translate these values of participation into the low and high hours states $\underline{l}^{a}$ and $\bar{l}^{a}$, respectively, by assuming that conditional on participation asylum seekers work the same number of hours as members of nationality group $r w$. Based on the average unconditional hours in group rw our estimates of participation rates imply that in the first year refugees work $10.9 \%$, and in all other years they work $36.9 \%$ of full time, our values of the respective model parameters $\underline{l}^{a}$ and $\bar{l}^{a}$ (expressed as fractions of full time work). Note that by our assumption of stochastic assimilation, the model counterpart to the hours worked of refugees after seven years is a weighted average of immigrant-group-specific hours worked. ${ }^{23}$

\footnotetext{
${ }^{22}$ For the purpose of the estimation, participation is a dummy variable that takes on value 1 if the indicated primary activity at the time of the survey is working.

${ }^{23}$ Consider a cohort of asylees. Given that each year a fraction $\pi^{a r}=0.008$ of as assimilate to $r w$, and in turn a fraction $\pi^{r h}=0.06$ of $r w$ assimilate to $h o$, in expectation, after seven years $94.6 \%$ of the initial asylees will still be in group as, $4.5 \%$ will be in group $r w$, and $0.9 \%$ will be in group $h o$.
} 


\subsubsection{Taste Shocks}

Throughout we adopt taste shocks to smooth out the discrete choice decision problems. Specifically, we assume that at the beginning of a period, if a household chooses between discrete labor supply options $l \in\left\{l_{1}, \ldots, l_{n}\right\}$ then she solves the problem

$$
V_{t}(S, \varepsilon)=\max \left\{J_{t}\left(S, l_{1}\right)+\varsigma \varepsilon\left(l_{1}\right), \ldots, J_{t}\left(S, l_{n}\right)+\varsigma \varepsilon\left(l_{n}\right)\right\}
$$

where, given state variables $S$, the function $V_{t}(S, \epsilon)$ is the upper envelope of the labor choicespecific value functions $J_{t}\left(S, l_{i}\right)$, and $\varepsilon\left(l_{i}\right)$ is the realization of a taste shock obeying an extreme value type I (Gumbel) distribution with scale parameter $\varsigma$, cf., e.g., Iskhakov et al. (2017). Given this distributional assumption the expected value $\mathbb{E}_{\varepsilon}\left[V_{t}(S, \varepsilon)\right]$ and the corresponding choice probabilities have closed form expressions (McFadden 1973), which is convenient for the computation of the model. We set the smoothing parameter to $\varsigma=0.1$ for all discrete choice problems.

\subsection{Government}

\subsubsection{Transfer Payments to Asylees and Administrative Expenses}

Transfer Payments $b^{a}(\cdot)$. Our data on transfer payments to asylum seekers is based on the Asylum-Seekers' Benefits Act, which was introduced in 1993 to determine the entitlements for asylum seekers. ${ }^{24}$ The act applies to asylum seekers who filed an application for protection, those obliged to leave Germany as result of rejection of application and those with a temporary suspension of removal. ${ }^{25}$ We base our calibration on the most recent data for years 2011 to 2019. Based on this data the transfer schedule $b^{a}(n)$, taking the average over years in constant 2010 prices, a weighted average of schedules of couples and singles, assuming that transfers increase linearly in the number of children, is $b^{a}(n)=3809+2844 \cdot n$. We normalize benefits by average earnings in the model and the data and assume constant growth of transfer payments at the rate of technological progress $\lambda$.

\footnotetext{
${ }^{24}$ Consequently, data on transfer payments to asylum seekers is not available before 1994 .

${ }^{25}$ The benefits include food, housing, heating, healthcare, personal hygiene, assistance in sickness, pregnancy and birth as well as and household durables and consumables. In October, 2015 the law was revised and the level of social benefits were raised furthermore, it substituted 'in cash' benefits for 'in kind' benefits for asylum seekers staying in initial reception centers. Those moved to other decentralized locations receive benefits in cash or kind depending upon the decisions of local authorities which are regulated by Federal states. The 2015 reforms also state that the asylum seekers are entitled to standard social benefits and full healthcare after receiving social benefits under Asylum Seekers' Benefits Act for 15 months.
} 
Administrative Expenses $g^{a}$. In addition to the direct transfer payments to asylum seekers, estimated administrative and labor costs per asylum seeker are sizeable. Czerny (2019) estimates administrative costs in 2010 prices of 2908 Euro of annual labor costs and 4968 Euro of annual administrative costs per asylum seeker in prices of 2010. On this basis we set $g^{a}=7876$, which again is appropriately normalized and grows at constant rate $\lambda$.

Emigration Transfers $b^{l}$ The Reintegration and Emigration Programme for AsylumSeekers in Germany (REAG/GARP) provides financial support to asylum seekers and recognized refugees who volunteer to return to their country of origin or a third country of reception. ${ }^{26}$ The program provides assistance with travel cost, financial travel assistance, medical costs, one-time financial start-up assistance. ${ }^{27}$ The cost of assisted return per refugee grew from 686 Euro in 2003 to 1288 Euro per adult in 2008. The rise in cost is mainly due to a decrease in the number of returnees from 11835 in 2003 to 2799 in 2008 (BAMF).

In our model we do not distinguish between voluntary and involuntary leaves. ${ }^{28}$ We thus first compute total expenses in the years 2003 to 2008 and then divide them by the total number of leavers in these years. The resulting average for 2005-2008 in 2010 prices of is about 166 Euro per person. We thus set $b^{l}=166$, normalized and growing at rate $\lambda$, and do not distinguish between administrative costs and direct transfers to the refugees.

\subsubsection{Taxes and Government Expenditures}

We approximate the German labor income tax code by a parametric tax function, see Benabou (2002):

$$
T_{t}\left(y_{t}\right)=\left(\frac{y_{t}}{A_{t}}-\omega_{0}\left(\frac{y_{t}}{A_{t}}\right)^{1-\omega_{1}}\right) A_{t}
$$

where the parameter $\omega_{0}$ controls the level of taxes and $\omega_{1}$ determines the progressivity of the tax code. The trend adjustment by the technology level $A_{t}$ insures that taxes grow at the level of technology. Based on the estimates of Holter et al. (2019) we set $\omega_{1}=0.2035$, and adjust the tax level parameter $\omega_{0}$ to match an average ratio of government spending to GDP, $\frac{G_{t}}{Y_{t}}$, in the 2010 steady state of $19.12 \%$, implying $\omega_{0}=0.842$. Along the transition in the baseline

\footnotetext{
${ }^{26}$ There are also other programs that regionally operate in different states. REAG/GARP is the leading program recognized for assisted return of refugees and asylum seekers in Germany. Among other criteria the provision of these benefits depends on the nationality of the asylum seekers.

${ }^{27}$ Data on the number of departures under assisted return programs and related costs are not public. However, some figures can be extracted from the BAMF publications.

${ }^{28}$ The share of voluntary leavers to which these transfer payments apply fluctuates between about $10 \%$ and $40 \%$ with an average of $33 \%$ for $2000-2018$.
} 
demographic scenario we feed into the model the observed data on $\frac{G_{t}}{Y_{t}}$ until 2012 and hold it constant thereafter. The consumption tax rate is set to the current level of $\tau^{c}=19 \%$ in the steady state and adjusted along the transition to clear the government budget. Capital income taxes are held constant at $\tau_{t}^{k}=25 \%$, in line with current legislation. In the two alternative migration scenarios, our benchmark results are based on the assumption that spending $G_{t}$ is held constant at the path implied by the baseline demographic model. Of course, the migration-induced government outlays on refugees $E_{t}$ adjust to the elevated immigration flows. In a model with purely wasteful government spending such as ours it is a reasonable baseline to hold $G_{t}$ constant because a migration-induced increase of $G_{t}$ would only lead to tax increases, but would not yield utility benefits from increased public goods provision, neither for migrants nor the native population.

\subsubsection{Pension System}

To approximate the non-redistributive features of the German public pension system in our model without an explicit notion of a pension entitlement, we link the population-groupspecific component of individual pension income to group-specific wages and to age productivity as $p(s, i, j)=w_{t}(\bar{j}=3, s, i) \cdot \epsilon\left(j_{a}, s, i, g\right)$. We base the calibration of the contribution rate of the pension system on data up to 2012 and adjust the pension benefit level $\rho_{t}$ to clear the budget of the pension system. Thereafter, we apply the German pension adjustment formula so that after 2012 the pension benefit level $\rho_{t}$ evolves according to

$$
\rho_{t}=\rho_{t-1} \cdot \frac{1-\iota_{t}-\tau_{t}^{p}}{1-\iota_{t-1}-\tau_{t-1}^{p}} \cdot\left(\frac{R Q_{t}}{R Q_{t-1}}\right)^{-\alpha^{p}}
$$

and is thus determined recursively by the change of taxes and the ratio of pensioners to workers $R Q_{t}$. Together with the pension budget constraint, the benefit level $\rho_{t}$ and the contribution rate $\tau_{t}^{p}$ are thus determined jointly. $\alpha^{p}$ is a sensitivity parameter reducing the pension benefit level $\rho_{t}$ when the ratio of pensioners to workers $R Q_{t}$ increases, and $\iota$ captures the share of private contributions from wages to a funded pillar of the system. In accordance with legislation we set $\alpha^{p}=0.25$ and $\iota=0.04 .^{29}$

\subsubsection{Health Care System}

We extract a relative age profile of health care expenditures from data by the German public health insurance for years 2010 to $2017 .{ }^{30}$ We normalize this data by GDP taking out time

\footnotetext{
${ }^{29}$ The formula is an approximation to actual legislation, see Ludwig and Reiter (2010).

${ }^{30}$ We thank Friedrich Breyer for sending us the data.
} 
effects and compute the average profile. Next, we hold constant these relative expenditure profiles and feed into the model a time series of average health care contribution rates. For years 1960 to 2012 (again prior to the start of our main experiment) we take the series of contribution rates and the endogenously determined incomes to compute total contributions to health insurance. For our predictions beyond year 2012 we hold constant the age profile of expenditures of the health care system and adjust the contribution rate to clear the budget of the health care system. The details are provided in Appendix C.4.

\section{Quantitative Analysis of the Migration Wave}

Recall from our description of the previous section that we take as given the exogenous population dynamics. As our baseline we consider a counter-factual scenario where the high migration inflow from nationality groups as and $r w$ is absent. The second scenario is the high refugee migration scenario which additionally adds to the demographic dynamics the immigration wave of the years 2013-2018 by the population of asylum seekers. Finally, the high migration scenario adds higher migration numbers from the rest of the world population in the same years on top of the high refugee migration scenario. In all scenarios we assume trend reversals of the migration numbers to their respective long-run averages by 2022 .

\subsection{Population Dynamics}

Figure 4 shows in Panel (a) the sum of net immigration in our three demographic scenarios. ${ }^{31}$ At the peak of the refugee wave in 2015, aggregate migration increased to 1.2 Million in the data (as well as in our high migration scenario). As a consequence of this inflow, the German population increases to about 84 million in 2022 and then starts to decrease. In our high migration scenario the population decreases to 77 million in 2070, and thus relative to the baseline demographic model the size of the total population is about $6 \%$ higher. In Appendix D, Figure 19, we further show the fraction of the population originating from all four regions. By 2070 in the high migration scenario the fraction of natives decreases from $88 \%$ in 2010 to $81 \%$ and the fraction of nationality ho increases from $9 \%$ to $13 \%$. Part of this increase is a consequence of our assumed assimilation from $r w$ to ho, with an assimilation speed of $\pi^{r h}$ of $6 \%$. Correspondingly, the fraction from $r w$ first increases, then decreases. Finally, the fraction of asylum seekers in the population increases from $2 \%$ to $5 \%$.

\footnotetext{
${ }^{31}$ In the baseline scenario, net immigration to Germany is negative in 2016. The reason is an exceptionally high net emigration of German natives. The long-run average emigration of natives is small, around -23,000. In our projections, we abstract from emigration of all groups.
} 
Figure 4: Migration \& Total population

(a) Total Net Migration

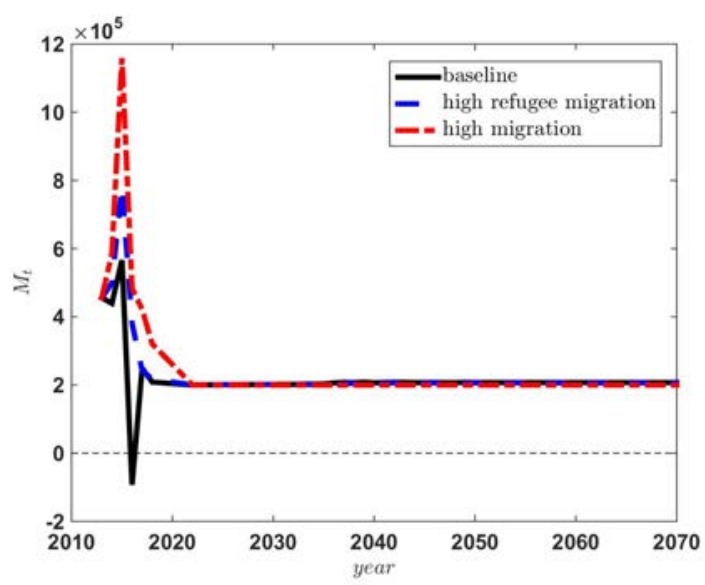

(b) Total population

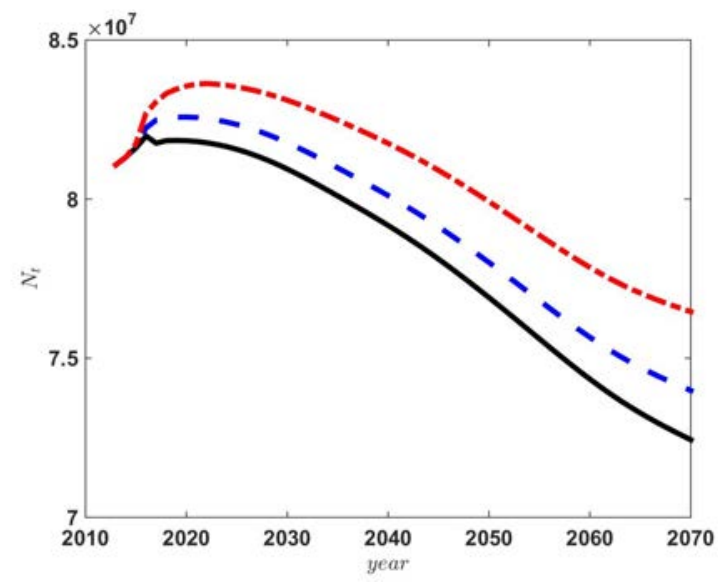

Notes: Panel (a): Total net immigration; Panel (b): total German population in three migration scenarios.

Figure 5 plots the working age to population ratio, the fraction of the population aged $j_{a}=$ 17 to $j_{r l}-1=66$ to the total population, as well as the old-age dependency ratio, defined as the fraction of the population aged $j_{r l}=66$ to $J=100$ to the working-age population. These plots underscore that our analysis of the migration wave occurs against the backdrop of a massively aging population. In the baseline demographic scenario, the old-age dependency ratio increases from $30 \%$ in 2010 to $50 \%$ by 2050, and the working-age to population ratio falls from $65 \%$ to $55 \%$. The inflow of young immigrants has substantial effects, in particular on the old-age dependency ratio. It decreases by 1.5\% until 2040 in the high migration scenario. The figure also shows the effects of the boom-bust nature of our migration experiment. Towards 2050-60 the young migrants of the 2015's start to retire, which then decreases the working age to population ratio sand increases the old-age dependency ratio.

\subsection{Macroeconomic and Welfare Effects}

As our baseline we study a closed economy population in which aging and the inflow of migrants affect not only relative prices of labor of different population groups, but also total factor prices as in the simple model of Section 4. Since Germany is by no means a completely closed economy, in Section 7.3 we also report results for a small open economy version of the model. For each of the three population scenarios, we hold constant government expenditures per refugee, and government consumption expenditures are held constant at the path computed in the baseline demographic model. The government budget is cleared through adjusting consumption taxes. 
Figure 5: Dependency Ratios

(a) WAPR

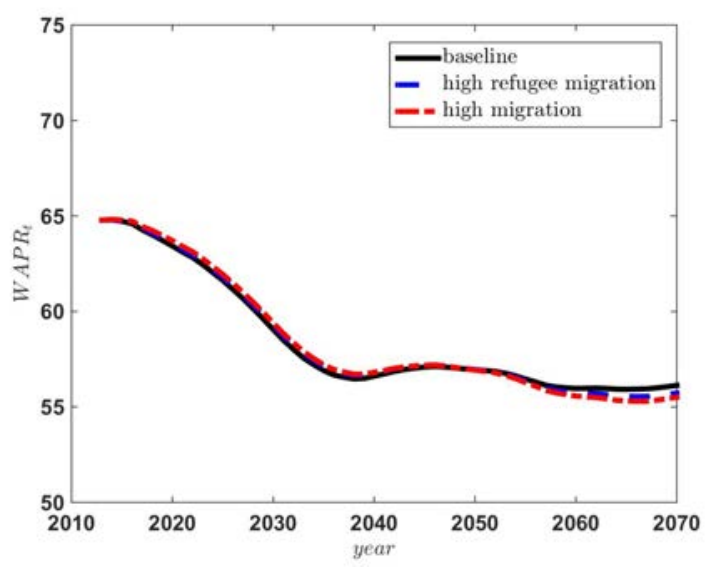

(c) OADR

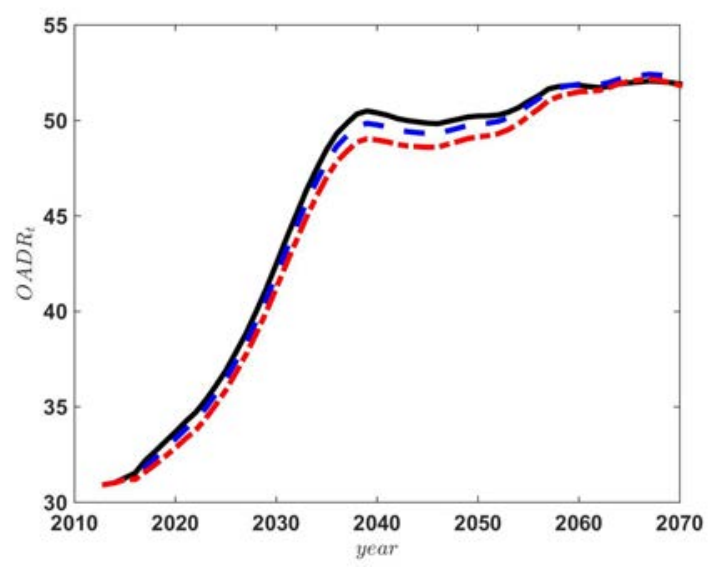

(b) \%p-Change of WAPR

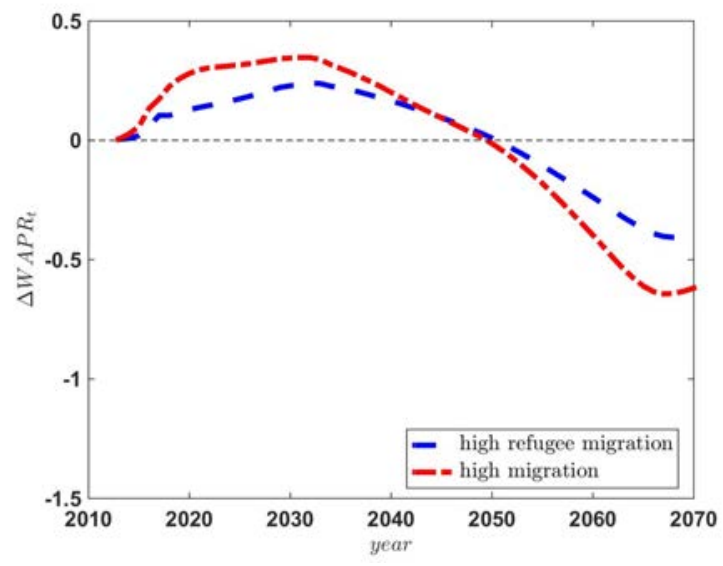

(d) \%p-Change of OADR

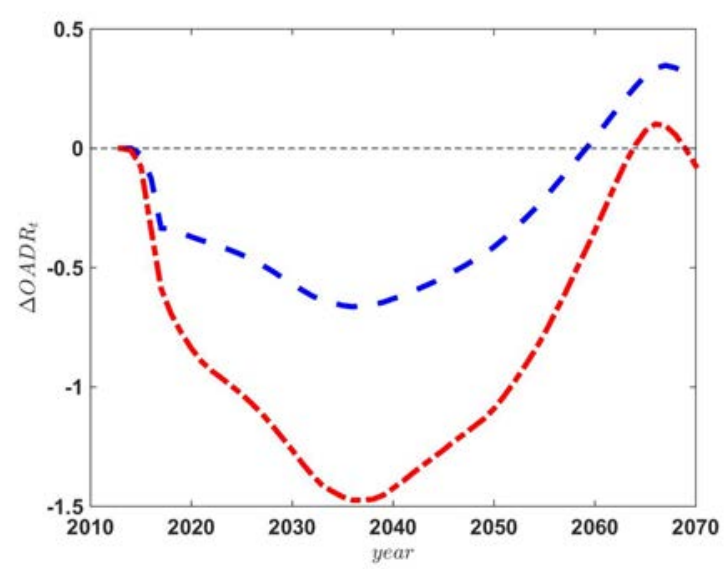

Notes: Panel (a): working age to population ratio, Panel (b): percentage point change of working age to population ratio; Panel (c): old-age dependency ratio; Panel (d): percentage point change of old-age dependency ratio.

\subsubsection{Social Security System}

The fiscal consequences of the migration flow on the pension system are shown in Figure 6. As a consequence of population aging the contribution rate in the pension system increases from $20 \%$ in 2010 to $25 \%$ by 2040 . Correspondingly, the benefit level falls by 4 percentage points. The migration inflow leads, in the high migration scenario, to a reduction of the contribution rate by up to $0.2 \% \mathrm{p}$ and an increase of the benefit rate also by about $0.2 \% p$ in 2030. As for the working age to population and the old-age dependency ratios, the figure shows the reversal of these changes after 2060 when the young migrants retire. Appendix D.2 complements these findings by displaying expenditures of the health care system. 
Figure 6: Contribution \& Replacement Level

(a) Contribution Rate $\tau_{t}^{p}$

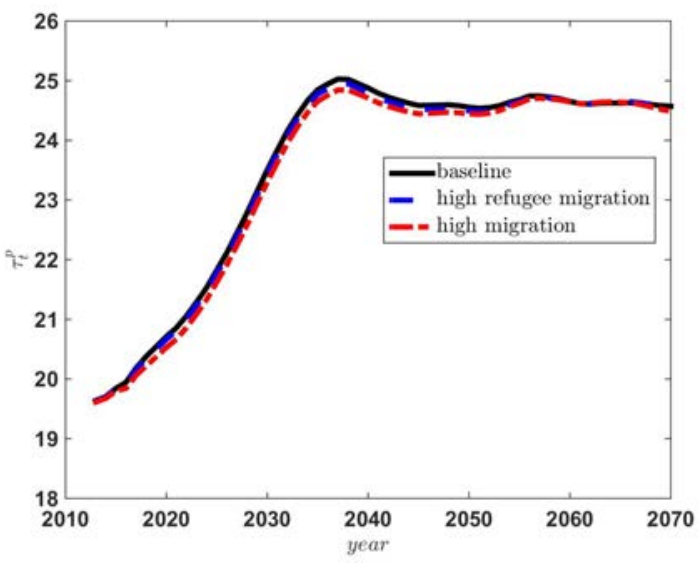

(c) Benefit Rate $\rho_{t}^{p}$

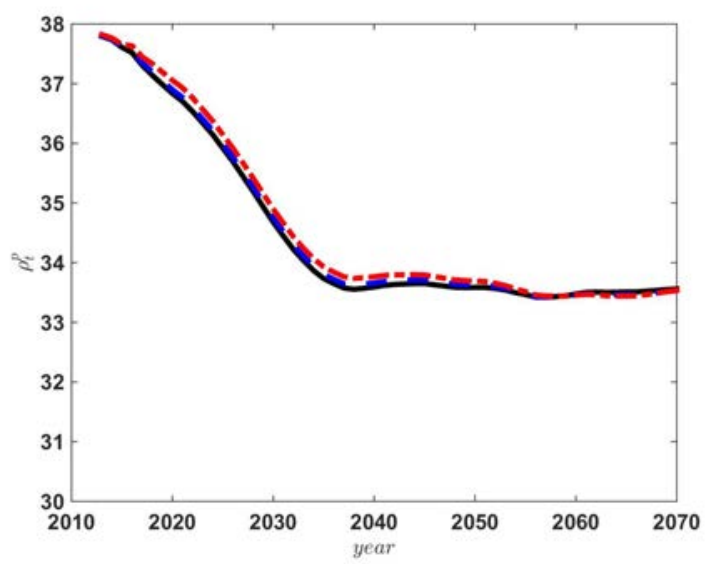

(b) \%p-Change of Contribution Rate

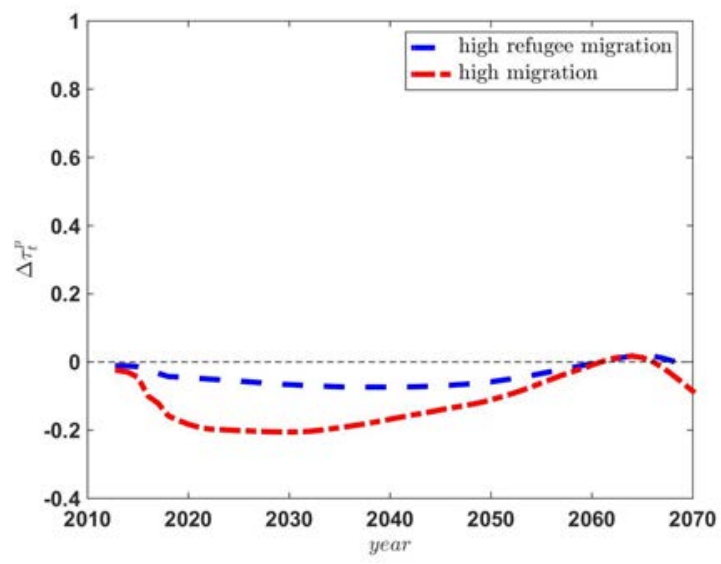

(d) \%p-Change of Benefit Rate

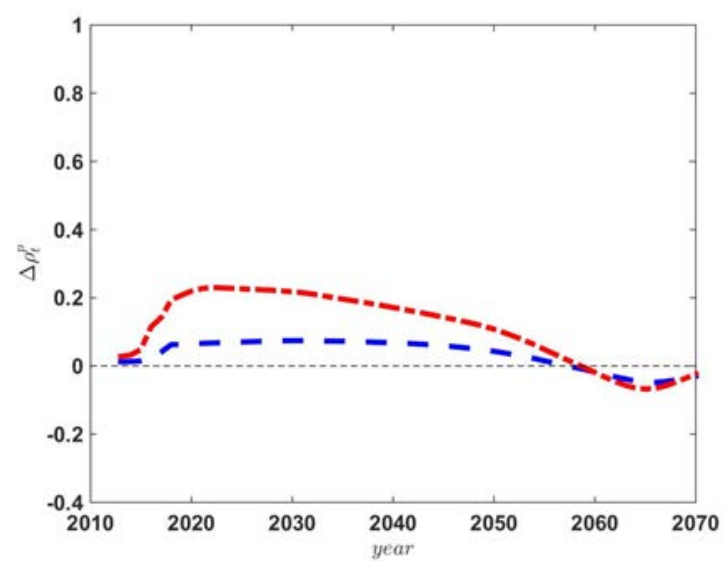

Notes: Panel (a): contribution rate to pension system, Panel (b): percentage point change of contribution rate to pension system; Panel (c): benefit rate of pension system; Panel (d): percentage point change of benefit rate of pension system.

\subsubsection{Government Expenditures and Consumption Taxes}

Because of the administrative outlays on refugees, the total government expenditure to GDP ratio increases after the migration shock, see Appendix D.2. In the long-run, however, since general government consumption expenditures $G_{t}$ do not adjust to the flow of incoming people and GDP increases due to the increasing workforce, the ratio of total government expenditures to GDP decreases. Consumption taxes shown in Figure 7 adjust to clear the government budget. In the baseline demographic model, as a consequence of the lower workforce which shrinks the tax base and the constant government consumption expenditures as a share of GDP, the consumption tax rate needs to increase by almost $8 \% \mathrm{p}$ along the transition. Financing administrative expenses for the incoming refugees leads to an initial 
spike in the consumption tax rate (in the refugee migration scenario). Over time, however, consumption taxes decrease in the two migration scenarios because of the enlarged tax base.

Figure 7: Consumption Taxes

(a) Consumption Tax Rate $\tau_{t}^{c}$

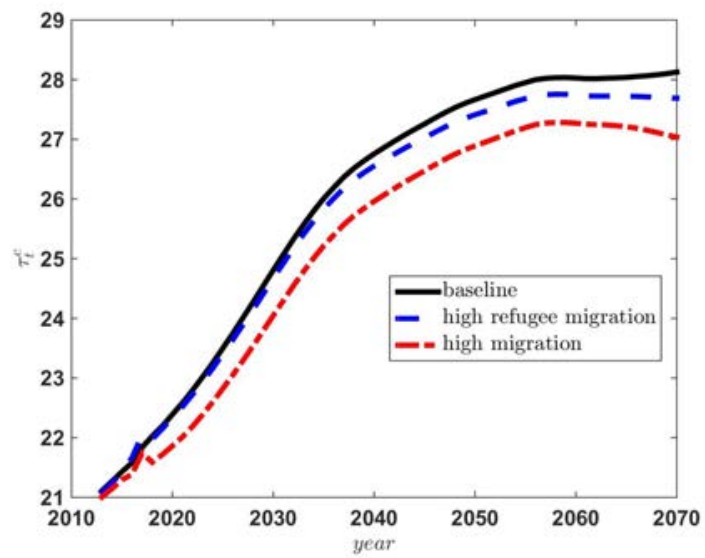

(b) \%p-Change of Cons. Tax Rate

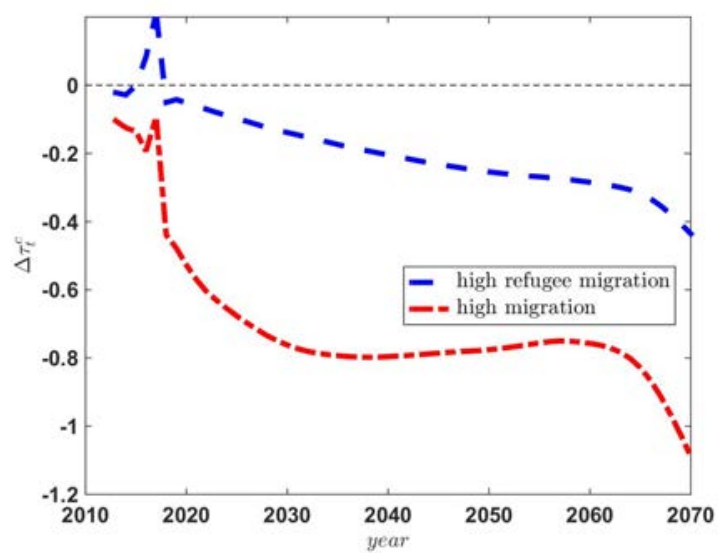

Notes: Panel (a): consumption tax rate, Panel (b): percentage point change of consumption tax rate.

\subsubsection{Macroeconomic Aggregates}

We de-trend per capita GDP and per capita consumption by the technology level in order to isolate the effects of demographic changes. Thus if these variables decline, this means they fall relative to a constant trend growth. In the baseline, detrended per capita GDP and consumption decrease along the transition of the economy as shown in Figure 23 of Appendix D. The immigration of mainly low-skilled individuals leads to a drop in both variables because they add little to output, relative to their head count. However, to draw conclusions about the welfare consequences of such an inflow for those who live along the transition, in addition to analyzing its fiscal consequences we have to study the impact on rates of return and wages as the relevant ingredients for household life-cycle decisions and utility. ${ }^{32}$ We turn to this analysis next.

\subsubsection{Wages}

Marginal Product of Labor. Figure 8 shows the marginal product of labor $w_{t}$. In the baseline scenario, the marginal product of labor increases between 2013 and 2035 by almost $3 \%$ on account of the aging population and associated decline in labor relative to

\footnotetext{
${ }^{32}$ Krueger and Ludwig (2007) make the related point that for evaluating the welfare consequences of demographic change the time paths of per capita consumption and output alone are not fully informative.
} 
capital, and then swings back. ${ }^{33}$ Comparing now the baseline and the high migration scenarios, as a consequence of the inflow of immigrants the marginal product of labor drops on impact by more than 0.4 percent. In our model, low skilled immigrants arrive without any assets and thus the per capita capital stock declines. At the same time aggregate labor increases mildly. Both forces reduce the capital stock per labor efficiency unit and thus the marginal product of labor, while raising the rate of return to capital. The reduction of the marginal product of labor has adverse welfare consequences for young agents, the increase of the rate of return in contrast is beneficial for medium- and old-aged native households alive in 2013 who have substantial asset holdings. Importantly, after the initial drop the gap between the marginal product of labor in the high refugee migration and the high migration scenarios gradually closes until 2035 as the young immigrants accumulate wealth so that the capital-labor ratio (capital intensity) $k_{t}$ increases.

Figure 8: Marginal Product of Labor

(a) MPL

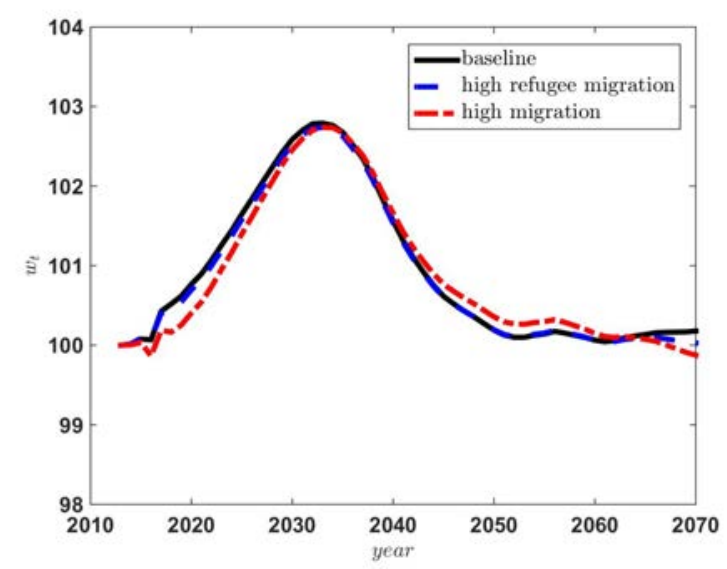

(b) \%-Change of MPL

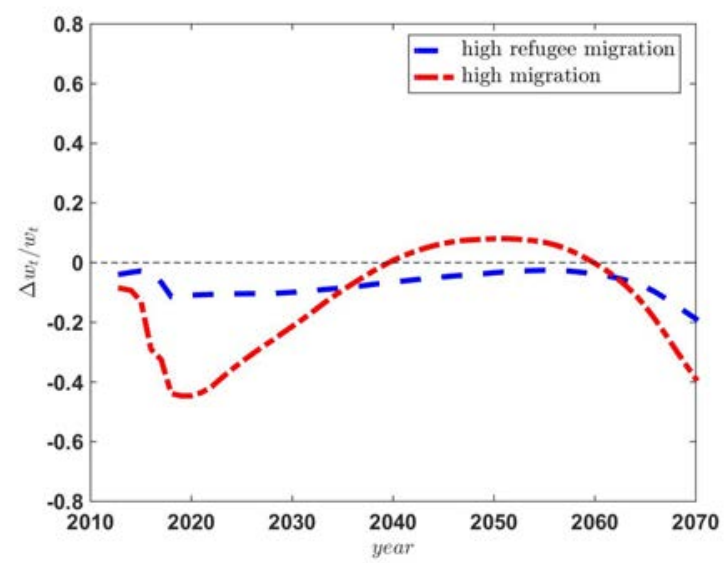

Notes: Panel (a): marginal product of labor, Panel (b): percent change of marginal product of labor.

Wages of Low-Skilled Natives. We now focus on low-skilled natives who make up 7.4\% of the native working age population in 2013. ${ }^{34}$ Figure 9 shows the percent changes of aggregate gross and net wages of low-skilled natives in the first age (experience) group $\bar{j}=1$, $w_{t}(\bar{j}=1, s, i)$ for $s=l o, i=n a$, whereas the corresponding levels are shown in Figure 25 in Appendix D. ${ }^{35}$ The migration inflow reduces gross wages of low-skilled natives by $1.5 \%$ in 2040 in the high migration scenario, and throughout the entire projection window the

\footnotetext{
${ }^{33}$ The return on capital $r_{t}$ displays the opposite movement, shown in Figure 24 in Appendix D: it decreases by about $0.5 \%$ p, until 2035, consistent with previous studies for Germany, e.g., Börsch-Supan et al. (2006).

${ }^{34}$ Computed as the weighted (by gender) share of natives with a low education, see Table 3.

${ }^{35}$ Results for other experience groups are similar.
} 
gross wage effect is negative. The net wage reduction is smaller initially in both migration scenarios since pension contribution rates decline, but becomes stronger over time as pension contributions increase with the gradual retirement of the migrant wave.

Figure 9: \%-Changes of Gross \& Net Wages, Low-Skilled Natives, Age Group $\bar{j}=1$

(a) \%-Change of Gross Wages

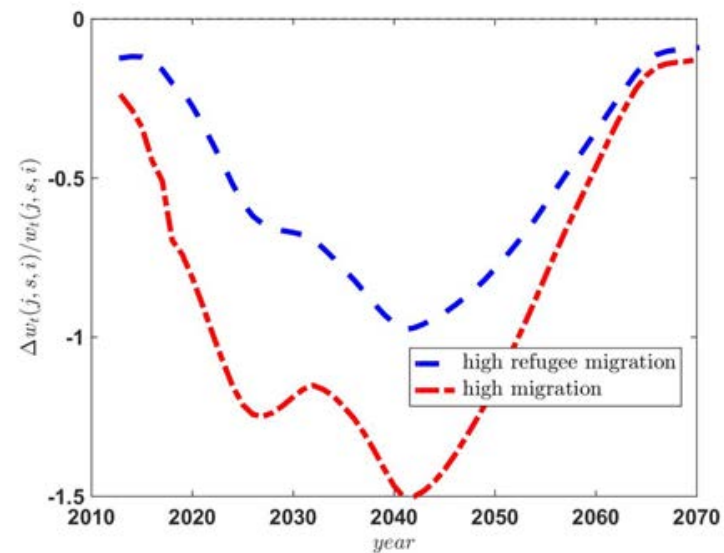

(b) \%-Change of Net Wages

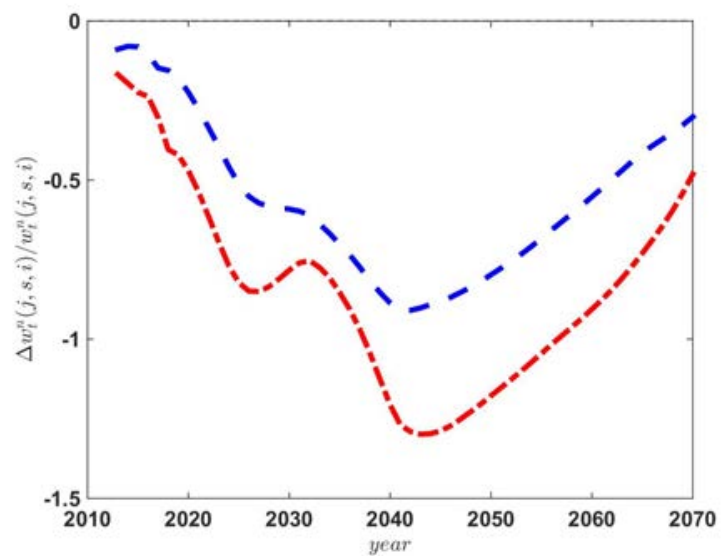

Notes: Panel (a): percent change of gross wages, Panel (b): percent change of net wages of low-skilled natives in age group $\bar{j}=1$.

Decomposition of Gross Wages of Low-Skilled Natives. To interpret the change in gross wages we decompose them into the marginal product of labor and additional terms that reflect the relative scarcity of skills and of native workers, exactly as in the simple model of Section 4. After substitution of the different components of the aggregate production function, and again focusing on wages of low-skilled natives we obtain:

$$
\begin{gathered}
w_{t}(\bar{j}=1, l o, n a)=w_{t} \cdot \underbrace{\left(1+\Theta_{t}(m e, l o)^{1-\frac{1}{\sigma_{l m h}}}+\Theta_{t}(h i, l o)^{1-\frac{1}{\sigma_{l m h}}}\right)^{\frac{1}{\sigma_{l m h}-1}}}_{=\mathcal{W}_{t}^{s}(l o)} . \\
\underbrace{\left(1+\Theta_{t}(1, f o, n a \mid l o)^{\left.1-\frac{1}{\sigma_{n f}}\right)^{\frac{1}{\sigma_{n f}-1}}}\right.}_{=\mathcal{W}_{t}^{f n}(l o)}
\end{gathered}
$$

where $\Theta_{t}(s, l o)=\frac{L_{t}(s)}{L_{t}(l o)}$ for $s \in\{m e, h i\}$ is the ratio of the CES aggregates of workers with skill $s$ to the CES aggregate of low-skilled workers and $\Theta_{t}(1, f o, n a \mid l o)=\frac{\tilde{L}_{t}(1, l o, f o)}{L_{t}(1, l o, n a)}$ is the ratio of the CES aggregate of foreign to the CES aggregate of low-skilled native workers in the youngest age group. These terms measure the relative scarcity of the respective productivity-weighted labor aggregates. They in turn enter into expressions for the relative 
wage effects. We refer to $\mathcal{W}_{t}^{s}(s)$ as the relative scarcity wage effect of skill group $s$, and to $\mathcal{W}_{t}^{f n}(s)$ as the relative scarcity wage effect of foreign workers of skill group s.

Our estimates of the substitution elasticities $1<\sigma_{l m h}<\infty$ and $1<\sigma_{n f}<\infty$ from Section 6 suggest that workers with different education levels and of different nationalities are imperfect substitutes. Then three effects from an inflow of low skilled immigrants on low-skilled natives' wages are at work, as in the simple model of Section 4. First, an inflow of workers with zero assets decreases the capital stock per capita and increases aggregate labor supply, thus reducing the capital intensity $k_{t}$ which decreases gross wages $w_{t}$. Second, an inflow of low-skilled foreign workers makes low-skilled natives scarcer, i.e. it increases the skill ratio $\Theta_{t}\left(1, f_{o}, n a \mid l o\right)$, which increases gross wages of native low skilled workers. Third, an increase of low-skilled workers increases the relative scarcity of medium and high skilled workers relative to low skilled workers, i.e., it decreases the skill ratios $\Theta_{t}(s, l o)$, for $s \in\{m e, h i\}$, which decreases wages of (native) low skilled workers.

Figure 26 in Appendix D displays the changes in the skill ratios $\Theta_{t}(\cdot)$. Due to the inflow of relatively low-skilled workers, high- and medium-skilled workers become relatively scarce. Since the skill decomposition of refugees and of foreigners from RW features stronger differences to natives in the medium skill group than among the high-skilled, this scarcity effect is more pronounced for $\Theta_{t}(m e, l o)$ than for $\Theta_{t}(h i, l o)$. This increase of the relative abundance of low skilled workers contributes to a reduction of gross wages. The effect is stronger than the increase of the relative scarcity of natives to foreigners of low skill, shown by term $\Theta_{t}(f o, n a \mid l o)$.

How these relative scarcities of workers translate into wage effects also depends on the respective substitution elasticities. The total effect is measured by the relative scarcity terms $\mathcal{W}_{t}^{s}(l o)$ and $\mathcal{W}_{t}^{f n}(l o)$; their changes are shown in Figure 10 . As a consequence of the strong changes of the skill ratios shown in Figure 26 and of the low estimate of the elasticity of substitution across skill groups, the wage effect due to the relative abundance of low skilled workers is substantially more negative than the positive effect from the relative scarcity of native workers. This, in combination with the reduction of the marginal product of labor shown in Figure 8 explains the reduction of gross wages shown in Figure 9.

\subsubsection{Distribution of Welfare Changes}

To analyze the welfare consequences of the different population scenarios we compute consumption equivalent variation $(\mathrm{CEV})$ between the high refugee migration scenario and the baseline scenario, as well as between the high migration scenario and the baseline scenario. The CEV is computed as the percent change in consumption over the life-cycle required as compensation in the baseline scenario for individuals to be indifferent to the respective mi- 
Figure 10: Change of Wage Terms, Low-Skilled Natives, Age Group $\bar{j}=1$

(a) $\Delta \mathcal{W}_{t}^{s}(l o) / \mathcal{W}_{t}^{s}(l o)$

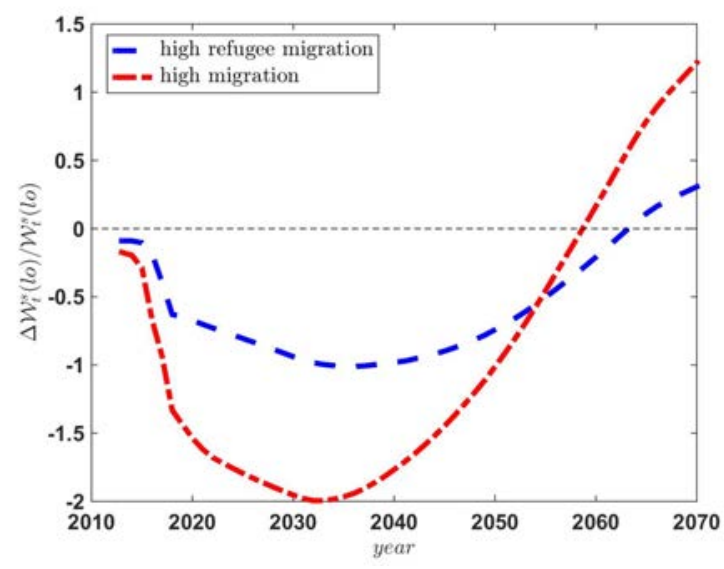

(b) $\Delta \mathcal{W}_{t}^{f n}(l o) / \mathcal{W}_{t}^{f n}(l o)$

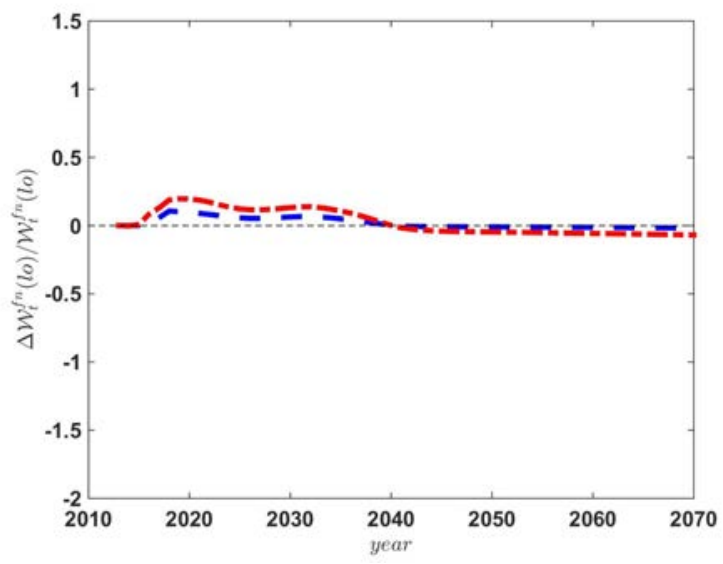

Notes: Decomposition of wages according to equation (46). Panel (a): relative scarcity wage effect of skill group lo, Panel (b): relative scarcity wage effect of foreign workers of skill group $l o$.

gration scenario. Thus, positive numbers indicate welfare gains from a migration scenario. For every cohort that lives at any point in time during the studied period, we calculate the CEV based on discounted life-time utility. Thus, in 2013 we compute the CEV for all ages $j=0, \ldots, J$, reflecting all cohorts alive in 2013. For all cohorts born after 2013 we calculate it at the initial condition $(j=0, a=0)$ of the newborns. For each cohort, we calculate CEVs separately by skill group $s$ and nationality $i$.

Panels (a) and (b) of Figure 11 show the CEV of native cohorts alive in 2013 for all skill groups. In the refugee migration scenario, the medium- and high-skilled gain whereas the low skilled lose from the migration inflow. The highest gains of $0.2 \%$ of consumption equivalent variation are obtained by medium and high skilled natives at labor market entry, and highest losses of $-0.2 \%$ accrue to low-skilled natives of about age 35. As Panels (c) and (d) show, in contrast to the very mild initial welfare losses faced by low-skilled natives, future cohorts increasingly gain from the in-migration of low-skilled refugees because the positive effects from the relatively young age distribution dominate the negative wage effects. ${ }^{36}$ Uncovering this heterogeneity in the welfare consequences is an important advantage of our dynamic analysis, relative to static investigations that focus on the very short-run or the long-run steady state.

Since low-skilled natives only make up $7.4 \%$ of the native population, and since the high and medium skilled are winners from the inflow of predominantly low-skilled migrantsboth in the 2013 cross-section as well as for newborns in many years along the transition,

\footnotetext{
${ }^{36}$ The mild losses for newborn low skilled natives are in 2013-2107, respectively, $-0.0568 \%,-0.0464 \%,-0.0365 \%,-0.0267 \%$ and $-0.0145 \%$.
} 
Figure 11: Consumption Equivalent Variation (CEV) for Natives [in \%]

(a) Generations Alive in 2013, Refugee Migration

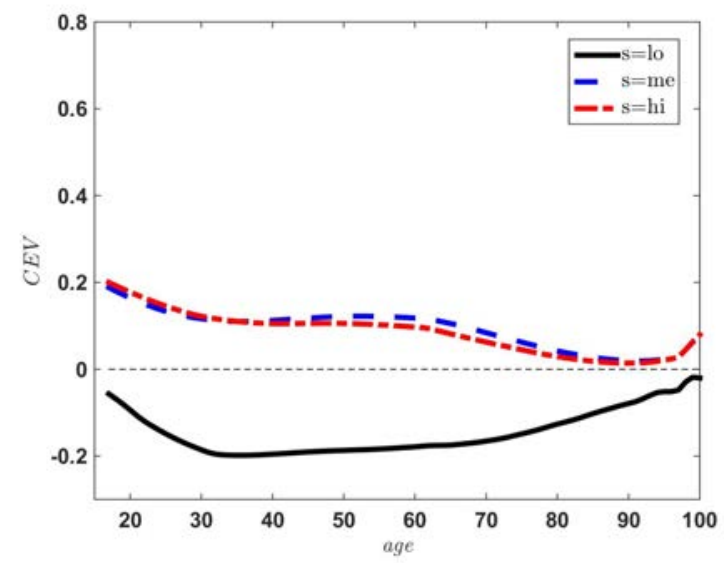

(c) By Birth Cohort over Time, Refugee Migration

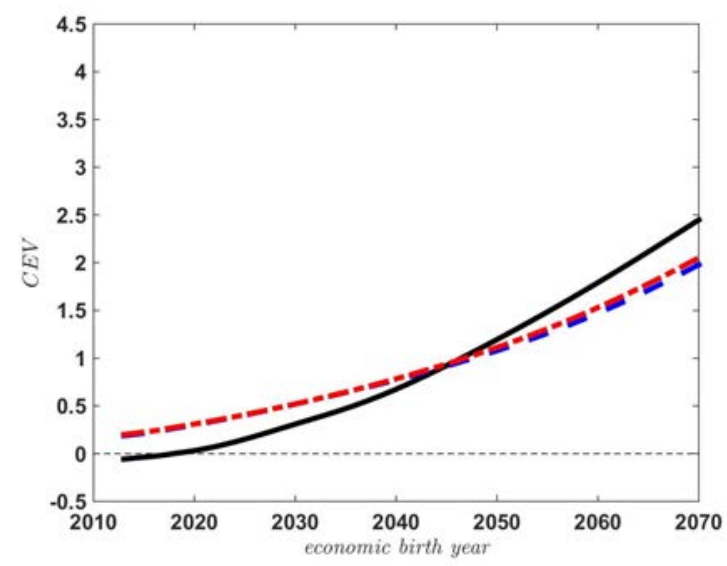

(b) Generations Alive in 2013, High Migration

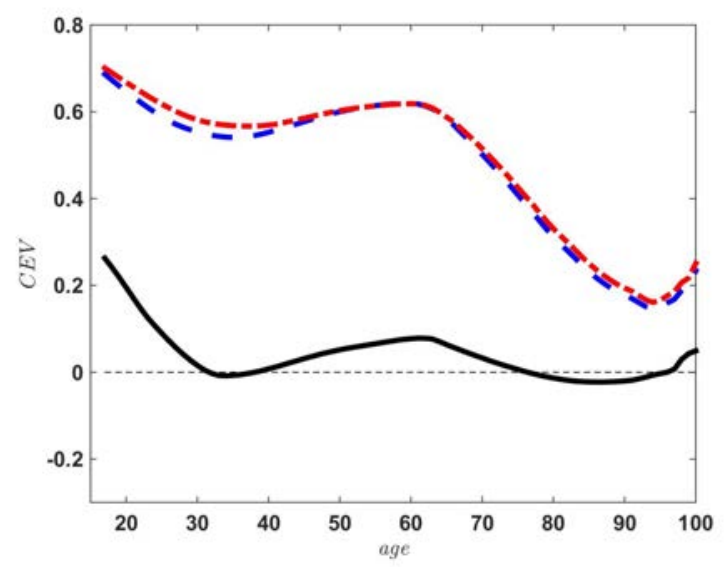

(d) By Birth Cohort over Time, High Migration

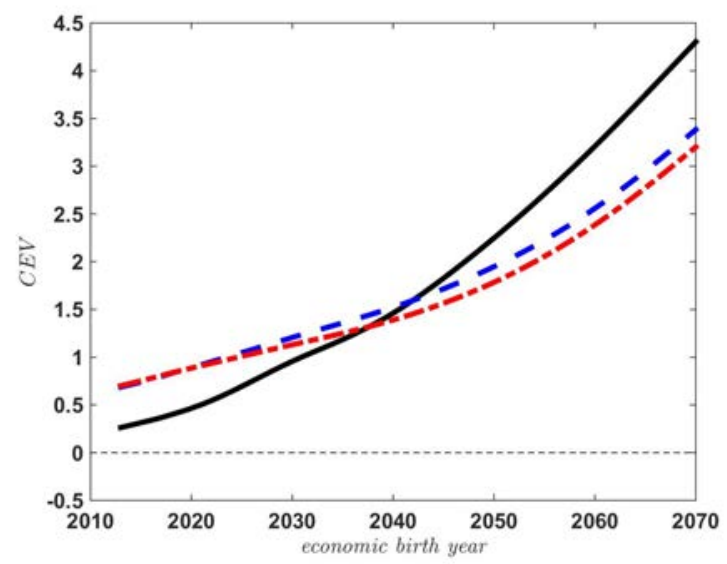

Notes: Panel (a): consumption equivalent variation (CEV) of natives alive in 2013 over age in refugee migration scenario, Panel (b): CEV of natives alive in 2013 over age in high migration scenario, Panel (c): $\mathrm{CEV}$ of economically newborns over time in refugee migration scenario, Panel (d): CEV of economically newborns over time in high migration scenario.

as shown in Figure 11-, the fraction of winners by far exceeds the fraction of losers among the natives. The larger fraction of winners is also income and consumption richer than the losers. Even though the gains expressed in terms of a consumption equivalent variation of the winners are mostly smaller than the losses of the natives, the higher overall economic weight of the winners suggests that there is room for a cross-sectional redistribution scheme within the native population to compensate the losers.

To see the potential for such a cross-sectional redistribution scheme in each year, we compute the aggregate gains (or losses) based on the CEVs of all households alive in a given year $t$. For this calculation, we first translate the CEV's into year $t$ consumption units for 
all population groups alive in that year. Aggregation then gives a measure of total gains (or losses) in the year $t$ cross-section, which we relate to year $t$ aggregate private consumption in the baseline. Concretely, denote by $g_{t-j}^{c}(s, i)$ the education $s$, nationality $i$, and cohort $(t-j)$ specific CEV and by $c_{t}(j, s, i)$ the respective average consumption (averaged across endogenous asset and employment distributions and the exogenous gender distribution) in the baseline scenario. The period $t$ net gain of group $j, s, i=n a$ is determined as

$$
X_{t}(j, s, i=n a)=N_{t}(j, s, i=n a) \cdot g_{t-j}^{c}(s, i=n a) \cdot c_{t}(j, s, i=n a)
$$

where $N_{t}(j, s, i=n a)$ is the size of the age $j$, education $s$ native population in year $t$. Next, for each year $t$ we sum up the obtained gains and losses for the cross-section of all natives living in a given year to obtain the net gain expressed in units of consumption of that year. We then express these net gains as a fraction of aggregate private consumption of natives in the baseline scenario:

$$
\mathcal{X}_{t}=\frac{\sum_{j=0}^{J} \sum_{s \in\{l o, m e, h i\}} X_{t}(j, s, i=n a)}{C_{t}(i=n a)}
$$

In the cross-section of households alive in 2013 the net gain ratio in the refugee migration scenario is small but positive: it stands at $0.087 \%$. In the high migration scenario it is $0.51 \%$. Figure 12 displays the net gain ratio for years 2013-2070. In the refugee migration scenario while the net gain ratio is initially small it increases over time and reaches $0.6 \%$ by 2070 .

Figure 12: Net Gain Ratio [in \%]

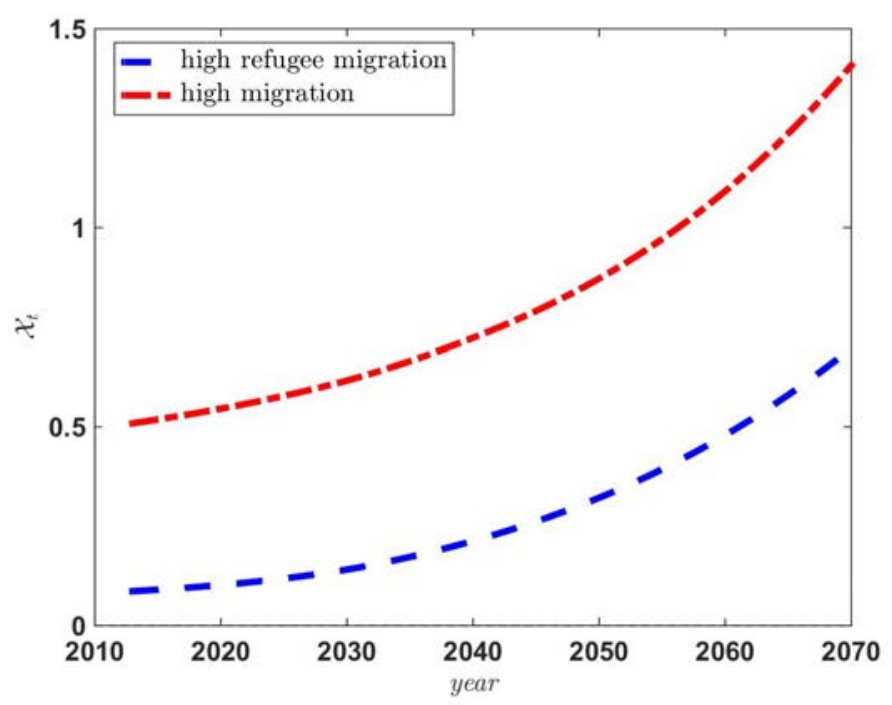

Notes: Net gain ratio for the cross-section of a given period as defined in equation (48) over time. 
Overall, in the refugee migration scenario the aggregate welfare consequences and effects summarized in the net gain ratio are relatively small initially, partially due to the fact that the inflows are not massive, relative to the size of the native workforce. However, they increase over time and the aggregate perspective masks the distributional implications highlighted above, with currently alive low-skilled natives experiencing losses whereas native medium- and high-skilled households experience substantial welfare gains from the inflow, which overall nets to a small but positive number in 2013 in the aggregate.

\subsection{Sensitivity Analyses}

We investigate the sensitivity of our results by analyzing how the CEV of low-skilled native newborns in 2013 and the net gain ratio in the 2013 cross-section are affected by changes in the economic environment. The results are summarized in Table 4. First, and motivated by our simple model of Section 4 we assume a small open economy. In our experiment, we hold interest rates constant from year 2013 onwards, effectively assuming that the economy opens up to international capital flows from then on, modeled as a zero probability event in all migration scenarios. As a consequence and in line with the predictions from the simple model of Section 4, the CEV of economic newborns improves relative to the closed economy model; it is now approximately zero in the refugee migration scenario. Likewise, the net gain ratio increases. Given that the German economy is a medium sized open economy our baseline results for the closed economy may therefore overstate the welfare losses from low-skilled immigration for low-skilled natives.

Next, we investigate sensitivity with respect to some key parameter estimates. We consider an increase of the assimilation probability of asylum seekers from $\pi^{a r}=0.008$ to the speed at which migrants from $r w$ assimilate to $h o$ of $\pi^{r h}=0.06$. We view the assimilation speed to some degree as a policy parameter that may be changed by relaxing labor market frictions or labor market training for asylum seekers. There are two countervailing mechanisms. On the one hand, a higher assimilation speed increases the labor market competition for low skilled natives. On the other hand, a higher assimilation speed increases the overall economic benefits from low-skilled immigration, enlarging the tax base, which has positive welfare effects also for low skilled natives. In the refugee migration scenario the net effect leads to slightly higher welfare losses for low-skilled natives. In the high migration scenario, the gains dominate for this group. In both scenarios the net gain ratio increases.

Finally, we evaluate the sensitivity with respect to our parameter estimates of the substitution elasticities in production. Specifically, we explore a perfect substitution elasticity (PSE) within foreigners and between foreigners and natives by setting $\sigma_{h r}=\sigma_{n f}=\infty$, 
labeled as "Nationality-PSE". This increases labor market competition from the inflow of low-skilled workers for low-skilled natives and for all other native workers. Accordingly, the CEV of low skilled natives is more strongly negative than in the baseline model, and the net gain ratio decreases. Related, we also analyze the effects of a perfect substitution across skill groups by setting $\sigma_{l m h}=\infty$, labelled as "Skills-PSE". In this scenario, labor market competition from the inflow of low-skilled migrants for low-skilled natives is less severe, but for the medium and high skilled it increases. Accordingly, the CEV for the low-skilled natives increases substantially and turns positive in the refugee migration scenario. The net gain ratio decreases mildly because of the intensified labor market competition for medium and high skilled natives.

Table 4: CEV of Low-Skilled Native Newborns in 2013 and 2013 Net Gain Ratio [in \%]

\begin{tabular}{lcccc}
\hline \hline & \multicolumn{2}{c}{ CEV 2013 } & Newborns [in \%] & 2013 Net Gain Ratio [in \%] \\
\hline Variant & Ref Scen & High Mig Scen & Ref Scen & High Mig Scen \\
\hline Baseline & -0.0568 & 0.2628 & 0.0868 & 0.5093 \\
\hline SOE & 0.0001 & 0.4805 & 0.1281 & 0.6901 \\
$\pi^{a r}=0.06$ & -0.0595 & 0.3173 & 0.2288 & 0.6636 \\
Nationality-PSE & -0.2316 & -0.05 & 0.0806 & 0.4844 \\
Skills-PSE & 0.2959 & 0.8533 & 0.0764 & 0.4955 \\
\hline \hline
\end{tabular}

Notes: CEV of low-skilled natives economically born in 2013 and net gain ratio for the 2013 cross-section as defined in equation (48).

\section{Conclusion}

To study the macroeconomic and distributional consequences of the recent migration wave to Germany we have constructed a quantitative overlapping generations economy with skill heterogeneity among the German population. We found that net wages of unskilled natives deteriorate in the short run as an increased number of unskilled refugees compete with these natives on the labor market and as administrative expenses on (mainly) low skilled immigration increase. We also document that these welfare losses are dominated by the gains for other population groups. Our results point to general lessons that apply beyond the German experience. First, for the wage and welfare consequences of different groups it is crucial what skill segment the migrants belong to and compete with, and the binary distinction between college and non-college educated workers might not be granular enough in many countries. Related, the relative magnitude of the substitution elasticities across worker skill types, and across regions of origin of workers are crucial determinants in the 
wage- and thus welfare consequences for natives of large migration flows. Third, the welfare consequences of migration for natives are not only heterogeneous by education and wealth (highlighting the potential importance of endogenous interest movements in the presence of massive shifts in the supply of labor), but vary substantially over time and across cohorts, requiring the type of dynamic transition analysis in the context of an OLG model presented here. Fourth, even though the aggregate welfare consequences of these migration waves might be small, at least initially, they mask very sizeable redistribution across groups exactly along the dimensions discussed above, with substantial transfers required to compensate the losers. And finally, over time, welfare effects become more and more positive to the effect that also low skilled native newborns benefit from the inflow of low skilled workers, because in an aging society the favorable (relatively young) age distribution of the immigrants dominate the relatively low productivity effects eventually.

We have abstracted from a number of aspects when modeling migrant inflow and behaviour that might be quantitatively important for our conclusions. First, by assuming a constant returns to scale production function we abstract from the existence of fixed factors such as land or housing and the associated negative congestion effects induced by increased migration. Second, by focusing on formal labor market outcomes we likely have underestimated the economic contributions of migrant labor to the German economy, for example, when migrants engage in black market activities or contribute (unmeasured) labor to businesses in their social networks. Finally, even though we have modelled skill assimilation of migrants, this was not driven by choice (conscious human capital accumulation), but by chance. Similarly, although we have considered the possibility of return migration as an exogenous stochastic event, especially economic migrants and successful asylum seekers face a choice of whether to remain in Germany, and if so, how long. Although our focus has been on the outcome for native Germans, an equally important question concerns the economic consequences for the migrants themselves, conditional on the assumption that the decision to leave their home countries was driven by exogenous shocks (in the case of Syrian refugees, the civil war). However, addressing this question would require modeling the economic future of the migrants' home country, which is a daunting task especially in the case of the current refugees. We therefore leave this question to future research. 


\section{References}

Auerbach, A. J. and L. J. Kotlikoff (1987). Dynamic Fiscal Policy. Cambridge, MA: Cambridge University Press.

Battisti, M., G. Felbermayr, G. Peri, and P. Poutvaara (2017, 11). Immigration, Search and Redistribution: A Quantitative Assessment of Native Welfare. Journal of the European Economic Association 16(4), 1137-1188.

Ben-Gad, M. (2004, July). The Economic Effects of Immigration-a dynamic Analysis. Journal of Economic Dynamics and Control 28(9), 1825-1845.

Ben-Gad, M. (2008, April). Capital-Skill Complementarity and the Immigration Surplus. Review of Economic Dynamics 11(2), 335-365.

Benabou, R. (2002). Tax and Education Policy in a Heterogeneous-Agent Economy: What Levels of Redistribution Maximize Growth and Efficiency? Econometrica 70(2), 481517.

Borjas, G. (1985). Assimilation, Changes in Cohort Quality, and the Earnings of Immigrants. Journal of Labor Economics 3(4), 463-489.

Borjas, G. J. (1999). The Economic Analysis of Immigration. In O. Ashenfelter and D. Card (Eds.), Handbook of Labor Economics, Volume 3A, Chapter 28, pp. 16791760. Amsterdam: Elsevier.

Borjas, G. J. (2003). The Labor Demand Curve is Downward Sloping: Reexamining the Impact of Immigration on the Labor Market. Quarterly Journal of Economics 118(4), 1335-1374.

Börsch-Supan, A., A. Ludwig, and J. Winter (2006). Aging, Pension Reform and Capital Flows: A Multi-Country Simulation Model. Economica 73, 625-658.

Brell, C., C. Dustmann, and I. Preston (2020). The Labor Market Immigration of Refugee Migrants in High-Income Countries. CReAM Discussion Paper Series (01/20).

Brücker, H. and E. J. Jahn (2011). Migration and Wage-setting: Reassessing the Labor Market Effects of Migration. The Scandinavian Journal of Economics 113(2), 286-317.

Burstein, A., G. Hanson, L. Tian, and J. Vogel (2017). Tradability and the Labor-Market Impact of Immigration: Theory and Evidence from the U.S. NBER Working Paper.

Chassamboulli, A. and T. Palivos (2014). A Search-Equilibrium Approach to the Effects of Immigration on Labor Market Outcomes. International Economic Review 55(1), $111-129$. 
Chojnicki, X., F. Docquier, and L. Ragot (2011). Should the US Have Locked Heaven's Door? Journal of Population Economics 24(1), 317-359.

Colas, M. (2019). Dynamic Responses to Immigration. Opportunity and Inclusive Growth Institute.

Czerny, L. (2019). Essays on Migration, Public Spending and Local Fiscal Multipliers. Dissertation, Goethe University Frankfurt.

D'Amuri, F., G. I. Ottaviano, and G. Peri (2010, May). The Labor Market Impact of Immigration in Western Germany in the 1990s. European Economic Review 54(4), $550-570$.

d'Artis Kancs and P. Lecca (2018). Long-term Social, Economic and Fiscal Effects of Immigration into the EU: The Role of the Integration Policy. World Economy.

Diamond, P. A. (1965). National Debt in a Neoclassical Growth Model. American Economic Review 55, 1126-1150.

Dustmann, C. and I. Preston (2012, 02). Comment: Estimating The Effect of Immigration on Wages.

Dustmann, C., U. Schonberg, and J. Stuhler (2016). The Impact of Immigration: Why do Studies reach such different Results? Journal of Economic Perspectives 30(4), 31-56.

Eckstein, Z. and Y. Weiss (2004). On the Wage Growth of Immigrants: Israel, 1990-2000. Journal of the European Economic Association 2(4), 665-695.

Felbermayr, G., W. Geis-Thöne, and W. K. Kohler (2010). Restrictive Immigration Policy in Germany: Pains and Gains foregone? Review of World Economics 146(1), 1-21.

Glorius, B. (2010). Go West: Internal Migration in Germany After Reunification. Revue belge de géographie (December 2012), 0-14.

Guerreiro, J., S. Rebelo, and P. Teles (2019, August). What is the Optimal Immigration Policy? Migration, Jobs and Welfare. (26154).

Heise, S. and T. Porzio (2019). Spatial Wage Gaps and Frictional Labor Markets. Working Paper.

Holter, H. A., D. Krueger, and S. Stepanchuk (2019). How Do Tax Progressivity and Household Heterogeneity Affect Laffer Curves? forthcoming: Quantitative Economics.

Iftikhar, Z. and A. Zaharieva (2019). General Equilibrium Effects of Immigration in Germany: Search and Matching Approach. Review of Economic Dynamics, 245-276. 
Imrohoroglu, S., S. Kitao, and T. Yamada (2017). Can Guest Workers Solve Japan's Fiscal Problems? Economic Inquiry.

Iskhakov, F., T. H. Jørgensen, J. Rust, and B. Schjerning (2017). The Endogenous Grid Method for Discrete-Continuous Dynamic Choice Models with (or without) Taste Shocks. Quantitative Economics 8, 317-365.

Katz, L. F. and K. M. Murphy (1992). Changes in Relative Wages, 1963-1987: Supply and Demand Factors. Quarterly Journal of Economics 107(1), 35-78.

Kirdar, M. G. (2012). Estimating the Impact of Immigrants on the Host Country Social Security System when Return Migration is an endogenous Choice. International Economic Review 53(2), 453-486.

Krueger, D. and A. Ludwig (2007). On the Consequences of Demographic Change for Rates of Returns to Capital, and the Distribution of Wealth and Welfare. Journal of Monetary Economics 54(1), 49 - 87.

Lagakos, D., B. Moll, N. Qian, and T. Schoellman (2018). Life-Cycle Human Capital Accumulation across Countries : Lessons from US Immigrants. Journal of Human Capital 12(2), 305-342.

Lee, R. D. and L. Carter (1992). Modeling and Forecasting U.S. Mortality. Journal of the American Statistical Association 87 (419), 659-671.

Lessem, R. and C. Sanders (2019). Immigrant Wage Assimilation in the United States: The Role of Occupational Upgrading. Working Paper.

Llull, J. (2018). Immigration, Wages, and Education: A Labour Market Equilibrium Structural Model. Review of Economic Studies.

Ludwig, A. and M. Reiter (2010). Sharing Demographic Risk - Who is Afraid of the Baby Bust? American Economic Journal: Economic Policy 2(4), 83-118.

McFadden, D. (1973). Conditional logit analysis of qualitative choice behavior.

Moreno-Galbis, E. and A. Tritah (2016). The Effects of Immigration in Frictional Labor Markets: Theory and Empirical Evidence from EU Countries. European Economic Review 84 (C), 76-98.

Nanos, P. and C. Schluter (2014). The Composition of Wage Differentials between Migrants and Natives. European Economic Review 65, 23 - 44.

Ottaviano, G. I. P. and G. Peri (2008, jul). Immigration and National Wages: Clarifying the Theory and the Empirics. NBER Working Papers 14188, National Bureau of Economic Research, Inc. 
Ottaviano, G. I. P. and G. Peri (2012). Rethinking the effect of immigration on wages. Volume 10, pp. 152-197. Oxford University Press.

Scharfbillig, M. and M. Weissler (2019). Heterogeneous Displacement Effects of Migrant Labor Supply - Experimental Evidence From Germany. SSRN Electronic Journal.

Stähler, N. (2017). A Model-Based Analysis of the Macroeconomic Impact of the Refugee Migration to Germany. Discussion Papers.

Storesletten, K. C. (2000). Sustaining Fiscal Policy Through Immigration. Journal of Political Economy 108, 300-323.

Weiss, Y., R. M. Sauer, and M. Gotlibovski (2003). Immigration, Search, and Loss of Skill. Journal of Labor Economics 21(3), 557-591. 


\section{Online Appendix}

\section{A Details and Proofs for the Simple Model}

\section{A.1 Definition of Equilibrium}

Definition 1. Given an initial capital stock $K_{0}$, an exogenous population $\left\{N_{t}(0, s, i), N_{t}(1, s, i)\right\}$ and government policy $\left\{\rho_{t}\right\}$ an equilibrium is a sequence of allocations and prices such that

1. Given wages $w_{t}(s, i)$, interest rates $R_{t+1}$ and policies $\tau_{t}, b_{t+1}(s, i)$ for each $t$ and each type $(s, i)$ the allocation $c_{t}(0, s, i), c_{t+1}(1, s, i), a_{t+1}(s, i)$ maximizes lifetime utility $(6)$ subject to the budget constraints (5).

2. Interest rates and wages $\left(R_{t}, w_{t}\right)$ satisfy the marginal product pricing equations (11) and (11), and type-specific wages are given by (13).

3. Government policies satisfy the budget constraint (10).

4. Markets clear:

(a) Labor Markets

$$
\begin{aligned}
L_{t}(h i) & =N_{t}(0, h i, n a) \\
L_{t}(l o, i) & =\epsilon(l o, i) N_{t}(0, l o, i) \quad \text { for } i \in\{n a, f o\}
\end{aligned}
$$

(b) Capital Market

$$
K_{t+1}=s_{t} w_{t} L_{t}
$$

(c) Goods Market

$$
C_{t}+K_{t+1}=K_{t}^{\alpha} L_{t}^{1-\alpha}
$$

Equilibrium in the small open economy is defined in a similar fashion, but the capital market clearing condition is replaced by the condition that the real interest rate $R_{t}=R$ is fixed by the world capital market, which then from the firm's optimality conditions pins down the constant wage $w_{t}=w(R)$ and capital-labor ratio $k_{t}=k(R)$. 


\section{A.2 Relative Wages as Functions of Demographics}

We summarize wages as functions of demographic variables as:

$$
\begin{aligned}
& \frac{L_{t}}{L_{t}(h i)}=\frac{\left(L_{t}(l o)^{1-\frac{1}{\sigma_{l h}}}+L_{t}(h i)^{1-\frac{1}{\sigma_{l h}}}\right)^{\frac{1}{1-\frac{1}{\sigma_{l h}}}}}{L_{t}(h i)}=\left(\left(\frac{L_{t}(l o)}{L_{t}(h i)}\right)^{1-\frac{1}{\sigma_{l h}}}+1\right)^{\frac{1}{1-\frac{1}{\sigma_{l h}}}} \\
& \frac{L_{t}}{L_{t}(l o)}=\left(\left(\frac{L_{t}(h i)}{L_{t}(l o)}\right)^{1-\frac{1}{\sigma_{l h}}}+1\right)^{\frac{1}{1-\frac{1}{\sigma l h}}} \\
& \frac{L_{t}(l o)}{L_{t}(h i)}=\frac{L_{t}\left(l_{o}\right)}{L_{t}(l o, f o)} \cdot \frac{L_{t}(l o, f o)}{L_{t}(h i)}=\left(\left(\frac{\epsilon(l o, n a)(1-\omega) \gamma_{t}^{n}}{\epsilon(l o, f o) \mu_{t}}\right)^{1-\frac{1}{\sigma_{n f}}}+1\right)^{\frac{1}{1-\frac{1}{\sigma_{n f}}}} \cdot \frac{\epsilon\left(l_{o}, f_{o}\right) \mu_{t}}{\omega \gamma_{t}^{n}} \\
& \frac{L_{t}(h i)}{L_{t}(l o)}=\frac{L_{t}(h i)}{L_{t}(l o, f o)} \cdot \frac{L_{t}(l o, f o)}{L_{t}(l o)}=\left(\left(\frac{\epsilon(l o, n a)(1-\omega) \gamma_{t}^{n}}{\epsilon(l o, f o) \mu_{t}}\right)^{1-\frac{1}{\sigma_{n f}}}+1\right)^{\frac{-1}{1-\frac{1}{\sigma_{n f}}}} \cdot \frac{\omega \gamma_{t}^{n}}{\epsilon(l o, f o) \mu_{t}} \\
& \frac{L_{t}(l o)}{L_{t}(l o, f o)}=\frac{\left(L_{t}(l o, n a)^{1-\frac{1}{\sigma_{n f}}}+L_{t}(l o, f o)^{1-\frac{1}{\sigma_{n f}}}\right)^{\frac{1}{1-\frac{1}{\sigma_{n f}}}}}{L_{t}(l o, n a)} \\
& =\left(\left(\frac{\epsilon(l o, n a)(1-\omega) \gamma_{t}^{n}}{\epsilon(l o, f o) \mu_{t}}\right)^{1-\frac{1}{\sigma_{n f}}}+1\right)^{\frac{1}{1-\frac{1}{\sigma_{n f}}}} \\
& \frac{L_{t}(l o)}{L_{t}(l o, n a)}=\frac{\left(L_{t}(l o, n a)^{1-\frac{1}{\sigma_{n f}}}+L_{t}\left(l o, f_{o}\right)^{1-\frac{1}{\sigma_{n f}}}\right)^{\frac{1}{1-\frac{1}{\sigma_{n f}}}}}{L_{t}(l o, n a)} \\
& =\left(\left(\frac{\epsilon(l o, f o) \mu_{t}}{\epsilon(l o, n a)(1-\omega) \gamma_{t}^{n}}\right)^{1-\frac{1}{\sigma_{n f}}}+1\right)^{\frac{1}{1-\frac{1}{\sigma_{n f}}}}
\end{aligned}
$$




$$
\begin{aligned}
& w_{t}(h i)=w_{t} \cdot\left(\frac{L_{t}}{L_{t}(h i)}\right)^{\frac{1}{\sigma_{l h}}}=w_{t} \cdot\left(\left(\frac{L_{t}(l o)}{L_{t}(h i)}\right)^{1-\frac{1}{\sigma_{l h}}}+1\right)^{\frac{\frac{1}{\sigma_{l h}}}{1-\frac{1}{\sigma_{l h}}}} \\
& =w_{t} \cdot\left(\left(\left(\left(\frac{\epsilon(l o, n a)(1-\omega) \gamma_{t}^{n}}{\epsilon(l o, f o) \mu_{t}}\right)^{1-\frac{1}{\sigma_{n f}}}+1\right)^{\frac{1}{1-\frac{1}{\sigma_{n f}}}} \cdot \frac{\epsilon(l o, f o) \mu_{t}}{\omega \gamma_{t}^{n}}\right)^{1-\frac{1}{\sigma_{l h}}}+1\right)^{\frac{1}{\sigma_{l h}-1}} \\
& =w_{t} \cdot\left(\left(\left(\left(\frac{\epsilon(l o, n a)(1-\omega)}{\omega}\right)^{1-\frac{1}{\sigma_{n f}}}+\left(\frac{\epsilon(l o, f o) \mu_{t}}{\omega \gamma_{t}^{n}}\right)^{1-\frac{1}{\sigma_{n f}}}\right)^{\frac{1}{1-\frac{1}{\sigma_{n f}}}} \cdot\right)^{1-\frac{1}{\sigma_{l h}}}+1\right)^{\frac{1}{\sigma_{l h}-1}} \\
& =w_{t} \cdot \mathcal{W}_{h i}\left(\mu_{t} / \gamma_{t}^{n}\right) \\
& w_{t}(l o, n a)=w_{t} \cdot \epsilon(l o, n a) \cdot\left(\frac{L_{t}}{L_{t}(l o)}\right)^{\frac{1}{\sigma_{l h}}} \cdot\left(\frac{L_{t}(l o)}{L_{t}(l o, n a)}\right)^{\frac{1}{\sigma_{n f}}} \\
& =w_{t} \cdot \epsilon(l o, n a) \\
& \left.\cdot\left(\left(\left(\frac{\epsilon(l o, n a)(1-\omega)}{\omega}\right)^{1-\frac{1}{\sigma_{n f}}}+\left(\frac{\epsilon\left(l o, f_{O}\right) \mu_{t}}{\omega \gamma_{t}^{n}}\right)^{1-\frac{1}{\sigma_{n f}}}\right)^{\frac{-1}{1-\frac{1}{\sigma_{n f}}}}\right)^{1-\frac{1}{\sigma_{l h}}}+1\right)^{\frac{1}{\sigma_{l h}-1}} \\
& \cdot\left(\left(\frac{\epsilon(l o, f o) \mu_{t}}{\epsilon(l o, n a)(1-\omega) \gamma_{t}^{n}}\right)^{1-\frac{1}{\sigma_{n f}}}+1\right)^{\frac{1}{\sigma_{n f}-1}} \\
& =w_{t} \mathcal{W}_{l o}\left(\mu_{t} / \gamma_{t}^{n}\right) \cdot \mathcal{W}_{n a}\left(\mu_{t} / \gamma_{t}^{n}\right)
\end{aligned}
$$

It follows from direct inspection that $\mathcal{W}_{h i}\left(\mu_{t} / \gamma_{t}^{n}\right), \mathcal{W}_{n a}\left(\mu_{t} / \gamma_{t}^{n}\right)$ are strictly increasing in $\mu_{t} / \gamma_{t}^{n}$ and $\mathcal{W}_{l o}\left(\mu_{t} / \gamma_{t}^{n}\right)$ is strictly decreasing in $\mu_{t} / \gamma_{t}^{n}$.

\section{A.3 Proof of Lemma 1 and Theorem 1}

For lemma 1, we want to arrive at an expression for $\gamma_{t+1}^{L}=\frac{L_{t+1}}{L_{t}}$. Recall from (3) and (4) that

$$
\begin{aligned}
L_{t} & =\left(L_{t}(l o)^{1-\frac{1}{\sigma_{l h}}}+L_{t}(h i)^{1-\frac{1}{\sigma_{l h}}}\right)^{\frac{1}{1-\frac{1}{\sigma_{l h}}}} \\
L_{t}(l o) & =\left(L_{t}(l o, n a)^{1-\frac{1}{\sigma_{n f}}}+L_{t}(l o, f o)^{1-\frac{1}{\sigma_{n f}}}\right)^{\frac{1}{1-\frac{1}{\sigma_{n f}}}}
\end{aligned}
$$


Work on (4):

$$
\begin{aligned}
L_{t}(l o) & =\left(\left(\epsilon(l o, n a) N_{t}(0, l o, n a)\right)^{1-\frac{1}{\sigma_{n f}}}+\left(\epsilon(l o, f o) N_{t}(0, l o, f o)\right)^{1-\frac{1}{\sigma_{n f}}}\right)^{\frac{1}{1-\frac{1}{\sigma_{n f}}}} \\
& =\left(\left(\epsilon(l o, n a)(1-\omega) \gamma_{t}^{n} N_{t-1}(0)\right)^{1-\frac{1}{\sigma_{n f}}}+\left(\epsilon(l o, f o) \mu_{t} \gamma_{t}^{n} N_{t-1}(0)\right)^{1-\frac{1}{\sigma_{n f}}}\right)^{\frac{1}{1-\frac{1}{\sigma_{n f}}}} \\
& =\left(\left(\epsilon(l o, n a)(1-\omega) \gamma_{t}^{n}\right)^{1-\frac{1}{\sigma_{n f}}}+\left(\epsilon(l o, f o) \mu_{t} \gamma_{t}^{n}\right)^{1-\frac{1}{\sigma_{n f}}}\right)^{\frac{1}{1-\frac{1}{\sigma_{n f}}}} N_{t-1}(0) \\
& =\Lambda\left(\gamma_{t}^{n}, \mu_{t}\right) N_{t-1}(0)=\Lambda_{t} N_{t-1}(0)
\end{aligned}
$$

Use this in (3) to get

$$
\begin{aligned}
L_{t} & =\left(\left(\Lambda(\cdot) N_{t-1}(0)\right)^{1-\frac{1}{\sigma_{l h}}}+\left(\omega \gamma_{t}^{n} N_{t-1}(0)\right)^{1-\frac{1}{\sigma_{l h}}}\right)^{\frac{1}{1-\frac{1}{\sigma_{l h}}}} \\
& =\left((\Lambda(\cdot))^{1-\frac{1}{\sigma_{l h}}}+\left(\omega \gamma_{t}^{n}\right)^{1-\frac{1}{\sigma_{l h}}}\right)^{\frac{1}{1-\frac{1}{\sigma_{l h}}}} N_{t-1}(0) \\
& =\Omega\left(\Lambda\left(\gamma_{t}^{n}, \mu_{t}\right), \gamma_{t}^{n}\right) N_{t-1}(0)=\Omega_{t}\left(\Lambda_{t}, \gamma_{t}^{n}\right) N_{t-1}(0) .
\end{aligned}
$$

Thus we get

$$
\begin{aligned}
\gamma_{t+1}^{L} & =\frac{\Omega\left(\Lambda\left(\gamma_{t+1}^{n}, \mu_{t+1}\right), \gamma_{t+1}^{n}\right) N_{t}(0)}{\Omega\left(\Lambda\left(\gamma_{t}^{n}, \mu_{t}\right), \gamma_{t}^{n}\right) N_{t-1}(0)} \\
& =\frac{\Omega\left(\Lambda\left(\gamma_{t+1}^{n}, \mu_{t+1}\right), \gamma_{t+1}^{n}\right)}{\Omega\left(\Lambda\left(\gamma_{t}^{n}, \mu_{t}\right), \gamma_{t}^{n}\right)} \gamma_{t} \\
& =\frac{\Omega\left(\Lambda\left(\gamma_{t+1}^{n}, \mu_{t+1}\right), \gamma_{t+1}^{n}\right)}{\Omega\left(\Lambda\left(\gamma_{t}^{n}, \mu_{t}\right), \gamma_{t}^{n}\right)}\left(\gamma_{t}^{n}+\mu_{t}\right) \\
& =\frac{\Omega_{t+1}}{\Omega_{t}}\left(\gamma_{t}^{n}+\mu_{t}\right) \\
& =\gamma_{t}^{n}+\mu_{t} \text { if } \gamma_{t+1}^{n}=\gamma_{t}^{n}, \text { and } \mu_{t+1}=\mu_{t}
\end{aligned}
$$

We make the following:

Observation 1. 1. Fix $\gamma^{n}$ and consider a permanent change of $\mu_{t}$ from $\mu^{l}>0$ to $\mu^{h}>\mu^{l}$ in period $t$. Since $\Lambda_{t+1}=\Lambda_{t}$ we have $\Omega_{t+1}=\Omega_{t}$ and thus $\gamma_{t+1}^{L}$ jumps to $\gamma^{n}+\mu^{l}$.

2. Fix $\mu$ and consider a permanent change of $\gamma^{n}$ from $\gamma^{n l}>0$ to $\gamma^{n h}>\gamma^{n l}$ in period $t$. Since $\Lambda_{t+1}=\Lambda_{t}$ and $\Omega_{t+1}\left(\Lambda_{t+1}, \gamma^{n h}\right)=\Omega_{t}\left(\Lambda_{t}, \gamma^{n h}\right)$ we have that $\gamma_{t+1}^{L}$ jumps to $\gamma^{n h}+\mu$. 
The proof of theorem 1 then follows directly from lemma 1 as well as propositions 2 and 3. The only non-trivial part is to sign the general equilibrium effect. For this note that

$$
\begin{aligned}
(1+\beta) \ln \left(w_{t}\right)+\beta \ln \left(R_{t+1}\right) & =(1+\beta) \ln \left((1-\alpha) k_{t}^{\alpha}\right)+\beta \ln \left(\alpha k_{t+1}^{\alpha-1}\right) \\
& =v+(1+\beta) \alpha \ln \left(k_{t}\right)-(1-\alpha) \beta \ln \left(k_{t+1}\right) \\
& =v+(1+\beta) \alpha \ln \left(k_{t}\right)-(1-\alpha) \beta\left[\ln \left(s_{t}\right)+\alpha \ln \left(k_{t}\right)-\ln \left(\gamma_{t+1}^{L}\right)\right] \\
& =v+\alpha(1+\alpha \beta) \ln \left(k_{t}\right)-(1-\alpha) \beta\left[\ln \left(s_{t}\right)-\ln \left(\gamma_{t+1}^{L}\right)\right] \\
& =v+\alpha(1+\alpha \beta) \ln \left(K_{t}\right)-(1-\alpha) \beta \ln \left(s_{t}\right) \\
& -[\alpha(1+\alpha \beta)+(1-\alpha) \beta] \ln \left(L_{t}\right)+(1-\alpha) \beta \ln \left(L_{t+1}\right) .
\end{aligned}
$$

where $v$ is a constant. The period $t$ capital stock $K_{t}$ is pre-determined. The saving rate $s_{t}$ increases with the per-capita immigration cost $\kappa_{t+1}$ which in turn rises as more migrants come in, increasing the capital-labor ratio in period $t+1$ and thus reducing the real interest rate. This is the first negative general equilibrium effect (which would be absent if there are no resource costs for the newley arriving migrants, i.e. if $\kappa_{t+1}=0$ ). Second, both $L_{t}$ as well as $\gamma_{t+1}^{L}=L_{t+1} / L_{t}$ increase when $\mu_{t}$ increases permanently. As long as $\alpha$ is sufficiently large (trivially, if $\alpha=1$ ), or as long as $\frac{\partial \ln \left(L_{t}\right)}{\partial \mu} \approx \frac{\partial \ln \left(L_{t+1}\right)}{\partial \mu}$ (both of these terms only depend on model-exogenous variables) the general equilibrium effect of a permanent increase in migration flows is negative.

Remark 1. Also note that in the absence of a resource cost $\left(\kappa_{t+1}=0\right)$ the saving rate is invariant to demographics, and an increase in migration triggers a decline in the current capital-labor ratio $k_{t}$ and a further decline in future capita-labor ratios $k_{t+s}$ through the permanent increase in the growth rate of labor $\gamma_{t+s}^{L}$. In the long-run the GE effect of these declines is negative as long as the economy remains dynamically efficient. To see this, observe that for all $t \geq 1$ the welfare difference along the transition is

$$
\begin{aligned}
\Delta\left[(1+\beta) \ln \left(w_{t}\right)+\beta \ln \left(R_{t+1}\right)\right] & =(1+\beta)\left(\ln \left(w_{t}\right)-w_{0}\right)+\beta\left(\ln \left(R_{t+1}\right)-R_{0}\right) \\
& =(1+\beta) \alpha\left(\ln \left(k_{t}\right)-\ln \left(k_{0}\right)\right)-(1-\alpha) \beta\left(\ln \left(k_{t+1}\right)-\ln \left(k_{0}\right)\right) .
\end{aligned}
$$

For $t \rightarrow \infty$ this term is negative if

$$
\frac{\alpha}{1-\alpha}>\frac{\beta}{1+\beta}
$$

It is straightforward to verify from the corresponding social planner's problem that this is the condition for dynamic efficiency of the economy. In the short run, for the period $t=1$ old generation the effect is positive because the wage effect is absent. For all newborn generations 
along the transition, the effect is negative if the capital share $\alpha$ is sufficiently large-notice that dynamic efficiency is thus only a necessary condition for the effect to be negative for all newborns along the transition-, because for all $t \geq 1$ the welfare change is negative if

$$
\begin{aligned}
& (1+\beta) \alpha\left|\left(\ln \left(k_{t}\right)-\ln \left(k_{0}\right)\right)\right|-(1-\alpha) \beta\left|\left(\ln \left(k_{t+1}\right)-\ln \left(k_{0}\right)\right)\right|>0 \\
\Leftrightarrow \quad & \frac{\alpha}{1-\alpha} \frac{\left|\left(\ln \left(k_{t}\right)-\ln \left(k_{0}\right)\right)\right|}{\left|\left(\ln \left(k_{t+1}\right)-\ln \left(k_{0}\right)\right)\right|}>\frac{\beta}{1+\beta} .
\end{aligned}
$$

and by the monotonic decline of the capital stock we know that $\frac{\left|\left(\ln \left(k_{t}\right)-\ln \left(k_{0}\right)\right)\right|}{\left|\left(\ln \left(k_{t+1}\right)-\ln \left(k_{0}\right)\right)\right|}<1$.

\section{B Quantitative Model Appendix}

\section{B.1 Assimilation Flows}

We construct net migration numbers at the net addition to the population stock from migration flows in the next period, $M_{t+1}(j+1, i, g)$, from which we then compute the migration rates $\mu_{t}(j, a s, g)=\frac{M_{t+1}(j+1, i, g)}{N_{t}(j, i, g)}$. Denoting by $M_{t+1}^{f}(j+1, a s, g)$ the inflow from foreign countries to the group of asylum seekers, the net immigration flow to group as is

$$
M_{t+1}(j+1, a s, g)=M_{t+1}^{f}(j+1, a s, g)-\left(\pi^{l}+\left(1-\pi^{l}\right) \pi^{a r}\right) N_{t}(j, a s, g) \psi_{t}(j, a s, g)
$$

and therefore

$$
\mu_{t}(j, a s, g)=\mu_{t}^{f}(j, a s, g)-\left(\pi^{l}+\left(1-\pi^{l}\right) \pi^{a r}\right) \psi_{t}(j, a s, g) .
$$

Denoting by $M_{t+1}^{f}(j+1, r w, g)$ the inflow from foreign countries to population group $r w$, the net inflow to the population group $r w$ is

$$
\begin{array}{r}
M_{t+1}(j+1, r w, g)=M_{t+1}^{f}(j+1, r w, g)+\left(1-\pi^{l}\right) \pi^{a r} \psi_{t}(j, a s, g) N_{t}(j, a s, g) \\
-\pi^{r h} \psi_{t}(j, r w, g) N_{t}(j, r w, g)
\end{array}
$$

and thus

$$
\mu_{t}(j, r w, g)=\mu_{t}^{f}(j, r w, g)+\left(1-\pi^{l}\right) \pi^{a r} \psi_{t}(j, a s, g) \frac{N_{t}(j, a s, g)}{N_{t}(j, r w, g)}-\pi^{r h} \psi_{t}(j, r w, g)
$$


Correspondingly, denoting by $M_{t+1}^{f}(j+1, h o, g)$ the inflow from foreign countries to population group ho, the total inflow to population group ho is

$$
M_{t+1}(j+1, h o, g)=M_{t+1}^{f}(j+1, h o, g)+\pi^{r h} \psi_{t}(j, r w, g) N_{t}(j, r w, g)
$$

and thus

$$
\mu_{t}(j, h o, g)=\mu_{t}^{f}(j, h o, g)+\pi^{r h} \psi_{t}(j, r w, g) \frac{N_{t}(j, r w, g)}{N_{t}(j, h o, g)}
$$

\section{B.2 First-Order Conditions of Firm Problem}

Denote by $k_{t}=\frac{K_{t}}{A_{t} L_{t}}$ the "capital intensity", respectively the capital stock per efficiency unit of labor. Then, the first-order conditions of the static firm problem are given by

$$
\begin{aligned}
r_{t} & =\alpha k_{t}^{-\frac{1}{\vartheta}}\left(\alpha k_{t}^{1-\frac{1}{\vartheta}}+(1-\alpha)\right)^{\frac{\frac{1}{\vartheta}}{1-\frac{1}{\vartheta}}}-\delta \\
w_{t} & =A_{t}(1-\alpha)\left(\alpha k_{t}^{1-\frac{1}{\vartheta}}+(1-\alpha)\right)^{\frac{\frac{1}{\vartheta}}{1-\frac{1}{\vartheta}}} \\
w_{t}(s) & =w_{t}\left(\frac{L_{t}}{L_{t}(s)}\right)^{\frac{1}{\sigma_{l m h}}} \\
w_{t}(\bar{j}, s) & =w_{t}(s) \\
w_{t}(\bar{j}, s, n a) & =w_{t}(\bar{j}, s)\left(\frac{L_{t}(\bar{j}, s)}{L_{t}(\bar{j}, s, n a)}\right)^{\frac{1}{\sigma_{n f}}}, \\
\tilde{w}_{t}(\bar{j}, s, f o) & =w_{t}(\bar{j}, s)\left(\frac{L_{t}(\bar{j}, s)}{\tilde{L}_{t}(\bar{j}, s, f o)}\right)^{\frac{1}{\sigma_{n f}}}, \\
w_{t}(\bar{j}, s, h o) & =\tilde{w}_{t}(\bar{j}, s, f o)\left(\frac{\tilde{L}_{t}(\bar{j}, s, f o)}{L_{t}(\bar{j}, s, h o)}\right)^{\frac{1}{\sigma_{h r}}} \\
w_{t}(\bar{j}, s, o) & =\tilde{w}_{t}(\bar{j}, s, f o)\left(\frac{\tilde{L}_{t}(\bar{j}, s, f o)}{\sum_{o \in\{r w, a s\}} L_{t}(s, o)}\right)^{\frac{1}{\sigma_{h r}}} \quad \text { for } o \in\{r w, a s\} .
\end{aligned}
$$

We then get the age $j$, skill $s$, nationality $i$-specific aggregate wage component $w_{t}(j, s, i)=$ $w_{t}(\bar{j}, s, i)$ if $j \in\left[j_{l}(\bar{j}), \ldots, j_{h}(\bar{j})\right]$.

\section{B.3 Annuity Income Stream of Leavers}

Total wealth of a leaver includes the value of assets at the end of period $t$ at age $j$ net of fraction $\pi^{c}$ of confiscated assets by the government of the country the leaver remigrates to, 
a one time lump-sum payment by the German government $b_{t}^{l}$, and the discounted value of future labor income. We assume that in the country a household remigrates to it works full-time, $l=l_{n}$, does not pay or receive any transfers from a social insurance institution, and retires exogenously at age $j_{r}$. Accidental bequests are taxed at a confiscatory rate. We compute the continuation value in a partial equilibrium, taking the current period wage $w_{t}$ and the interest rate in the period of leaving $r_{t}$ as given.

Denote by $a_{t}^{\prime}$ the savings of a leaver during the leaving period, i.e, in the last period in Germany. Initial assets after confiscation at the beginning of period $t+1$ in the country the leaver migrates to are $a_{t+1}=\left(1-\pi^{c}\right) a_{t}^{\prime}$. Total wealth of a leaver with education $s$ and gender $g$ in period $t+1$, age $j+1$, is accordingly given by

$$
W_{t+1}(j+1, s, g)=a_{t+1}\left(1+r_{t}\right)+b_{t}^{l}+\eta \cdot \sum_{p=j+1}^{j_{r}-1}\left(\frac{1}{1+r_{t}}\right)^{p-(j+1)} \epsilon(p, s, i, g) w_{t}(p, s, g) l_{n}
$$

where $\eta \in(0,1)$ is a productivity scaling parameter, reflecting lower productivity in the respective country as well as linear labor income taxes. The according annuity stream is

$$
y_{t+1}^{a}(s, g)=\frac{r_{t}}{1+r_{t}} \frac{\left(1+r_{t}\right)^{J-j}}{\left(1+r_{t}\right)^{J-j}-1} W_{t+1}(j+1, s, g)
$$

\section{B.4 Gains and Loss Term}

For consumption equivalent variation of a cohort born in period $t-j$ for period $t$ state variables age $j$, education $s$, nationality $i$, gender $g$, asset holdings $a$, denoted by $g_{t-j}^{c}(j, s, i, g, a)$ and corresponding cross-sectional $\Phi_{t}(j, s, i, g, a)$ in the baseline demographic scenario, we compute the average consumption equivalent variation

$$
g_{t-j}^{c}(j, s, i, g)=\int g_{t-j}^{c}(j, s, i, g, a) \Phi_{t}(j, s, i, g, d a) .
$$

For period 2013 we compute the above CEV for all cohorts $t-j$, ages $j=0, \ldots, J$, and consider the actual asset position and employment state in period 2013. For cohorts born after 2013 we evaluate the $\mathrm{CEV}$ at $j=0, a=0$, only. The period $t$ consumption of the respective group given the consumption policy function in the baseline demographic scenario $c_{t}(j, s, i, g, a)$ in turn is

$$
c_{t}(j, s, i, g)=\int c_{t}(j, s, i, g, a) \Phi_{t}(j, s, i, g, d a)
$$

These objects form the basis of the computation of the net gain term in equation (47). 


\section{B.5 Recursive Household Problem}

State Variables. We collect state variables as follows, also see Table 1: age $j \in\left\{j_{a}, \ldots, J\right\}$, education $s \in\{l o, m e, h i\}$, economic nationality $i \in\{n a, h o, r w, a s\}$, gender $g \in\{f e, m a\}$, employment status $e \in\{e m, r e\}$, and assets $a \in \mathcal{A}$.

For asylum seekers the problem is slightly more complex because of the leaving shock and the assimilation shock. Also, immigrants from the rest of the world face an assimilation shock. We therefore first describe the problems of groups $i \in\{n a, h o\}$ and then turn to relevant extensions for the remaining two population groups.

Dynamic Problem of Retired Households, $j \in\left\{j_{r}, \ldots, J\right\}, i \in\{n a, h o\}, e=r e$. Retired agents solve ${ }^{37}$

$$
V_{t}(j, s, i, g, e, a)=\max _{c, a^{\prime}}\left\{U\left(\frac{c}{1+\zeta n}, 1\right)+\beta \psi_{t}(j, i, g) V_{t+1}\left(j+1, s, i, g, e, a^{\prime}\right)\right\}
$$

subject to

$$
\begin{aligned}
& a^{\prime}=\left(a+t r_{t}\right)\left(1+r_{t}\left(1-\tau_{t}^{k}\right)\right)+y_{t}^{p}-\left(c\left(1+\tau_{t}^{c}\right)+T_{t}\left(y_{t}^{p}\right)\right) \geq 0 \\
& y_{t}^{p}=\left(1-\tau_{t}^{h}\right) b_{t}^{p}(s, i, g) .
\end{aligned}
$$

Dynamic Problem of Working Households in Last Working Period, $j=j_{r}-1, i \in$ $\{n a, h o\}, e=e m$. In the last period of work, $j_{r}-1$, the value function is the expected value of the maximized value functions of the discrete choice specific value functions $J\left(\cdot, l_{i}\right)$ from working $l_{i} \in\left\{l_{1}, \ldots, l_{n}\right\}$ hours, which is, given the Gumbel distributed taste shocks $\varepsilon$ with scale parameter $\varsigma$,

$$
V_{t}(j, s, i, g, e=e m, a)=\varsigma \log \left[\sum_{k=1}^{n} \exp \left\{\frac{J_{t}\left(\cdot, l_{k}\right)}{\varsigma}\right\}\right]
$$

with according choice probabilities for alternative $k$

$$
\pi_{t}\left(j, s, i, g, e=e m, a, l=l_{k}\right)=\frac{\exp \left(\frac{J_{t}\left(\cdot, l=l_{k}\right)}{\varsigma}\right)}{\sum_{m=1}^{n} \exp \left(\frac{J_{t}\left(\cdot, l=l_{m}\right)}{\varsigma}\right)} .
$$

\footnotetext{
${ }^{37}$ Recall that $\psi_{t} J, i, g=0$ so that terminal (and trivial) decision problem of singles and couples at age $J$ are nested in this description.
} 
where $J_{t}\left(\cdot, l_{k}\right)$ is the choice specific value function for working $l_{k} \in\left\{l_{1}, \ldots, l_{n}\right\}$ hours

$$
\begin{aligned}
J_{t}\left(j, s, i, g, e=e m, a, l=l_{k}\right) & = \\
& \max _{c, a^{\prime}}\left\{U\left(c, 1-l_{k}\right)+\beta \psi_{t}(j, i, g) V_{t+1}\left(j+1, s, i, g, e=r e, a^{\prime}\right)\right\}
\end{aligned}
$$

subject to

$$
\begin{aligned}
& a^{\prime}=\left(a+t r_{t}\right)\left(1+r_{t}\left(1-\tau_{t}^{k}\right)\right)+y_{t}-c\left(1+\tau_{t}^{c}\right)-T_{t}\left(y_{t}\right) \geq 0 \\
& y_{t}=\left(1-\tau_{t}^{p}-\tau_{t}^{h}\right) w_{t}(j, s, i) \epsilon(j, s, i, g) l_{k}
\end{aligned}
$$

Dynamic Problem of Working Households in Working Period $j \in\left\{j_{s}, \ldots, j_{r}-2\right\}, i \in$ $\{n, h, r\}, e=e m$. The structure is the same as previously, where continuation values at $t, j$ are the value functions $V_{t+1}\left(j+1, s, i, g, e=e m, a^{\prime}\right)$.

Dynamic Problem of Households $i \in\{n a, h o\}, j \in\left\{j_{a}, \ldots, j_{s}\right\}, e=e m$. The dynamic problem is the same as described above, but the current period labor productivity is further shifted by factor $\varrho(s) \in(0,1)$.

Modifications for Asylees, $i=a s$. Due to differences in access to the social insurance system and transfer payments to asylees as well as labor market restrictions, the problem of asylees in the first year of entry is different from other years, which we store in indicator $\mathbb{1}_{a}$. At the end of each period conditional on surviving asylum seekers face the risk of having to leave with respective probability $\pi^{l}$ and, conditional on not leaving, they may assimilate to population group $r w$ with probability $\pi^{a r}$, thus the unconditional probability of assimilating to group $r w$ is $\left(1-\pi^{l}\right) \pi^{a r}$ and the unconditional probability of staying in population group as is $\left(1-\pi^{l}\right)\left(1-\pi^{a r}\right)$. For the remainder of the description we focus on asylum seekers during the working period and spell out later the adjustments needed for other stages of the life-cycle.

First, we compute the continuation value in case of leaving. An asylee being forced to leave at age $j+1$ receives in each period a permanent income stream $y^{a}(s, g)$, which we compute for both partners in a couple according to equation (54). The household enjoys flow utility from consumption of the annuity in each period and is assumed to work fulltime, $U\left(y^{a}(s, g), 1-l_{n}\right)$, and thus the value function in case of being forced to leave can be 
computed recursively as

$$
\begin{aligned}
& V^{l}\left(j+1, s, i=a s, a^{\prime}\right)= \\
& U\left(\frac{y^{a}(s, g)}{1+\zeta n+\xi}, 1-l_{n}\right)+\beta \psi_{t+1}(j+1, a s, g) V^{l}\left(j+2, s, i=a s, a^{\prime \prime}\right)
\end{aligned}
$$

subject to

$$
a^{\prime \prime}=a^{\prime}(1+r)+\mathbb{1}_{j \leq j_{r}-1} \cdot \eta \cdot w_{t}(j+1, s, a s, g) l_{n}-y^{a}(s, g)
$$

where indicator $\mathbb{1}_{j \leq j_{r}-1}$ is equal to one if the household is of working age $j \leq j_{r}-1, r$ is the return on assets in the period of leaving the country, and the initial asset position is $\left(1-\pi^{c}\right) \bar{a}_{t}^{\prime}$, where $\bar{a}_{t}^{\prime}$ are savings during the leaving period.

Problem of Asylum Seekers at Age $j \in\left\{j_{s}, \ldots, j_{r}-2\right\}$. The problem of an asylum seeker in the working period is

$$
\begin{array}{r}
V_{t}(j, s, a s, e=e m, a)=\max _{c, a^{\prime}}\left\{U\left(\frac{c}{1+\zeta n+\xi}, 1-\mathbb{1}_{a} \underline{l}^{a}-\left(1-\mathbb{1}_{a}\right) \bar{l}^{a}\right)\right. \\
\beta \psi_{t}(j, i, g)\left(\pi^{l} V_{t+1}^{l}\left(j+1, s, a s, a^{\prime}\right)+\left(1-\pi^{l}\right)\left(\pi^{a r} V_{t+1}\left(j+1, s, i=r w, e^{\prime}=e m, a^{\prime}\right)\right.\right. \\
\left.\left.\left.+\left(1-\pi^{a r}\right) V_{t+1}\left(j+1, s, i=a s, e^{\prime}=e m, a^{\prime}\right)\right)\right)\right\}
\end{array}
$$

subject to

$$
\begin{aligned}
& a^{\prime}=\left(a+t r_{t}\right)\left(1+r_{t}\left(1-\tau_{t}^{k}\right)\right)+y_{t}+b_{t}^{a}(n)-\left(c\left(1+\tau_{t}^{c}\right)+T_{t}\left(y_{t}\right)\right) \geq 0 \\
& y_{t}=w_{t}(s, j, a s, g) \epsilon(s, j, a s, g)\left(\mathbb{1}_{a} \underline{l}^{a}+\left(1-\mathbb{1}_{a}\right) \bar{l}^{a}\right) .
\end{aligned}
$$

Immigrants from Other Population Groups. Unlike asylum seekers groups rw, ho have full access to the labor market and to the German social insurance system in the first period after arrival. Immigrants from group $r w$ face in each period the probability $\pi^{r h}$ to assimilate to group ho, which they take into account in their continuation values.

\section{Calibration Appendix}

Table 5 summarizes the exogenously calibrated and Table 6 the endogenously calibrated parameters of the model. 
Table 5: Exogenous Calibration Parameters

\begin{tabular}{|c|c|c|}
\hline Parameter & Interpretation & Value \\
\hline \multicolumn{3}{|c|}{ Population } \\
\hline$N_{t}(j, i)$ & Population Stock Data & Section 6.2 \\
\hline$j_{a}$ & Age at labor market entrance & 17 \\
\hline$j_{l}$ & Age of education completion low-skilled & 16 \\
\hline$j_{m}$ & Age of education completion middle-skilled & 20 \\
\hline$j_{h}$ & Age of education completion high-skilled & 24 \\
\hline$j_{f}$ & Fertility Age & 15 \\
\hline$j_{c}$ & Age of completing fertility & 50 \\
\hline$j_{r}$ & Statutory Retirement Age & 66 \\
\hline$J$ & Max. Lifetime & 100 \\
\hline$\left\{\psi_{t}(j, i, g)\right\}$ & Survival rates & Section 6.2 \\
\hline$\phi$ & Fraction of baby girls & 0.48 \\
\hline$\left\{\chi_{t}(j, i)\right\}$ & Fertility rates & Section 6.2 \\
\hline$\pi^{l}$ & Leaving probability & 0.06 \\
\hline$\pi^{a r}$ & Assimilation probability $a s \Rightarrow r w$ & 0.008 \\
\hline$\pi^{r h}$ & Assimilation probability $r w \Rightarrow h i$ & 0.060 \\
\hline$\phi(s, i)$ & Fraction of skill $s$ among population $i$ & Table 3 \\
\hline \multicolumn{3}{|c|}{ Endowments } \\
\hline$\epsilon(j, s, i)$ & Age Profile & Figure 16 \\
\hline$\epsilon(g)$ & Productivity Shifter by Gender & {$[0.8074,1]$} \\
\hline$\left\{l_{1}, \ldots, l_{n}\right\}$ & Discrete labor supply levels & $\{0.036,0.18,0.36\}$ \\
\hline$\left\{\underline{l}^{a}, \bar{l}^{a}\right\}$ & Fraction of full-time work of group as & $\{0.109,0.369\}$ \\
\hline$\eta$ & Relative productivity of leavers & 0.45 \\
\hline$\varrho(s)$ & Productivity loss in education & 0.5 \\
\hline$\pi^{c}$ & Confiscation rate of assets for leaving asylum seekers & 1 \\
\hline \multicolumn{3}{|c|}{ Preferences } \\
\hline$\theta$ & Relative risk aversion parameter & 1 \\
\hline$\sigma_{\epsilon}$ & Scale parameter of taste shocks & 0.1 \\
\hline
\end{tabular}




\begin{tabular}{llc}
\hline \multicolumn{2}{c}{ Production } \\
\hline$\alpha$ & Capital share & 0.33 \\
$\delta$ & Depreciation rate & 0.05 \\
$\lambda$ & Rate of technological progress & 0.015 \\
$\vartheta$ & Elast. of substitution b/w capital and labor & 1 \\
$\sigma_{l m h}$ & Elast. of substitution b/w labor of different skill levels & 3.05 \\
$\sigma_{n f}$ & Elast. of substitution b/w fo and na & 13.22 \\
$\sigma_{h r}$ & Elast. of substitution b/w ho and $r w$ & 22.61 \\
\hline & \multicolumn{1}{c}{ Government } \\
\hline$\alpha^{p}$ & Sensitivity parameter in the pension formula & $0(0.25)$ \\
$\tau^{p}$ & Pension contribution rate & Figure 17 \\
$\tau^{h}$ & Health system contribution rate & Figure 17 \\
$\iota$ & Private contribution factor & $0(0.04)$ \\
$b^{a}$ & Transfer payments to asylum seekers & Section 6.6 .1 \\
$\left\{b^{h}(j)\right\}$ & Health insurance payments & Figure 18 \\
$\tau^{c}$ & Consumption tax rate (in steady-state) & $19 \%$ \\
$\tau^{k}$ & Capital income tax rate & $25 \%$ \\
$\left\{G / Y_{t}\right\}$ & Government consumption to GDP ratio & Section 6.6 .2 \\
\hline \hline
\end{tabular}

Notes: Exogenous calibration parameters from various source described in Section 6.

Table 6: Endogenous Calibration Parameters

\begin{tabular}{lcccc}
\hline \hline Parameter & Interpretation & Value & Target & Target Value \\
\hline$\beta$ & Discount Factor & 0.9827 & $K / Y$ & 3.66 \\
$\phi(g)$ & Util. Wgths. & {$[2.846,1.227]$} & Uncond. Hours/Full-Time Hours & {$[0.475,0.815]$} \\
$\omega_{0}$ & Level Param. Tax Func. & 0.8416 & $G / Y$ & $19.12 \%$ \\
\hline \hline
\end{tabular}

Notes: Parameters calibrated endogenously by matching 2010 auxiliary steady state moments. 


\section{C.1 Population Model}

Population data is from the German Federal Statistical Office (Statistisches Bundesamt /Destatis; HMD) and from the Central Foreign Population Registry (Ausländerzentralstatistik, AZR). In this data foreigners include all persons who do not have German citizenship, and we have explicit information on the stock of first and second generation foreigners. A first generation foreigner is a person that was born outside Germany, whereas a second generation foreigner in the data is born in Germany but holds foreign nationality. By our economic perspective we consider second generation foreigners as natives irrespective of their legal citizenship, cf. equation (30), and accordingly assign them to population group with "nationality" na. With this assumption, we first construct the age-specific population stock $N_{t}(j, i, g)$ for groups $i \in\{n a, h o, r w, a s\}$ for the years $2008-2019$.

Next, we impute from this data the implied net addition to the population stock $M_{t}(j, i, g)$ from the law of motion of the population in equation (29), taking into account the adjustments of the dynamics that are implied by the assimilation probability $\pi^{a r}$ and the leave probability $\pi^{l}$. We refer to the net change of the stock also as the migration flow. ${ }^{38}$

To compute this net flow from (29) we also need data on age, group, and time specific mortality rates. We take those from the Human Mortality Database (HMD) for years 19602017 and, since we lack data on group specific mortality rates, we assume that all immigrants immediately after entry have the same mortality process as the average German population and thus set $\psi_{t}(j, i, g)=\psi_{t}(j, g) \forall i$.

For the predictions of the population beyond 2019 we make the following assumptions:

1. For all groups $\{n a, r w, h o, a s\}$ we compute the average age distribution of constructed net migration numbers $\bar{M}(j, i, g)$ over the years 2007-2018. We assume that aggregate migration in each group reverts to a long-run average until 2022. This reversal takes place according to the timing assumptions for each scenario described in Section 6.1. To compute long-run average migration in each group we assume - consistent with conventional assumptions by the German Federal Statistical Office (Statistisches Bundesamt) - that total migration over all groups is 200,000 annually and then distribute this total migration to the three groups ho, rw, as according to the relative shares during the years 2008-2012.

\footnotetext{
${ }^{38}$ The advantage of constructing the flow data from the information on the population stock is that we can meaningfully measure the net addition to the stock caused by migration. Also, direct information on flows features statistical inaccuracies because of double counting of multiple within year migration. The disadvantage is that we do have to make assumptions on mortality and survival rates for all population groups. However, mortality is relevant only at higher ages at which migration numbers are close to zero.
} 
2. Age and group specific fertility distributions are constant at their respective age specific averages taken over the years 2007-2018 until year 2100. Thereafter, fertility rates adjust such that the number of newborns is constant in each period. With this assumption (and with the assumption of constant survival rates and constant migration numbers) the population will reach a stationary distribution with constant population growth by about year 2200 .

3. Survival rates increase according to the predictions from the Lee-Carter model until year 2100 and are constant thereafter.

During the phase-in period from 1960 to 2012 we have the exact data on the population stocks only from 2008 onwards. Leading towards 2008 we forward shoot on the population dynamics using data on the annual flow of migration and distribute those across the four groups such that we minimize the distance between the model implied population stocks in the four groups in 2008 and the respective actual population stock.

The next subsections contain a more detailed description of the construction of fertility rates, mortality rates and migration numbers.

\section{C.1.1 Fertility Rates}

In the data the number of newborns is

$$
N_{t+1}(0, i)=\sum_{j=j_{f}}^{j_{c}} f_{t}(j, i) N_{t}(j, i, f e)
$$

where $f_{t} j, i$ is the group $i$ age $j$ and time $t$ specific fertility rate. Since we lack information on $f_{t}(j, i)$ and on the number of newborns for all population groups, we construct fertility rate as follows. We take time and age specific fertility rates of the average German population from the Federal Statistical Office and on the number of birth from the Human Mortality Database, separately for East and West Germany. Based on the stock of the population in both regions, we next adjust the age- and time-specific fertility rates such that the fertility distribution is consistent with the number of newborns. We then take the population weighted average of the East and West German constructed data.

\section{C.1.2 Mortality Rates}

We take a time series of gender specific mortality rates for 1950 to 2017 from the Human Mortality Database, computed as the weighted average of East and West German mortality 
rates, and decompose mortality rates as

$$
\ln \left(1-\psi_{t}(j, g)\right)=a(j, g)+b(j, g) d_{t}(g)
$$

where $\psi_{t}(j, g)$ is the survival rate applying the Lee-Carter procedure (Lee and Carter 1992). Next, we assume that the estimated time specific factor $\hat{d}_{t}(g)$ obeys a unit root process

$$
\hat{d}_{t+1}(g)=\alpha(g)+\hat{d}_{t}(g)+\epsilon_{t+1}(g) .
$$

Based on the estimates $\{\hat{a}(j, g), \hat{b}(j, g)\}_{j=0}^{J}, \hat{d}_{t}(g), \hat{\alpha}(g)$ we predict (future) survival rates by setting to zero the innovation terms $\hat{\epsilon}_{t}(g)$ and initialize the process assuming that $\hat{\hat{d}}_{0}(g)=$ $\hat{d}_{0}(g)$.

After construction of the population numbers (and the migration flows, see next subsection) we take population weighted average survival rates and recompute the population dynamics.

\section{C.1.3 Migration Numbers}

We construct the net addition to the respective population stock in group $i$ by backing out the net flow from equation (29): ${ }^{39}$ Since we lack data on group specific mortality rates, we assume that all immigrants immediately after entry have the same mortality process as the average German population and thus set $\psi_{t}(j, i, g)=\psi_{t}(j, g) \forall i$.

Figure 13 summarizes the constructed migration flows in the three groups of the foreign population $\{h o, r w, a s\}$, Figure 14 contains the according age distribution of the migration flow, and Figure 15 shows the resulting age distribution of the population in groups $i \in$ $\{n a, h o, r w, a s\}$.

\section{C.2 Age Wage Profiles}

Figure 16 displays the age wage profiles for natives and for foreigners from group rw. Age wage profiles for foreigners from groups ho are similar to those of natives.

\footnotetext{
${ }^{39}$ In the data, the population stock is reported at the end of a given calendar year which we accordingly interpret as the beginning of the next calendar year. Thus the population stock reported in the data at the end of calendar year 2007 is taken to be the population stock at the beginning of year 2008 .
} 
Figure 13: Net Migration Flows

(a) Group ho

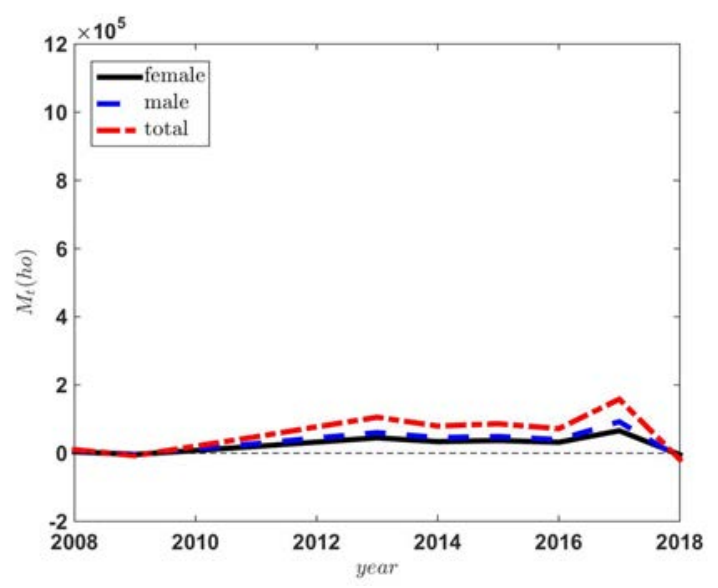

(c) Group as

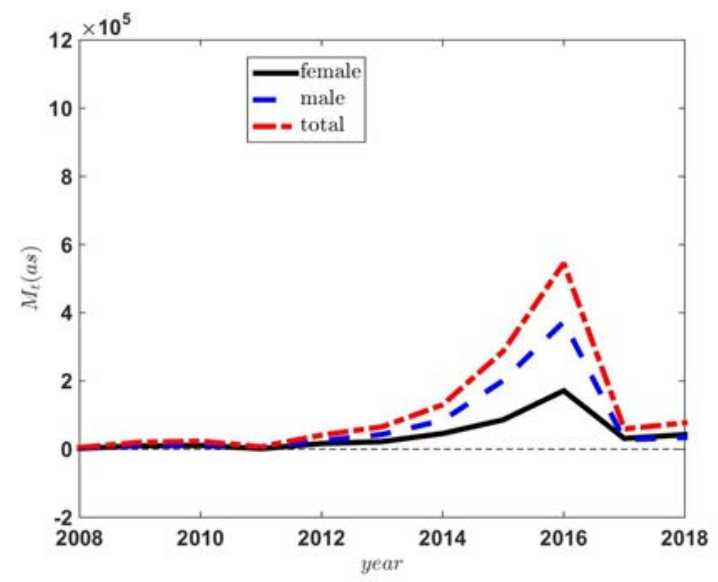

(b) Group rw

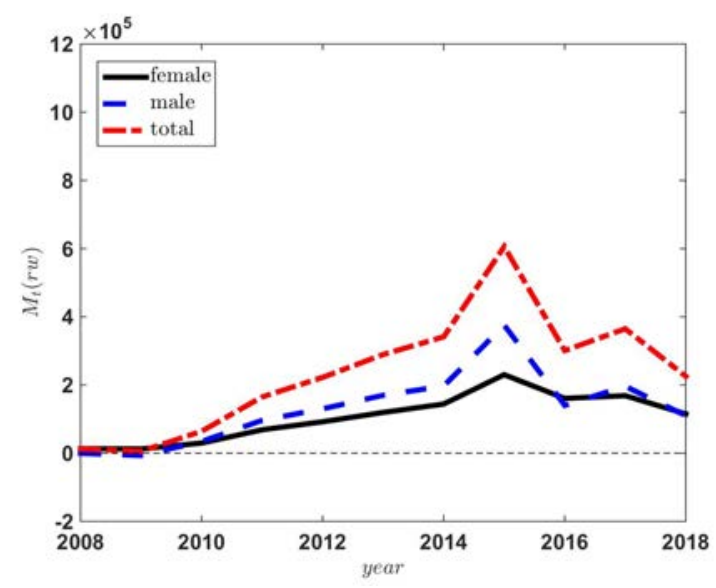

(d) Total

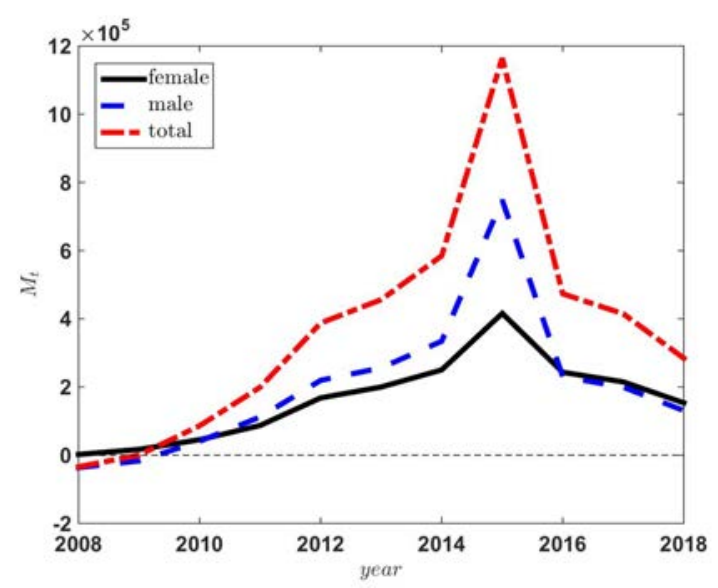

Notes: Annual aggregate net migration numbers from 2008 to 2018 by nationality group. Panel (a): HIOECD, Panel (b): RW, Panel (c): AS, Panel (d): total. Source: Own calculations based on Central Foreign Population Registry (Ausländerzentralstatistik, AZR). 
Figure 14: Age Distribution of Net Migration

(a) Group ho

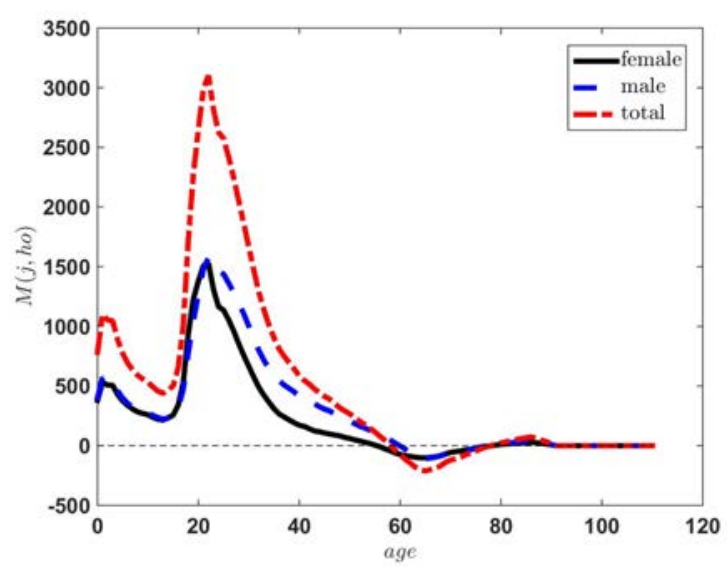

(c) Group as

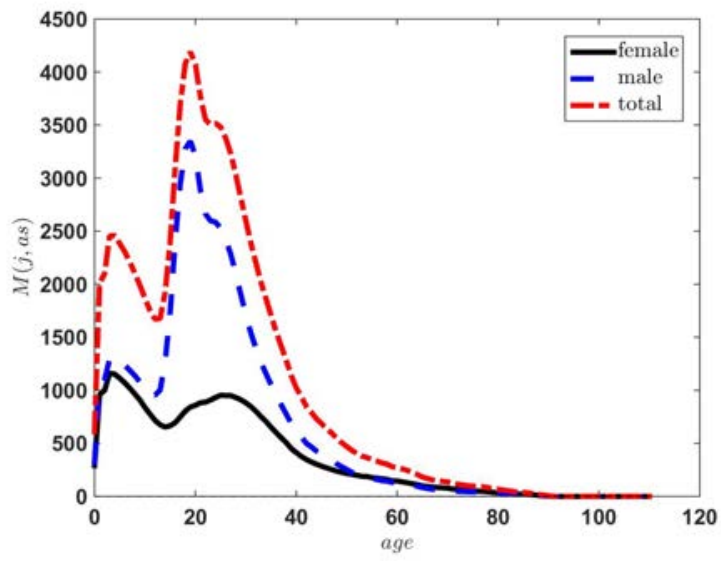

(b) Group $r w$

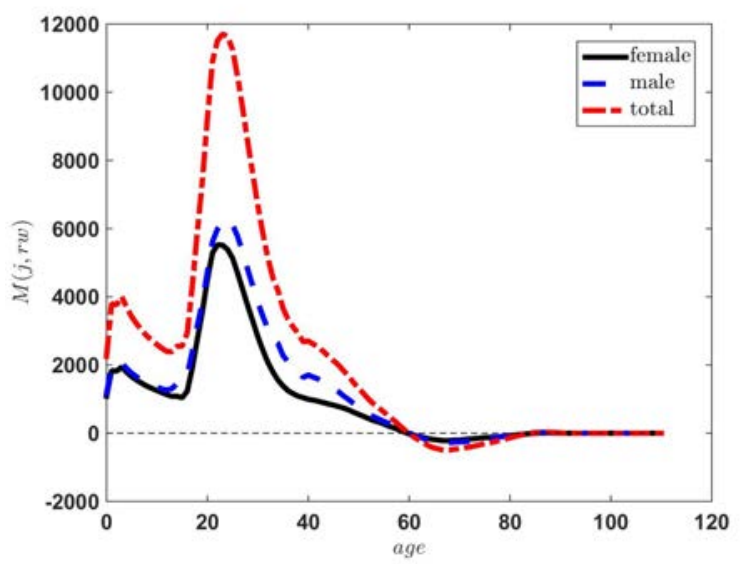

(d) Total

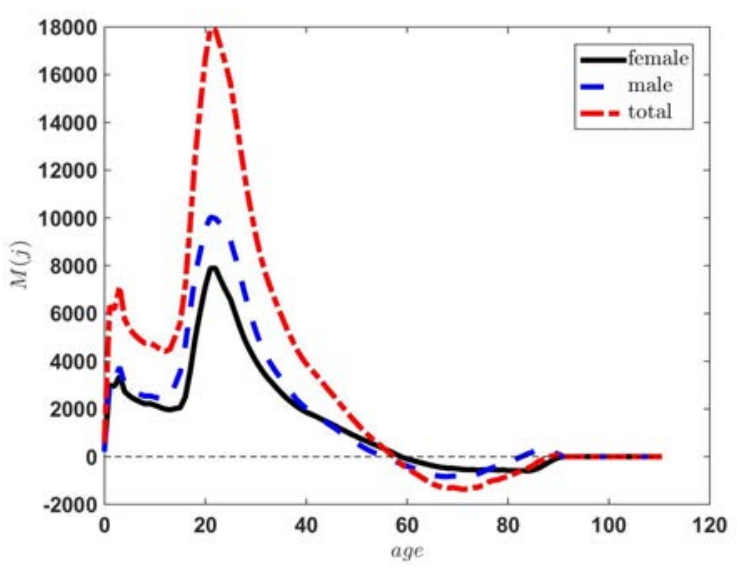

Notes: Age distribution of net migration, average of years 2008 to 2018. Panel (a): HIOECD, Panel (b): RW,

Panel (c): AS, Panel (d): total. Source: Own calculations based on Central Foreign Population Registry (Ausländerzentralstatistik, AZR). 
Figure 15: Age Distribution of Population Stock

(a) Group na

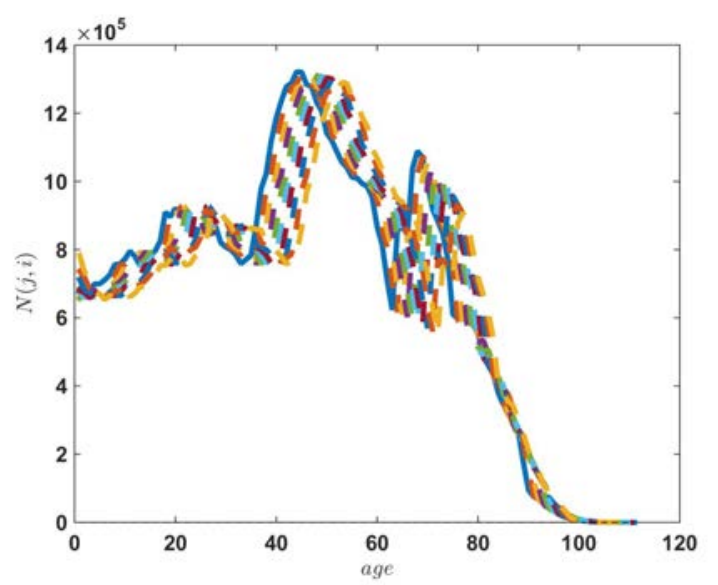

(c) Group rw

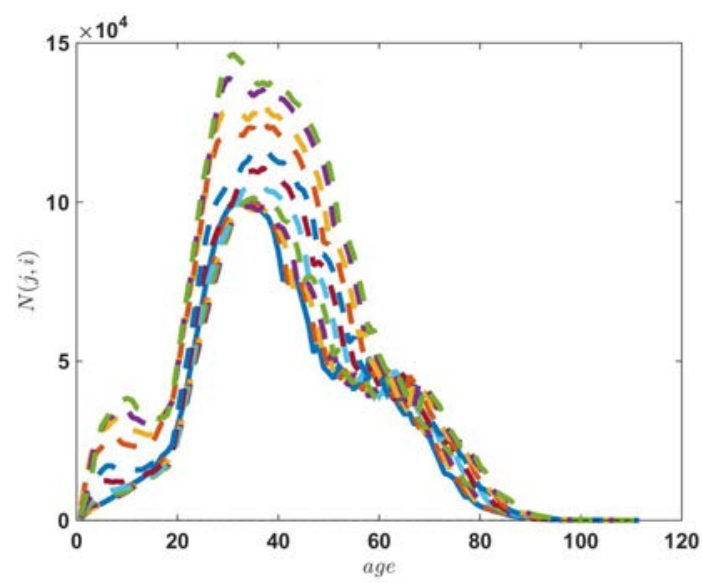

(b) Group ho

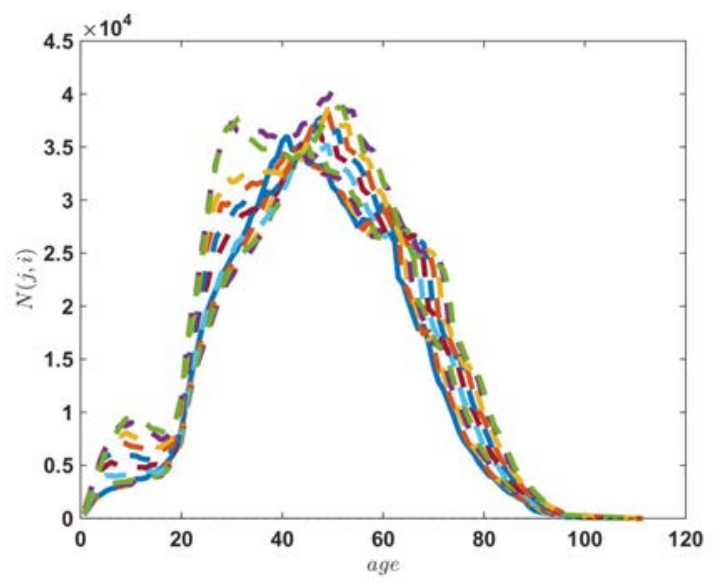

(d) Group as

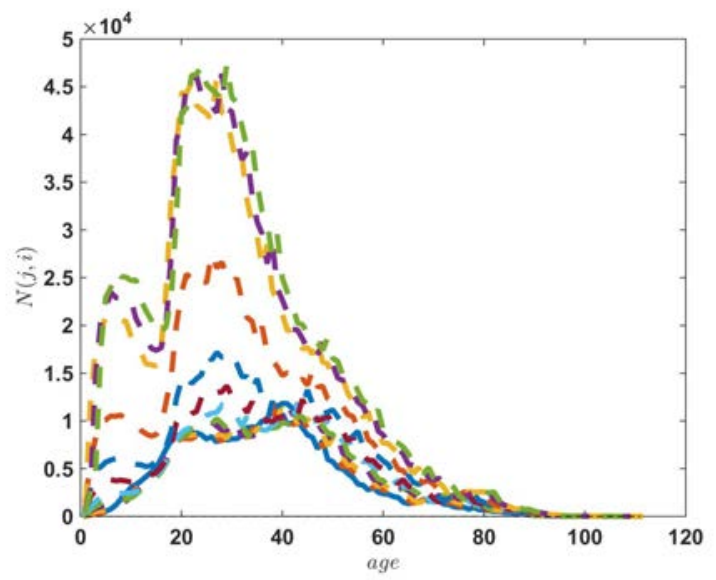

Notes: Age distribution of population stock by years 2008 to 2018. Panel (a): Natives, Panel (b): HIOECD, Panel (c): RW, Panel (d): AS. Source: Own calculations based on Central Foreign Population Registry (Ausländerzentralstatistik, AZR) and German Federal Statistical Office (Statistisches Bundesamt). 
Figure 16: Age Wage Profiles

(a) Natives

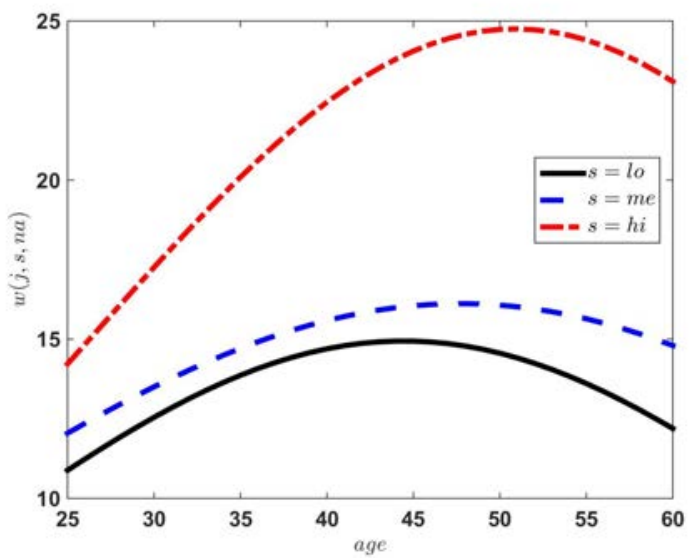

(b) Foreigners from group $r w$

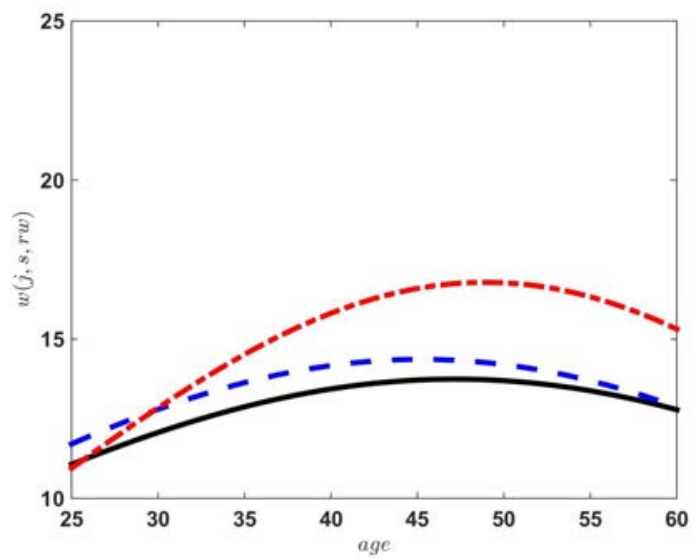

Notes: Predicted age wage profiles for natives and foreigners from RW for the three skill categories $s \in$ $\{l o, m e, h i\}$. Source: Own calculations based on SOEP. 


\section{C.3 Technology}

For the estimation of the substitution elasticities in production, we exploit the homogeneity of the production function in each nest and add productivity parameters $\tilde{\epsilon}(\cdot)$ at each nest, which are normalized to one. Thus, at the estimation, we write labor at each nest as

$$
\begin{aligned}
L_{t} & =\left(\sum_{s \in\{l o, m e, h i\}} \tilde{\epsilon}(s) \tilde{L}_{t}(s)^{1-\frac{1}{\sigma_{l m h}}}\right)^{\frac{1}{1-\frac{1}{\sigma_{l m h}}}} \\
\tilde{L}_{t}(s) & =\sum_{\bar{j}=1}^{n_{\bar{j}}} \tilde{\epsilon}(\bar{j}, s) \tilde{L}_{t}(\bar{j}, s) \\
\tilde{L}_{t}(\bar{j}, s) & =\left(\tilde{\epsilon}(\bar{j}, s, n a) \tilde{L}_{t}(\bar{j}, s, n a)^{1-\frac{1}{\sigma_{n f}}}+\tilde{\tilde{\epsilon}}(\bar{j}, s, f o) \tilde{\tilde{L}}_{t}(\bar{j}, s, f o)^{1-\frac{1}{\sigma_{n f}}}\right)^{\frac{1}{1-\frac{1}{\sigma_{n f}}}} \\
\tilde{\tilde{L}}_{t}(\bar{j}, s, f o) & =\left(\tilde{\epsilon}_{\tilde{\epsilon}}(\bar{j}, s, h o) \tilde{L}_{t}(\bar{j}, s, h o)^{1-\frac{1}{\sigma_{h r}}}+\left(\sum_{i \in\{r w, a s\}} \tilde{\epsilon}(\bar{j}, s, i) \tilde{L}_{t}(\bar{j}, s, i)\right)^{1-\frac{1}{\sigma_{h r}}}\right)^{\frac{1}{1-\frac{1}{\sigma_{h r}}}} \\
\tilde{L}_{t}(\bar{j}, s, i) & =\sum_{g \in\{f e, m a\}} \tilde{\epsilon}(\bar{j}, s, i, g) L_{t}(\bar{j}, s, i, g),
\end{aligned}
$$

and we assume that $\tilde{\epsilon}(\bar{j}, s, i, g)=\tilde{\epsilon}(\bar{j}, s, i) \tilde{\epsilon}(g)$. Given the homogoeneity of the production function, it is straightforward to show that the productivity scaling parameters $\tilde{\epsilon}(\cdot)$ can be mapped into labor productivity $\epsilon(\bar{j}, s, i) \epsilon(g)$, where $\epsilon(g)=\tilde{\epsilon}(g)$, and $\epsilon(\bar{j}, s, i)$ is an average over $j \in\left[j_{l}(\bar{j}, s), \ldots, j_{h}(\bar{j}, s)\right]$ of the $(s, i)$-specific productivity profile $\epsilon(j, s, i)$.

We estimate the elastiticities of substitution at the different nests jointly with the productivity parameters following the standard approach in the literature (cf., e.g., Borjas 2003). For example, at the level of immigrant groups, we translate the first order conditions into estimation equations and identify the relative productivity parameters and the elasticity of substitution across immigrant groups using variation over time in the relative labor supply (hours worked) and the relative wages. We then use these estimates to obtain the CES aggregator of labor supply of immigrants, and the implied wage aggregate. We use this together with labor supply and wages of natives to estimate the next layer of the CES, and then move up nest by nest in the same fashion. At each nest, we use the population size of a given group as an instrument for the labor supply in order to address a potential endogeneity problem of the estimation equations. At the highest nest, we allow the education group specific productivity components to follow quadratic time trends in order to accomodate the possibility of skill-biased technological change in the estimation. We implement the estimator using SOEP data from 1984-2015. For each year, group specific hours worked are 
aggregate hours worked by individuals up to age 60 of a given group, and the group specific wage rate is estimated using workers up to age 60 who work at least 520 hours.

\section{C.4 Social Insurance}

Figure 17 shows the contribution rates to the German PAYG pension system and to the public health insurance system (including long-term care insurance).

Figure 17: Contribution Rates to Social Security \& Health Insurance

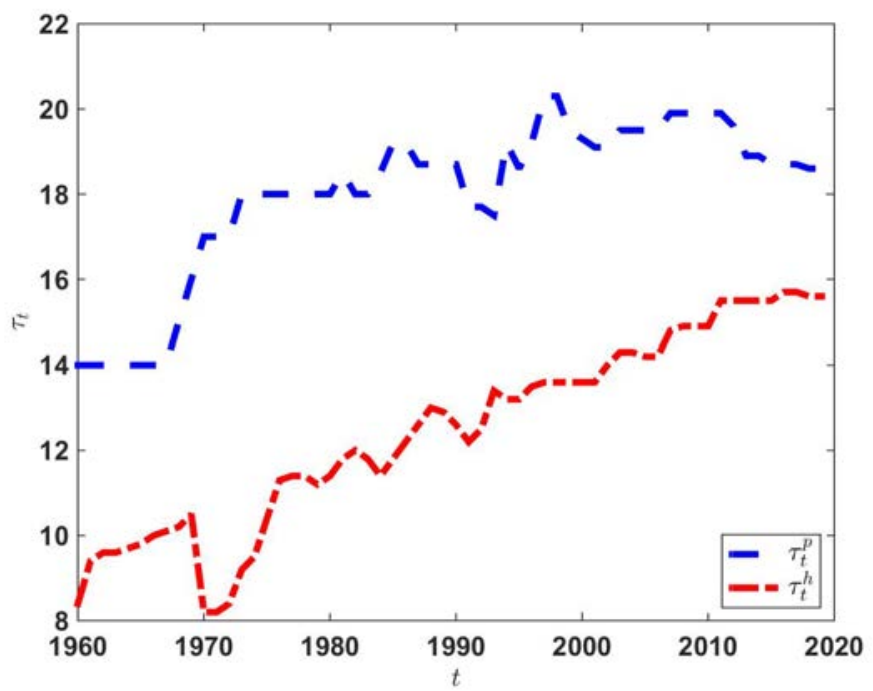

Notes: Data on contribution rates to social security and health insurance. Source: http://www. sozialpolitik-aktuell.de.

Our data on health expenditures cover ages 0-99 for years 2010-2017. We normalize these expenditures by nominal GDP data (which leads to almost identical profiles for all years pointing to strong time effects) and take the average across these years. Figure 18 shows the age profile for females and males.

\section{Appendix: Further Results}

\section{D.1 Population Shares by Groups}

Figures 19 and 20 shows the population shares by nationality and their changes relative to the baseline scenario. 
Figure 18: Health Expenditures over the Life-Cycle [Index, centralized data]

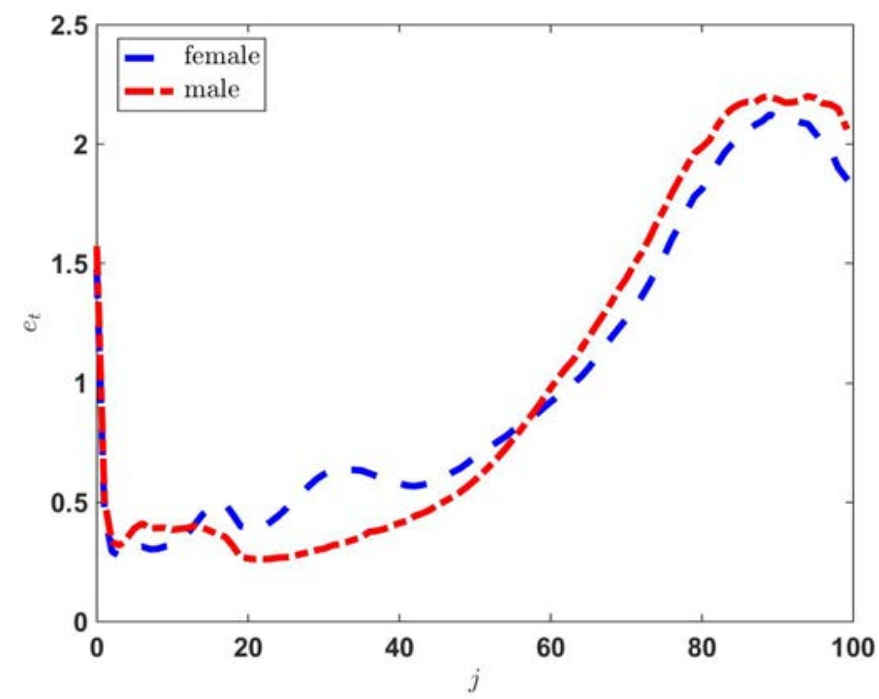

Notes: Data on age-specific health expenditures. Source: Federal Insurance Office (Bundesversicherungsamt).

\section{D.2 The Fiscal Side}

Figure 21 shows the health contribution rate (and its change). In the refugee migration scenario the health contribution rate increases slightly; refugee immigrants receive the same age contingent lump-sum payments but contribute little to the system. In the high migration scenario the contribution rate initially increases when the effect of young in-migration dominates.

Figure 22 shows total government expenditures as the sum of government consumption $G_{t}$ and all outlays to finance incoming and leaving refugees $E_{t}$. In the migration scenarios we observe the initial blip due to the incoming wave of migrants, but overtime overall expenditures decrease slightly relative to GDP because GDP increases.

\section{D.3 Per Capita GDP and Consumption}

Figure 23 de-trended per capita GDP and consumption, where de-trending is by the technology level $A_{t}$. 
Figure 19: Population Shares by Region of Origin I

(a) Group na

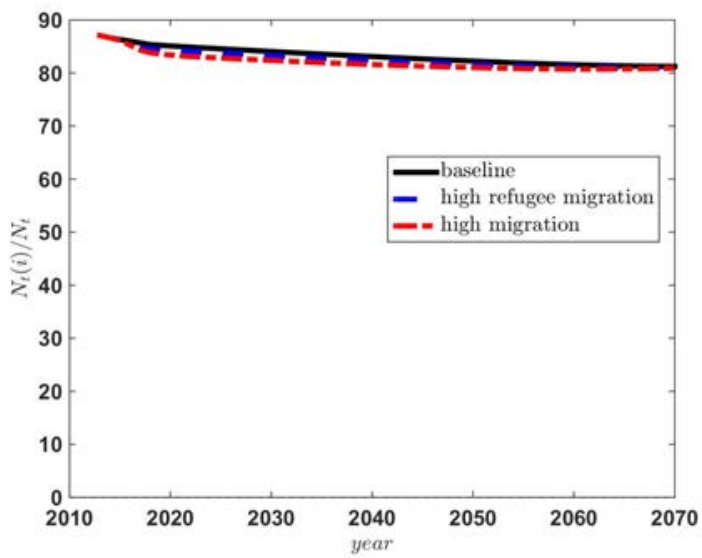

(c) Group ho

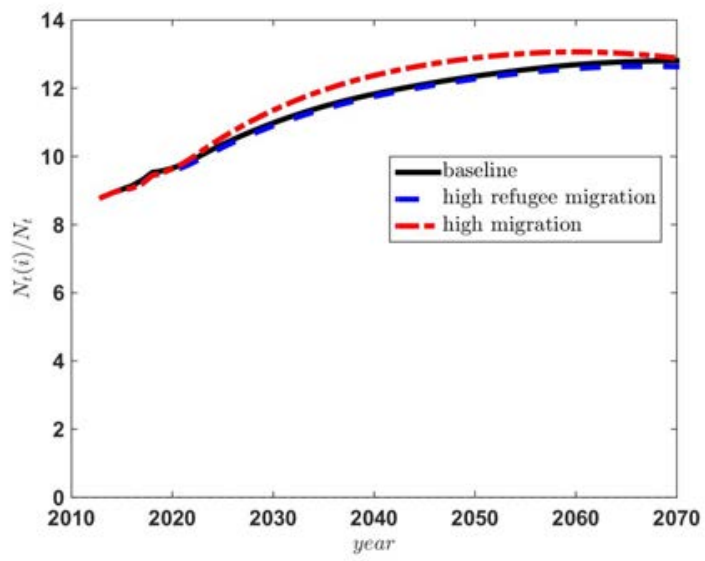

(b) \%-Change roup na

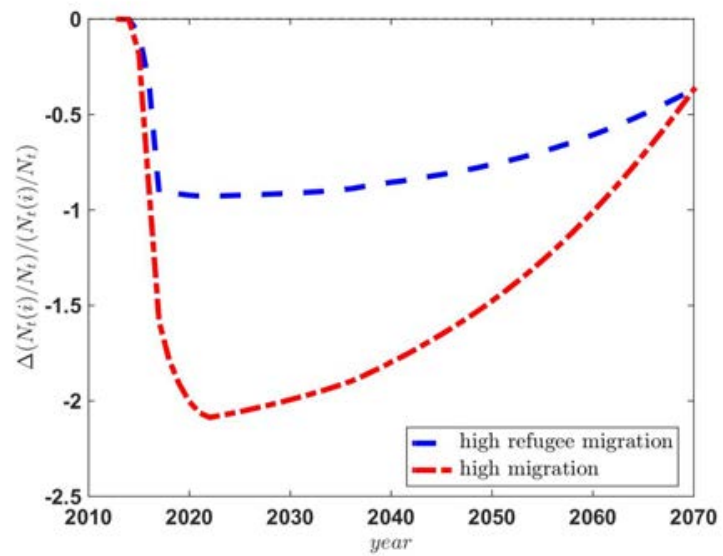

(d) \%-Change group ho

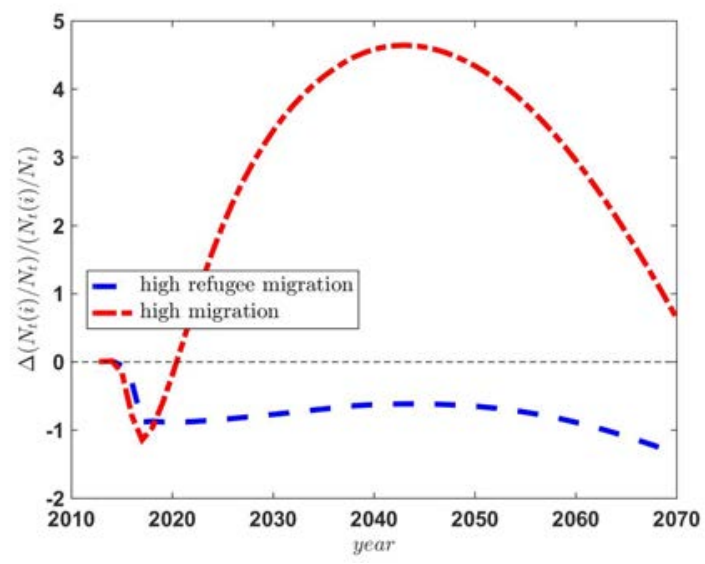

Notes: Fractions as a share of total population and respective percentage changes. Panels (a)-(b): Natives, Panels (c)-(d): HIOECD.

\section{D.4 Rate of Return \& Wages}

Figure 24 shows the rate of return to capital and its change to the baseline demographic model. Figure 25 shows gross and net wages of low skilled natives as weighted averages of the three age groups.

\section{D.5 Wage Changes}

Figure 26 displays the change of the skill ratios for the low-skilled natives. 
Figure 20: Population Shares by Region of Origin II

(a) Group $r w$

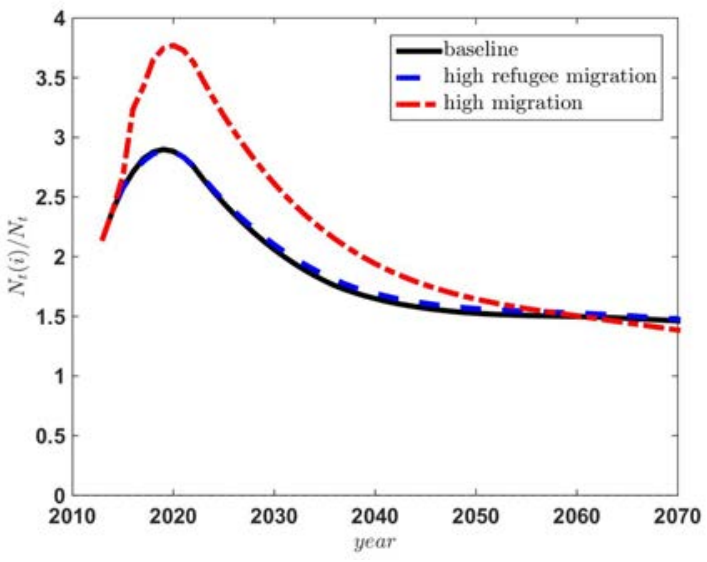

(c) Group as

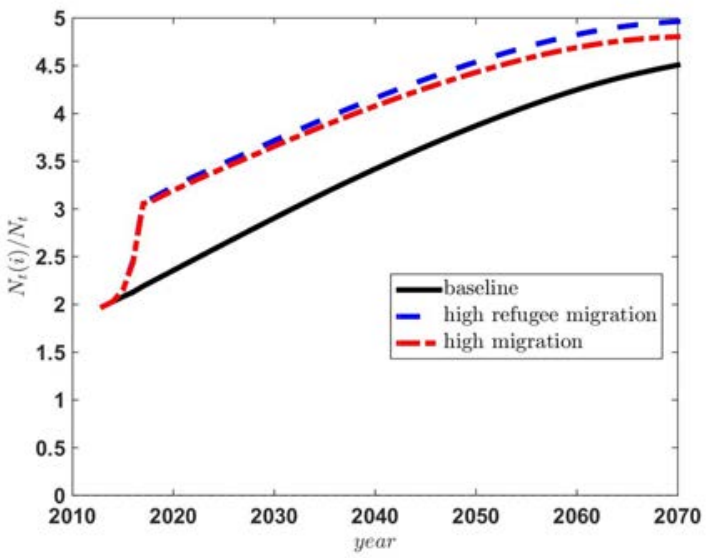

(b) \%-Change group $r w$

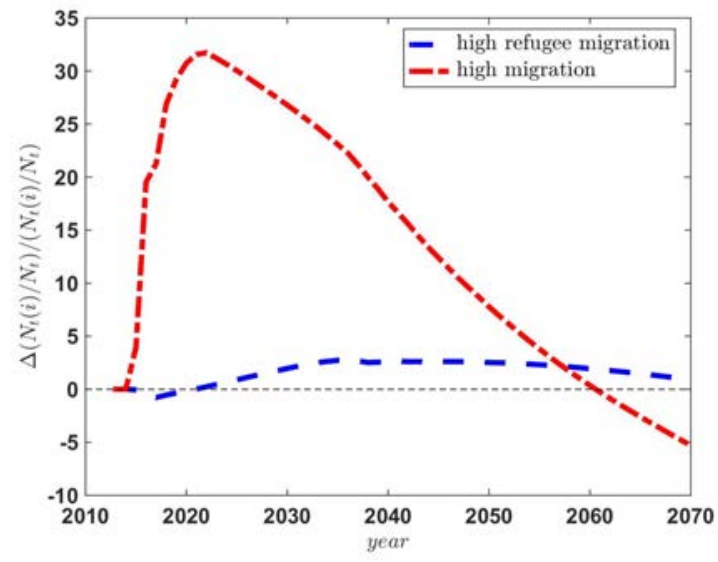

(d) \%-Change group as

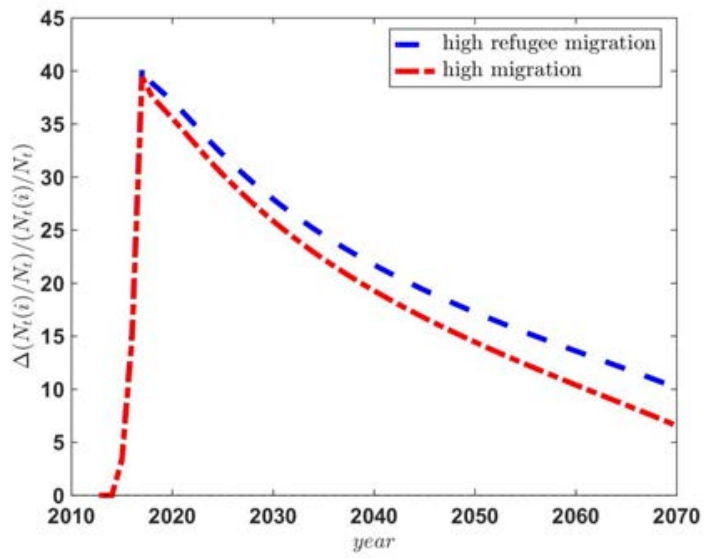

Notes: Fractions as a share of total population and respective percentage changes. Panels (a)-(b): RW, Panels (c)-(d): AS. 
Figure 21: Health Contribution Rate

(a) Health Contr. Rate $\left(\tau_{t}^{h}\right)$

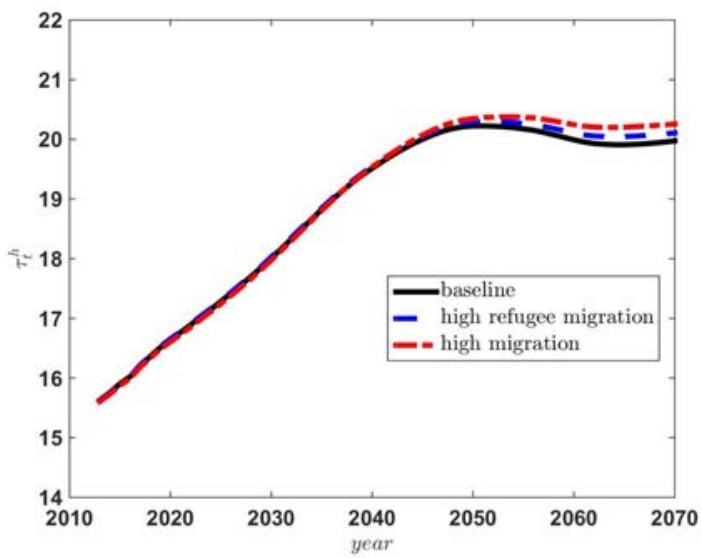

(b) \%p-Change of Total Health Contr. Rate

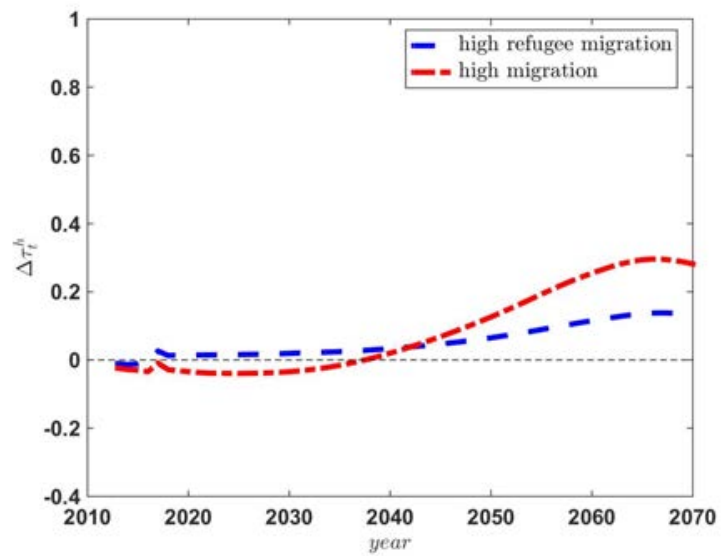

Notes: Panel (a): contribution rate to health insurance system, Panel (b): percentage point change of contribution rate to health insurance system.

Figure 22: Total Government Expenditures
(a) Total Gov. Exp. to GDP $\left(G_{t}+E_{t}\right) / Y_{t}$
(b) \%p-Change of Total Gov. Exp. to GDP
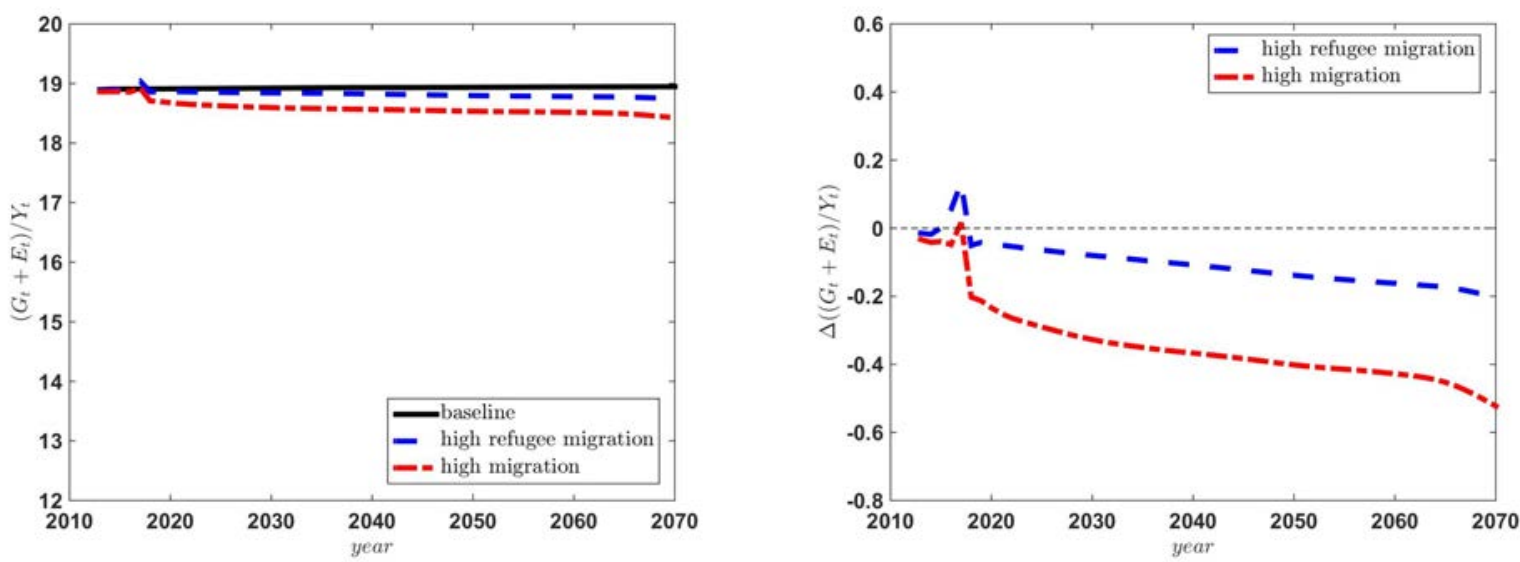

Notes: Panel (a): ratio of total government expenditures to GDP, Panel (b): percentage point change of ratio of total government expenditures to GDP. 
Figure 23: Detrended Per Capita GDP \& Consumption [Index]

(a) Per Capita GDP

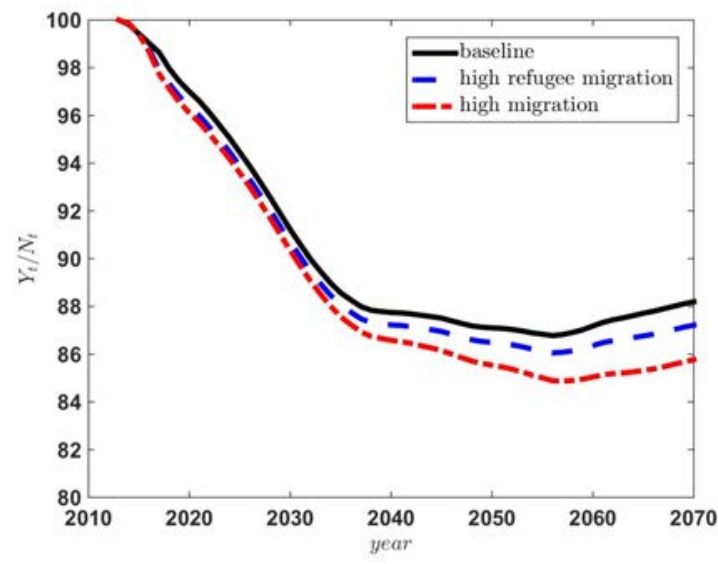

(c) Per Capita Consumption

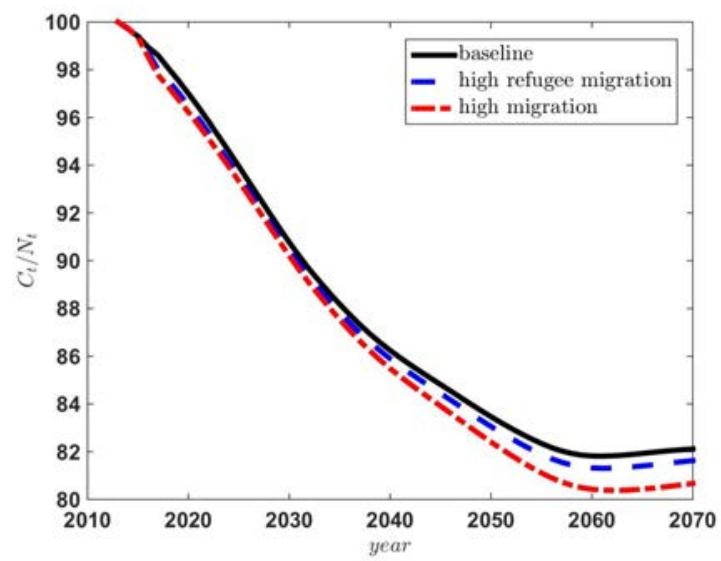

(b) \%-Change of P.C.GDP

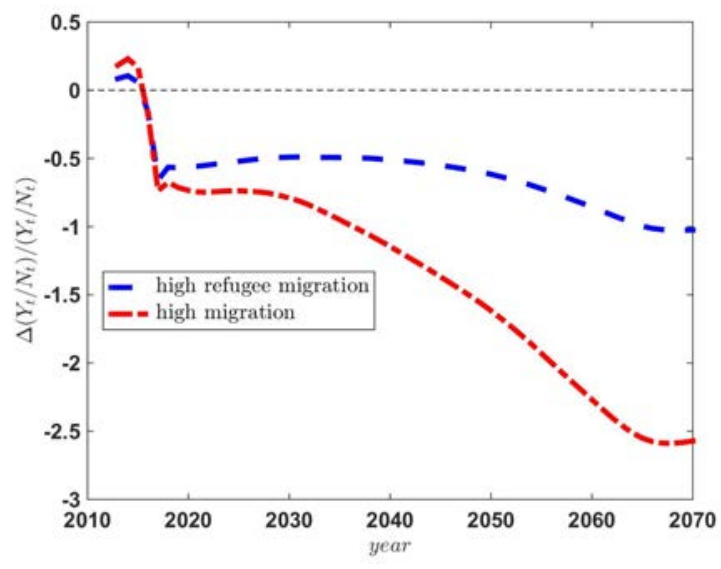

(d) \%-Change of P.C.Consumption

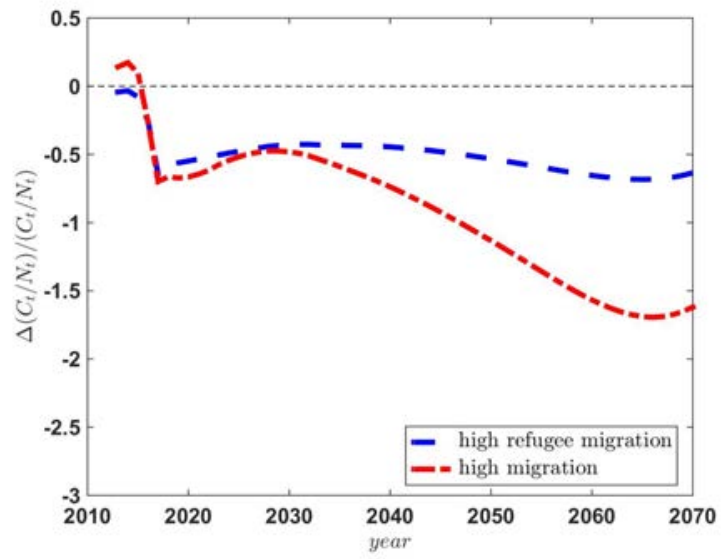

Notes: Panel (a): de-trended per capita GDP (Index, 2013=100), Panel (b): percent change of per capita GDP; Panel (c): de-trended per capita consumption (Index, 2013=100), Panel (d): percent change of per capita consumption. 
Figure 24: Rate of Return

(a) Rate of Return

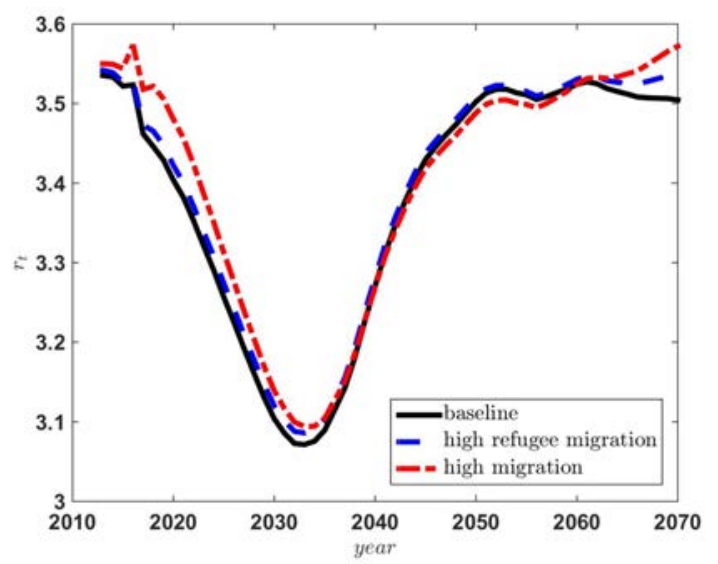

(b) \%p-Change of Rate of Return

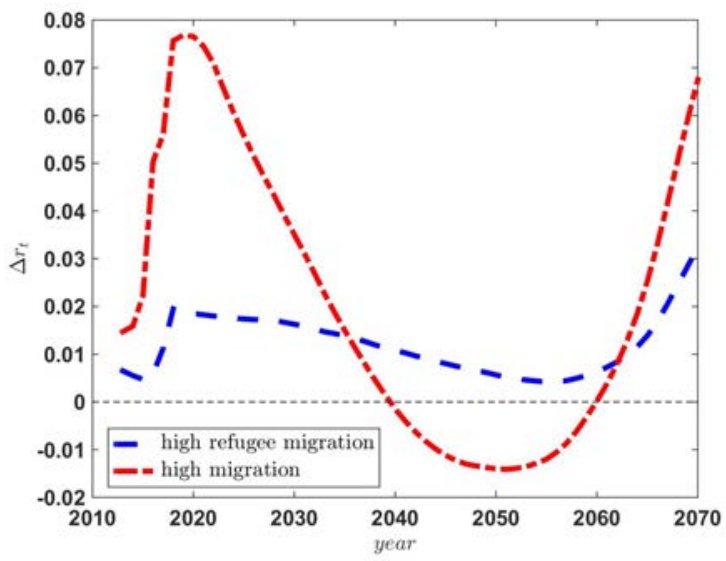

Notes: Panel (a): rate of return, Panel (b): percentage point change of rate of return.

Figure 25: Gross \& Net Wages, Low-Skilled Natives, Age-Group $\bar{j}=1$

(a) Gross Wages

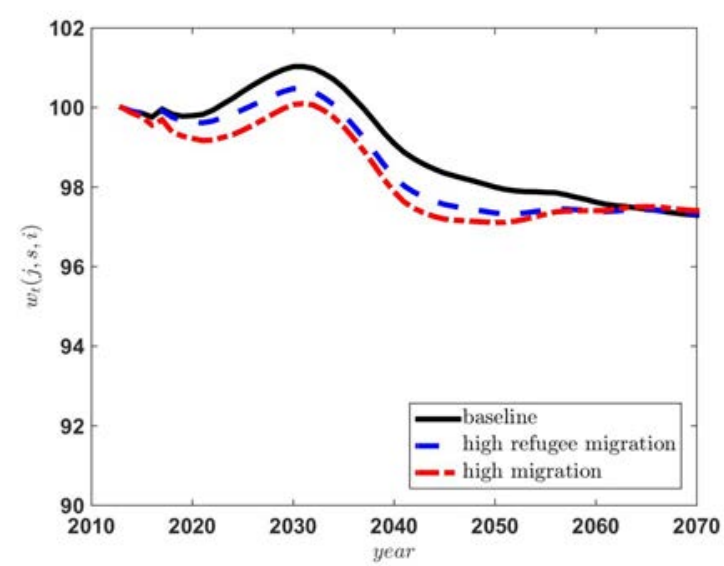

(b) Net Wages

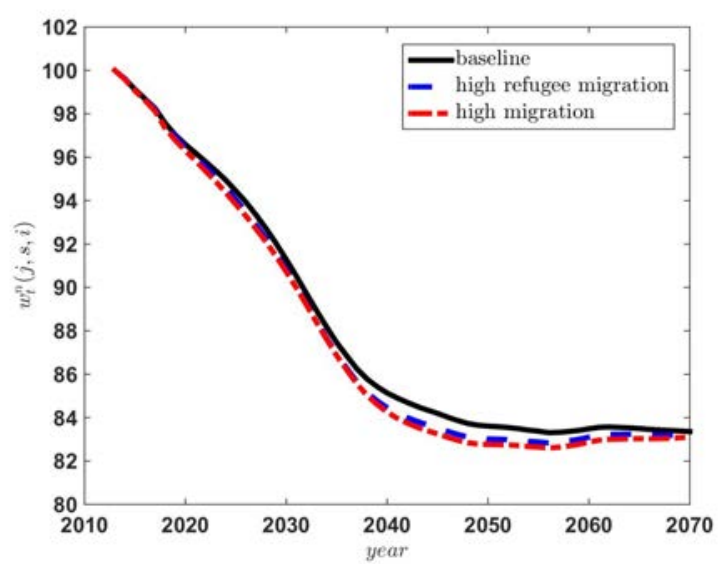

Notes: Panel (a): gross wages, Panel (b): net wages of low skilled natives in age group $\bar{j}=1$. 
Figure 26: Change of Skill Ratios, Low-Skilled Natives, Age Group $\bar{j}=1$
(a) $\Delta \Theta_{t}(m e, l o)$
(b) $\Delta \Theta_{t}(h i, l o)$
(c) $\Delta \Theta_{t}(f o, n a \mid l o)$
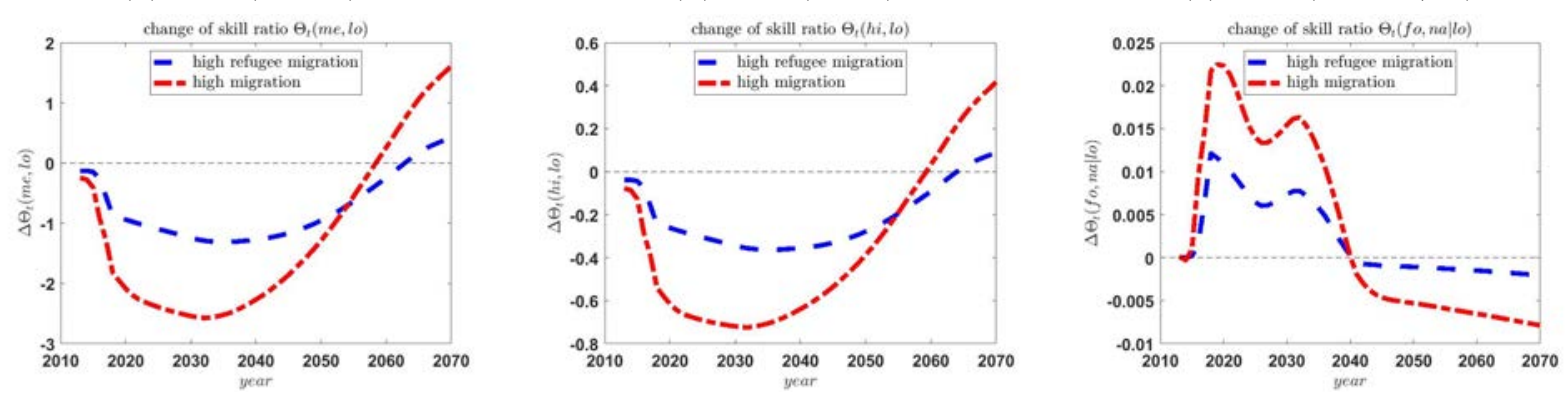

Notes: Change of skill ratios as defined in equation (46) for low skilled natives in age group $\bar{j}=1$. 